\title{
The Normal Value of Electrocardiogram in the Japanese
}

\author{
The Report of the Committee on the Criteria \\ of the Heart Disease and Electrocardiogram
}

\begin{abstract}
This survey was made by the members of the Committee on the Criteria of the Heart Disease and Electrocardiogram sponsored by the Ministry of Education in Japan. The members of the Committee who participated in this survey were as follows.

Chairman: H. Ueda, Professor, University of Tokyo, Tokyo

Members : I. Donomae, Professor, University of Osaka, Osaka

E. Kimura, Professor, Nippon Medical School, Tokyo

N. Kimura, Professor, Kurume University, Kurume

M. Maekawa, Professor, University of Kyoto, Kyoto

H. Niitani, Associate Professor, Showa Medical College, Tokyo

S. Okinaka, Professor, University of Tokyo, Tokyo

M. Saito, Associate Professor, University of Tokyo, Tokyo

T. Sano, Associate Professor, Tokyo Medical and Dental University, Tokyo

S. Tasaka, Professor, University of Tokyo, Tokyo

T. Takatsu, Professor, University of Tokyo, Tokyo

M. Takayasu, Professor, Mie Prefectural University, Tsu
\end{abstract}

A few reports have been conducted to describe the normal electro-
cardiogram of Japanese people. Their results were based on a relatively so small and limited population that, for enhancing the utility of the electrocardiogram in clinical practice, it is needed to detect abnormal electrocardiogram from the normal by delineating the range of normal variations of electrocardiographic component parts, which are based on the large samples assembled from all over Japan.

In 1959 the Committee on the Criteria of the Heart Diseases and Electrocardiogram was established under the chairman of Hideo Ueda with the support of the Ministry of Education. The objects of the committee were :

(a) to determine the normal values of various electrocardiographic component parts for each age group in Japanese people

(b) to study the prognosis of the patients with abnormal electrocardiographic findings 
(c) to estimate the clinical value of vectorcardiography

(d) to determine the electrocardiographic criteria of coronary heart disease

(e) to conduct the comparative study of electrocardiogram and pathological findings

The committee conducted a survey for 2 years, and various problems were analyzed on the basis of the data from the members of the committee. The purpose of this report is to provide the normal value of electrocardiographic component parts in Japanese people.

\section{METHOD}

Electrocardiographic records of 1,329 normal persons are assembled from institutes of the members of the committee. The persons with following conditions are excluded as abnormal.

(a) persons with blood pressure over $140 \mathrm{~mm} . \mathrm{Hg}$ in systolic and $90 \mathrm{~mm} . \mathrm{Hg}$ in diastolic

(b) patients restricted to bed

(c) patients with diabetes mcllitus

(d) patients with tuberculosis being treated

(e) persons with albuminuria

(f) patients with cardiac diseases

(g) persons with abnormal findings in chest $\mathrm{X}$-ray film

(h) patients with anemia

(i) patients with pulmonary emphysema

(j) patients with beriberi

The measurement of electrocardiograms, the kind of the electrocardiograph and the technique to take the electrocardiogram were entrusted to the each committee member, and tabulated results were sent to the central committee where mathematical calculations were performed. The standard deviation (SD) is calculated on some components, of which population samples show normal distribution or near it. A range of $2 \mathrm{SD}$ in either direction of the mean is set as the normal range.

The electrocardiographic component parts submitted to analysis are the voltages of $\mathrm{P}, \mathrm{Q}, \mathrm{R}, \mathrm{S}, \mathrm{T}$ and $\mathrm{U}$ deflections, position of $\mathrm{S}-\mathrm{T}$ segment, intervals of $\mathrm{P}-\mathrm{Q}$, durations of $\mathrm{P}, \mathrm{QRS}$ and $\mathrm{Q}$. Ventricular activation time is measured in lead $V_{1}, V_{2}, V_{5}$ and $V_{6}$. Voltages are measured in standard leads, unipolar leads of extremity $\left(V_{R}, V_{L}, V_{F}\right)$ and precordium $\left(V_{4 R}, V_{1}, V_{2}, V_{3}, V_{4}, V_{5}\right.$ and $\left.V_{6}\right)$. The intervals and durations are measured in one arbitrarily selected lead of standard leads and precordial leads.

\section{Results}

The age groups and the number of subjects are listed in Table I.

The age groups are divided into 2 parts by sex. 
Table I. Number of Records Analyzed in Each Age Group

\begin{tabular}{lcccccccc}
\hline \multicolumn{8}{c}{ Age Group (yr.) } \\
\hline Age & $15 \sim$ & $20 \sim$ & $30 \sim$ & $40 \sim$ & $50 \sim$ & $60 \sim$ & $70 \sim$ & Total \\
\hline Male & 61 & 165 & 119 & 153 & 135 & 101 & 48 & 782 \\
\hline Female & 39 & 109 & 94 & 82 & 102 & 79 & 42 & 547 \\
\hline Total & 100 & 274 & 213 & 235 & $\frac{237}{13}$ & 180 & 90 & 1329
\end{tabular}

\section{$P$ Deflection}

\section{(1) Duration of $\mathrm{P}$}

The values of $\mathrm{P}$ deflection are given in Table II.

In standard and unipolar extremity leads, the mean values are from $0.09 \mathrm{sec}$. to $0.10 \mathrm{sec}$. among age groups, and there are no apparent variations with ages. In each group, the standard deviation is almost the same and the calculated normal values of $P$ deflection range from 0.13 sec. to $0.06 \mathrm{sec}$.

In precordial leads, the mean values are from $0.08 \mathrm{sec}$. to $0.10 \mathrm{sec}$, which are a little shorter compared with those of standard leads.

\section{(2) Voltage of $\mathrm{P}$ deflection (Table III)}

As for the amplitude of $\mathrm{P}$, there are no remarkable differences with sex and age. The greatest amplitude of $\mathrm{P}$ is shown in lead II, and normal limit of it ranges from $1.88 \mathrm{~mm}$. to $0.19 \mathrm{~mm}$. All cases are below $2.6 \mathrm{~mm}$. in lead II. In lead III, lowest limit indicates below zero line in all cases, but does not exceed $-0.5 \mathrm{~mm}$.

\section{$P-Q$ Interval}

P-Q interval is mcasured in each one lead of extremity leads and S D in each group is given in Table IV. Most cases have the mean value of $0.16 \mathrm{sec}$. and the maximum of them is $0.17 \mathrm{sec}$. in 60 year male group, and the minimum is $0.16 \mathrm{sec}$. in 20 year female group. Normal range is $0.23 \mathrm{sec}$. to $0.10 \mathrm{sec}$. and the minimum valuc of most cases is over $0.11 \mathrm{sec}$. The precordial leads are almost the same as the extremity leads.

\section{QRS Interval}

The QRS interval was determined in the lead which demonstrated the largest value in each extremity and precordial lead as in the duration of $\mathrm{P}$. The mean values range from $0.07 \mathrm{sec}$. to $0.09 \mathrm{sec}$. The normal upper limit is $0.11 \mathrm{sec}$. There are no remarkable differences in mean values with age and sex. The mean values of QRS interval of precordial 
leads are almost the same or a little longer compared with of standard leads.

\section{Q Deflection}

The duration and voltage of $Q$ deflection are determined and averaged in standard leads, unipolar leads and precordial leads, in cases of each group in which they are measurable. $Q$ duration is measured in 2 components, that is, $Q_{1}$ which is the time from the beginning of the QRS complex to the bottom of $Q$ deflection, and $Q$ Ir, which is the duration from the beginning of the QRS to the point where $Q$ deflection crosses the base line.

\section{(1) Voltage of $Q$ deflection (Table VI)}

In all standard leads and unipolar extremity leads, the mean values are smaller than $1 \mathrm{~mm}$. except ${ }_{a} V_{R}$. There are no remarkable differences with sex, but some tendency to decrease with age in lead II, III and ${ }_{a} V_{F}$ and to increase in ${ }_{a} V_{L}$ in the mean value of $Q$ deflection.

Very few cases have small $Q$ deflection previous to $R$ deflection in the leads $V_{1}, V_{2}$ and $V_{3}$, which are also under calculation. The mean values of $Q$ in leads $V_{5}$ and $V_{6}$ are very small, which range from 0.04 $\mathrm{mm}$. to $1.10 \mathrm{~mm}$. Maximum values do not exceed $5.2 \mathrm{~mm}$. There are no remarkable difference in age and sex.

\section{(2) Duration of $Q_{I}$ (Table VII)}

In standard leads and unipolar extremity leads, the mean values

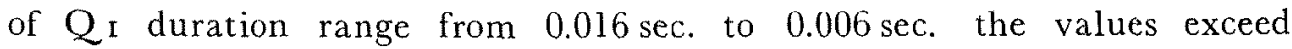
$0.010 \mathrm{sec}$. in III and ${ }_{\mathrm{a}} \mathrm{V}_{\mathrm{F}}$ in most cases. The maximum value does not exceed $0.034 \mathrm{sec}$. in the groups over 40 year of age in all standard leads.

The mean values do not exceed $0.014 \mathrm{sec}$. in $V_{2}$ through $V_{6}$ leads, and their maximum values are below 0.03 sec. except the 60 year male group. There are no remarkable differences in age or sex.

\section{(3) Duration of Q II (Table VIII)}

The mean valucs of $Q_{\text {II }}$ duration range from $0.027 \mathrm{sec}$. to $0.010 \mathrm{sec}$. in standard leads and unipolar extremity leads except ${ }_{a} V_{R}$ lead. The maximum values are in excess of 0.055 sec. in lead II, III and ${ }_{a} V_{F}$. There are no outstanding differences between sex and ages. 
Vol. 4 NORMAL VALUE OF ELECTROCARDIOGRAM IN THE JAPANESE 145
No. 2 J

Table II. Duration of $\mathbf{P}$ Deflection (in sec.)

\begin{tabular}{|c|c|c|c|c|c|c|c|c|c|}
\hline \multicolumn{10}{|c|}{ Age Group } \\
\hline \multicolumn{2}{|c|}{ Age } & \multicolumn{2}{|c|}{$15 \sim 19$} & \multicolumn{2}{|c|}{$20 \sim 29$} & \multicolumn{2}{|c|}{$30 \sim 39$} & \multicolumn{2}{|c|}{$40 \sim 49$} \\
\hline & & M & $\mathbf{F}$ & M & F & M & F & M & $\mathbf{F}$ \\
\hline Number & f Cases & 61 & 39 & 165 & 169 & 119 & 94 & 153 & 82 \\
\hline Extremity & $M n ., S D$ & $\begin{array}{c}0.098, \\
0.015\end{array}$ & $\begin{array}{r}0.097 \\
0.014\end{array}$ & $\begin{array}{c}0.093 \\
0.013\end{array}$ & $\begin{array}{l}0.089 \\
0.017\end{array}$ & \begin{tabular}{|}
0.097 \\
0.019
\end{tabular} & $\begin{array}{c}0.099 \\
0.017\end{array}$ & $\begin{array}{c}0.095 \\
0.016\end{array}$ & $\begin{array}{l}0.090, \\
0.015\end{array}$ \\
\hline Lead & $\begin{array}{l}\text { Max. } \\
\sim \text { Min. }\end{array}$ & $\begin{array}{l}0.13 \\
\sim 0.06\end{array}$ & $\begin{array}{c}0.13 \\
\sim 0.06\end{array}$ & $\begin{array}{l}0.13 \\
\sim 0.06\end{array}$ & $\begin{array}{l}0.13 \\
\sim 0.04\end{array}$ & $\begin{array}{l}0.17 \\
\sim 0.04\end{array}$ & $\begin{array}{l}0.12 \\
\sim 0.04\end{array}$ & $\begin{array}{l}0.15 \\
\sim 0.04\end{array}$ & $\begin{array}{l}0.15 \\
\sim 0.04\end{array}$ \\
\hline Precordial & Mn., SD & $\begin{array}{c}0.089 \\
0.016\end{array}$ & $\begin{array}{c}0.093 \\
0.016\end{array}$ & $\begin{array}{c}0.091 \\
0.021\end{array}$ & $\begin{array}{r}0.085 \\
0.021\end{array}$ & $\begin{array}{c}0.089 \\
0.022\end{array}$ & $\begin{array}{c}0.088 \\
0.018\end{array}$ & $\begin{array}{c}0.093 \\
0.019\end{array}$ & $\begin{array}{r}0.078, \\
0.019\end{array}$ \\
\hline Lead & $\stackrel{\text { Max. }}{\sim \text { Min. }}$ & $\begin{array}{c}0.13 \\
\sim 0.04\end{array}$ & $\begin{array}{c}0.13 \\
\sim 0.06\end{array}$ & $\begin{array}{r}0.15 \\
\sim 0\end{array}$ & $\begin{array}{l}0.13 \\
\sim 0.02\end{array}$ & $\begin{array}{l}0.13 \\
\sim 0.02\end{array}$ & $\stackrel{0.15}{\sim 0.04} \mid$ & $\begin{array}{l}0.13 \\
\sim 0.02\end{array}$ & $\begin{array}{l}0.13 \\
\sim 0.02\end{array}$ \\
\hline
\end{tabular}

Age Group

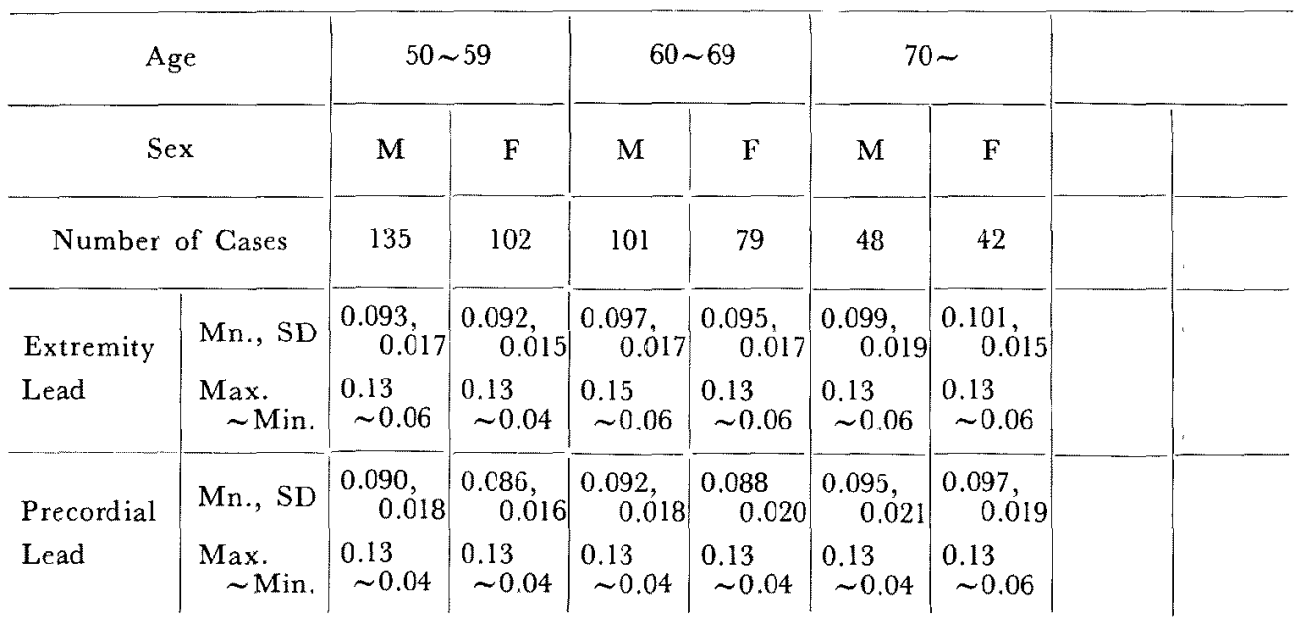

Mn. : Mean value,

Min.: Minimum value,
SD : Standard deviation,

M : Male,
Max: Maximum value,

$\mathrm{F}$ : Female 
Table III. Voltage of

\begin{tabular}{|c|c|c|c|c|c|c|c|}
\hline & & & & & & & Age \\
\hline \multirow{2}{*}{\multicolumn{2}{|c|}{$\begin{array}{l}\text { Age } \\
\text { Sex }\end{array}$}} & \multicolumn{2}{|c|}{$15 \sim 19$} & \multicolumn{2}{|c|}{$20 \sim 29$} & \multicolumn{2}{|c|}{$30 \sim 39$} \\
\hline & & $\mathrm{M}$ & $\mathrm{F}$ & $\mathrm{M}$ & $\mathrm{F}$ & $\mathrm{M}$ & $\mathrm{F}$ \\
\hline \multicolumn{2}{|c|}{ Number of Cases } & 61 & 39 & 165 & 109 & 119 & 94 \\
\hline $\mathrm{I}$ & $\begin{array}{l}\text { Mn., SD } \\
\text { Max. } \sim \text { Min. }\end{array}$ & $\begin{array}{c}0.56,0.33 \\
1.7 \sim 0.6\end{array}$ & $\begin{array}{c}0.51,0.25 \\
1.1 \sim 0.0\end{array}$ & $\begin{array}{c}0.52,0.25 \\
1.1 \sim 0.0\end{array}$ & $\begin{array}{c}0.60,0.31 \\
2.0 \sim 0.0\end{array}$ & $\begin{array}{c}0.48,0.25 \\
1.4 \sim 0.0\end{array}$ & $\begin{array}{c}0.57,0.26 \\
1.4 \sim 0.0\end{array}$ \\
\hline II & $\begin{array}{l}\text { Mn., SD } \\
\text { Max. } \sim \text { Min. }\end{array}$ & $\begin{array}{c}0.92,0.43 \\
2.0 \sim 0.0\end{array}$ & $\begin{array}{c}0.83,0.47 \\
2.0 \sim 0.0\end{array}$ & $\begin{array}{c}1.01,0,47 \\
2.6 \sim 0.0\end{array}$ & $\begin{array}{l}0.80,0.40 \\
2.0 \sim-0.3\end{array}$ & $\begin{array}{l}0.96,0.46 \\
2.0 \sim-0.3\end{array}$ & $\begin{array}{c}0.89,0.35 \\
1.7 \sim 0.0\end{array}$ \\
\hline III & $\begin{array}{l}\text { Mn., SD } \\
\text { Max. } \sim \text { Min. }\end{array}$ & $\begin{array}{l}0.50,0.45 \\
1.7 \sim-1.2\end{array}$ & $\begin{array}{l}0.44,0.46 \\
1.7 \sim-0.3\end{array}$ & $\begin{array}{l}0.65,0.44 \\
2.0 \sim-0.3\end{array}$ & $\begin{array}{l}0.37,0.41 \\
1.4 \sim-0.4\end{array}$ & $\begin{array}{l}0.59,0.47 \\
1.7 \sim-0.9\end{array}$ & $\begin{array}{l}0.39,0.37 \\
1.7 \sim-0.6\end{array}$ \\
\hline$n V_{R}$ & $\begin{array}{l}\text { Mn., SD } \\
\text { Max. } \sim \operatorname{Min}:\end{array}$ & $\left\{\begin{array}{l}-0.62,0.31 \\
-1.2 \sim-0.1\end{array}\right.$ & $\left|\begin{array}{c}-0.62,0.27 \\
0.1 \sim-1.2\end{array}\right|$ & $\begin{array}{l}-0.72,0.34 \\
-0.1 \sim-2.1\end{array}$ & $\begin{array}{l}-0.67,0.30 \\
-0.1 \sim-1.5\end{array}$ & $\begin{array}{c}-0.48,0.49 \\
1.7 \sim-1.5\end{array}$ & $\begin{array}{r}-0.67,0.25 \\
0.1 \sim-1.2\end{array}$ \\
\hline$a V_{L}$ & $\begin{array}{l}\text { Mn., SD } \\
\text { Max. } \sim \text { Min. }\end{array}$ & $\begin{array}{l}0.20,0.36 \\
1.1 \sim-0.9\end{array}$ & $\begin{array}{l}0.15,0.28 \\
1.8 \sim-0.6\end{array}$ & $\begin{array}{l}0.16,0.59 \\
1.1 \sim-1.1\end{array}$ & $\begin{array}{l}0.27,0.37 \\
1.4 \sim-0.1\end{array}$ & $\begin{array}{l}0.12,0.34 \\
1.4 \sim-0.6\end{array}$ & $\begin{array}{l}0.19,0.40 \\
0.8 \sim-2.4\end{array}$ \\
\hline${ }_{a} V_{F}$ & $\begin{array}{l}\text { Mn., SD } \\
\text { Max. Min. }\end{array}$ & $\begin{array}{l}0.71,0.44 \\
2.3 \sim-0.3\end{array}$ & $\begin{array}{c}0.66,0.46 \\
2.0 \sim 0.0\end{array}$ & $\begin{array}{l}0.81,0.52 \\
2.6 \sim-1.4\end{array}$ & $\begin{array}{l}0.54,0.34 \\
1.7 \sim-0.3\end{array}$ & $\begin{array}{l}0.72,0.51 \\
2.0 \sim-1.1\end{array}$ & $\begin{array}{l}0.71,0.35 \\
1.7 \sim-0.3\end{array}$ \\
\hline $\mathrm{V}_{4} \mathrm{R}$ & $\begin{array}{l}\text { Mn., SD } \\
\text { Max. } \sim \text { Min. }\end{array}$ & $\begin{array}{l}0.25,0.31 \\
1.1 \sim-0.6\end{array}$ & $\begin{array}{l}0.26,0.26 \\
1.1 \sim-0.6\end{array}$ & $\begin{array}{l}0.36,0.31 \\
1.7 \sim-0.6\end{array}$ & $\begin{array}{l}0.20,0.25 \\
1.1 \sim-0.6\end{array}$ & $\begin{array}{l}0.28,0.26 \\
0.8 \sim-0.6\end{array}$ & $\begin{array}{l}0.28,0.23 \\
0.8 \sim-0.3\end{array}$ \\
\hline$V_{1}$ & $\begin{array}{l}\text { Mn., SD } \\
\text { Max. Min. }\end{array}$ & $\begin{array}{l}0.59,0.36 \\
1.4 \sim-0.3\end{array}$ & $\begin{array}{c}0.45,0.28 \\
1.1 \sim 0.0\end{array}$ & $\left.\begin{array}{l}0.54,0.37 \\
2.0 \sim-0.9\end{array}\right)$ & $\begin{array}{r}0.4,0.30 \\
1.1 \sim-0.6\end{array}$ & $\begin{array}{l}0.40,0.30 \\
1.1 \sim-0.9\end{array}$ & $\begin{array}{l}0.41,0.31 \\
1.1 \sim-0.6\end{array}$ \\
\hline$V_{s}$ & $\begin{array}{l}\text { Mn., SD } \\
\text { Max. } \sim \text { Min. }\end{array}$ & $\begin{array}{c}0.68,0.42 \\
2.0 \sim 0.0\end{array}$ & $\begin{array}{l}0.50,0.2 \\
1.4 \sim 0.0\end{array}$ & $\begin{array}{c}0.60,0.34 \\
1.7 \sim-0.3\end{array}$ & $\begin{array}{l}0.49,0.32 \\
1.4 \sim-0.6\end{array}$ & $\begin{array}{l}0.44,0.31 \\
1.4 \sim-0.6\end{array}$ & $\begin{array}{l}0.50,0.32 \\
1.7 \sim-0.3\end{array}$ \\
\hline$V_{3}$ & $\begin{array}{l}\text { Mn., SD } \\
\text { Max. Min. }\end{array}$ & $\begin{array}{c}0.63,0.36 \\
1.7 \sim 0.0\end{array}$ & $\begin{array}{c}0.51,0.28 \\
1.1 \sim 0.0\end{array}$ & $\begin{array}{c}0.61,0.35 \\
1.7 \sim 0.0\end{array}$ & $\begin{array}{l}0.50,0.28 \\
1.1 \sim-0.6\end{array}$ & $\begin{array}{c}0.44,0.25 \\
1.4 \sim 0.0\end{array}$ & $\begin{array}{c}0.50,0.28 \\
\text { I. } 7 \sim 0.0\end{array}$ \\
\hline $\mathrm{V}_{4}$ & $\begin{array}{l}\text { Mn., } \mathrm{SD} \\
\text { Max. } \sim \text { Min. }\end{array}$ & $\begin{array}{c}0.60,0.34 \\
1.7 \sim 0.0\end{array}$ & $\begin{array}{c}0.43,0.24 \\
1.1 \sim 0.0\end{array}$ & $\begin{array}{c}0.59,0.33 \\
1.7 \sim 0.0\end{array}$ & $\begin{array}{c}0.42,0.24 \\
1.1 \sim 0.6\end{array}$ & $\begin{array}{c}0.45,0.26 \\
1.4 \sim 0.0\end{array}$ & $\begin{array}{c}0.49,0.30 \\
1.7 \sim 0.0\end{array}$ \\
\hline$V_{3}$ & $\begin{array}{l}\text { Mn., SD } \\
\text { Max. } \sim \text { Min. }\end{array}$ & $\begin{array}{c}0.50,0.37 \\
1.4 \sim 0.0\end{array}$ & $\begin{array}{c}0.40,0.26 \\
1.1 \sim 0.0\end{array}$ & $\begin{array}{c}0.56,0.33 \\
2.0 \sim 0.0\end{array}$ & $\begin{array}{l}0.39,0.25 \\
1.1 \sim-0.3\end{array}$ & $\begin{array}{c}0.42,0.24 \\
1.4 \sim 0.0\end{array}$ & $\begin{array}{c}0.74,0.40 \\
1.7 \sim 0.0\end{array}$ \\
\hline$V_{B}$ & $\begin{array}{l}\text { Mn., SD } \\
\text { Max. } \sim \text { Min. }\end{array}$ & $\begin{array}{c}0.47,0.34 \\
1.7 \sim 0.0\end{array}$ & $\begin{array}{c}0.29,0.24 \\
1.1 \sim 0.0\end{array}$ & $\begin{array}{c}0.50,0.33 \\
1.7 \sim 0.0\end{array}$ & $\begin{array}{l}0.36,0.25 \\
1.4 \sim-0.3\end{array}$ & $\begin{array}{c}0.35,0.21 \\
1.1 \sim 0.0\end{array}$ & $\begin{array}{c}0.38,0.26 \\
1.7 \sim 0.0\end{array}$ \\
\hline
\end{tabular}

Mn. : Mean value,

SD: Standard deviation,

Max.: Maximum value,

Min.: Minimum value, $M$ : Male, F: Female, 
$\mathrm{P}$ Deflection (in $\mathrm{mm}$.)

Group (yr.)

\begin{tabular}{|c|c|c|c|c|c|c|c|}
\hline \multicolumn{2}{|c|}{$40 \sim 49$} & \multicolumn{2}{|c|}{$50 \sim 59$} & \multicolumn{2}{|c|}{$60 \sim 69$} & \multicolumn{2}{|c|}{$70 \sim$} \\
\hline M & $\mathrm{F}$ & M & $\mathrm{F}$ & M & $\mathrm{F}$ & $\mathrm{M}$ & $\mathrm{F}$ \\
\hline 153 & 82 & 135 & 102 & $(0)$ & 79 & 48 & 42 \\
\hline $0.54,0.28$ & $0.52,0.24$ & $0.48,0.28$ & $0.60,0.25$ & $0.43,0.27$ & $0.59,0.31$ & $0.43,0.23$ & $0.62,0.33$ \\
\hline $2.2 \sim 0.0$ & $1.1 \sim 0.0$ & $1.4 \sim 0.0$ & $1.4 \sim 0.0$ & $1.1 \sim 0.0$ & $1.7 \sim 0.0$ & $1.1 \sim 0.0$ & $1.4 \sim 0.0$ \\
\hline $1.00,0.24$ & $0.83,0.37$ & $1.00,0.41$ & $0.86,0.42$ & $1.10,0.43$ & $0.94,0.48$ & $0.97,0.48$ & $0.89,0.39$ \\
\hline $2.3 \sim 0.0$ & $1.7 \sim 0.0$ & $2.0 \sim 0.0$ & $2.3 \sim 0.0$ & $2.3 \sim 0.3$ & $2.3 \sim 0.0$ & $2.0 \sim 0.0$ & $2.0 \sim 0.0$ \\
\hline $0.65,0.44$ & $0.45,0.38$ & $0.65,0.52$ & $0.46,0.42$ & $0.69,0.45$ & $0.42,0.55$ & $0.65,0.44$ & $0.40,0.65$ \\
\hline $2.3 \sim-0.8$ & $1.7 \sim-0.6$ & $4.1 \sim-0.6$ & $1.4 \sim-0.9$ & $2.3 \sim-0.6$ & $2.0 \sim-1.5$ & $1.7 \sim-0.3$ & $1.7 \sim-2.1$ \\
\hline $0.51,0.46$ & $-0.65,0.25$ & $-0.70,0.60$ & $-0.68,0.31$ & $0.67,0.44$ & $-0.68,0.42$ & $-0.64,0.41$ & $-0.50,0.61$ \\
\hline $1.2 \sim 0.0$ & $0.1 \sim-1.2$ & $0.8 \sim-1.6$ & $0.5 \sim-1.5$ & $1.1 \sim-1.8$ & $0.8 \sim-2.1$ & $0.5 \sim-1.5$ & $1.1 \sim-1.5$ \\
\hline $0.09,0.28$ & $0.18,0.34$ & $0.06,0.39$ & $0.25,0.33$ & $-0.05,0.40$ & $0.28,0.43$ & $0.10,0.35$ & $0.25,0.43$ \\
\hline $1.1 \sim-1.4$ & $1.1 \sim-0.6$ & $1.1 \sim-0.9$ & $1.1 \sim-0.6$ & $1.1 \sim-1.2$ & $1.7 \sim-1.2$ & $1.1 \sim-0.6$ & $1.1 \sim-0.6$ \\
\hline $0.81,0.19$ & $0.61,0.35$ & $0.79,0.42$ & $0.59,0.39$ & $0.83,0.43$ & $0.62,0.46$ & $0.76,0.44$ & $0.59,0.46$ \\
\hline $2.3 \sim-0.6$ & $1.7 \sim-0.3$ & $1.7 \sim-0.3$ & $2.0 \sim 0.0$ & $2.6 \sim 0.2$ & $2.3 \sim-0.6$ & $2.0 \sim-0.3$ & $1.7 \sim-1.2$ \\
\hline $0.34,0.31$ & $0.30,0.26$ & $0.30,0.29$ & $0.34,0.34$ & $0.24,0.37$ & $0.13,0.04$ & $0.27,0.40$ & $0.29,0.31$ \\
\hline $1.4 \sim-0.3$ & $1.1 \sim-0.6$ & $1.4 \sim-0.6$ & $1.1 \sim-0.9$ & $1.1 \sim-0.9$ & $1.4 \sim-1.5$ & $1.1 \sim-0.6$ & $1.1 \sim-0.3$ \\
\hline $0.28,0.12$ & $0.39,0.28$ & $0.29,0.40$ & $0.43,0.31$ & $0.32,0.41$ & $0.25,0.49$ & $0.42,0.41$ & $0.20,0.46$ \\
\hline $1.1 \sim-1.5$ & $1.3 \sim-0.9$ & $1.4 \sim-1.2$ & $1.1 \sim-0.6$ & $1.4 \sim-1.4$ & $1.7 \sim-1.4$ & $1.7 \sim-0.6$ & $1.1 \sim-1.2$ \\
\hline $0.42,0.34$ & $0.47,0.30$ & $0.14,0.27$ & $0.46,0.26$ & $0.44,0.33$ & $0.41,0.30$ & $0.56,0.36$ & $0.36,0.37$ \\
\hline $1.4 \sim-1.4$ & $1.1 \sim 0.6$ & $1.4 \sim-0.3$ & $1.1 \sim-0.3$ & $1.7 \sim-0.9$ & $1.4 \sim-0.3$ & $1.4 \sim 0.0$ & $1.1 \sim-0.9$ \\
\hline $0.52,0.28$ & $0.51,0.28$ & $0.43,0.24$ & $0.50,0.25$ & $0.49,0.36$ & $0.36,0.30$ & $0.52,0.30$ & $0.51,0.30$ \\
\hline $1.7 \sim 0.0$ & $2.0 \sim 0.0$ & $1.1 \sim 0.0$ & $1.1 \sim 0.0$ & $2.6 \sim-0.3$ & $1.7 \sim 0$ & $1.7 \sim 0.0$ & $1.4 \sim 0.0$ \\
\hline $0.54,0.29$ & $0.46,1.22$ & $0.46,0.23$ & $0.51,0.21$ & $0.51,0.27$ & $0.51,0.31$ & $0.53,0.27$ & $0.49,0.3$ \\
\hline $1.7 \sim 0.0$ & $1.1 \sim 0.0$ & $1.1 \sim 0.0$ & $1.1 \sim 0.0$ & $1.4 \sim 0.0$ & $1.4 \sim 0.0$ & $1.1 \sim 0.0$ & $1.4 \sim 0.0$ \\
\hline $0.49,0.27$ & $0.42,0.17$ & $0.44,0.24$ & $0.44,0.23$ & $0.44,0.24$ & $0.45,0.23$ & $0.43,0.26$ & $0.48,0.25$ \\
\hline $1.4 \sim 0.0$ & $0.8 \sim 0.0$ & $1.4 \sim 0.0$ & $1.1 \sim 0.0$ & $1.1 \sim 0.0$ & $1.1 \sim 0.0$ & $1.1 \sim 0.0$ & $1.1 \sim 0.0$ \\
\hline $0.47,0.29$ & $0.36,0.17$ & $0.42,0.24$ & $0.41,0.21$ & $0.42,0.25$ & $0.42,0.32$ & $0.38,0.25$ & $0.46,0.28$ \\
\hline $1.7 \sim 0.0$ & $1.1 \sim 0.0$ & $1.1 \sim 0.0$ & $1.1 \sim 0.0$ & $1.1 \sim 0.0$ & $0.6 \sim 0.0$ & $1.7 \sim 0.0$ & $1.4 \sim 0.0$ \\
\hline
\end{tabular}


Table IV.

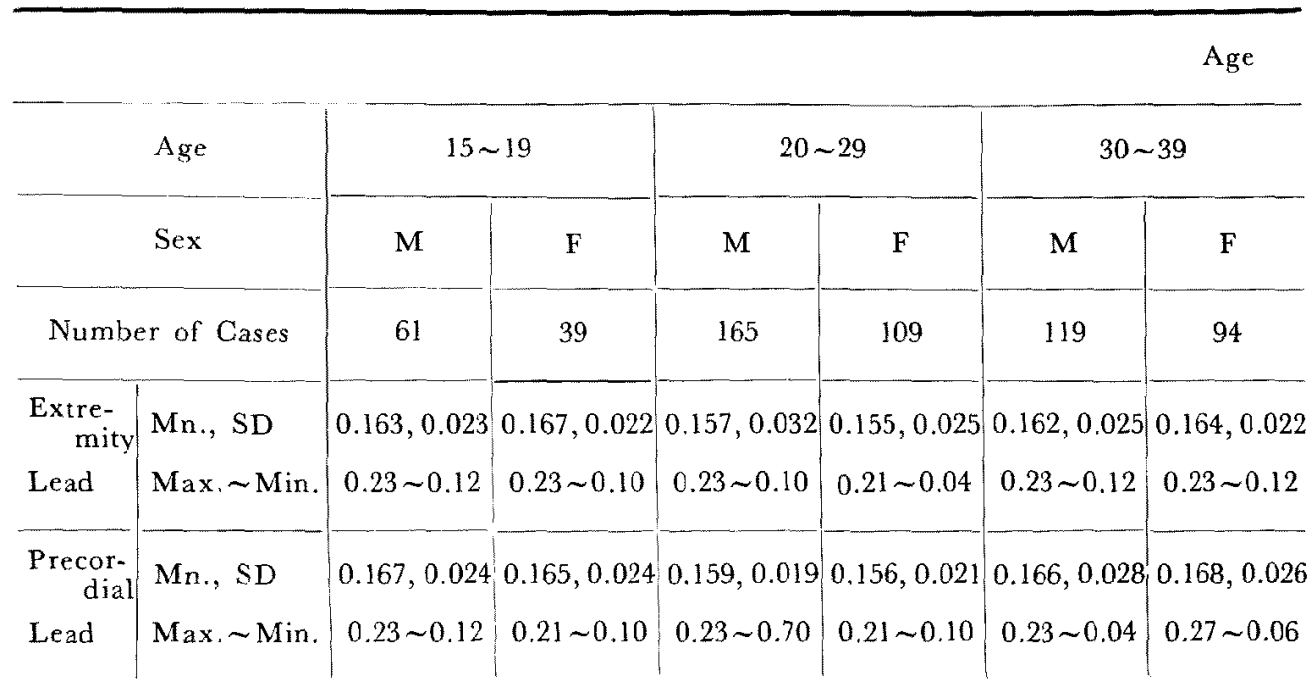

Table V.

\begin{tabular}{|c|c|c|c|c|c|c|c|}
\hline & & & & & & & Age \\
\hline \multirow{2}{*}{\multicolumn{2}{|c|}{$\underbrace{\text { Age }}_{\text {Sex }}$}} & \multicolumn{2}{|c|}{$15 \sim 19$} & \multicolumn{2}{|c|}{$20 \sim 29$} & \multicolumn{2}{|c|}{$30 \sim 39$} \\
\hline & & $\mathbf{M}$ & $\mathrm{F}$ & M & $F$ & M & $\mathrm{F}$ \\
\hline \multicolumn{2}{|c|}{ Number of Cases } & 61 & 39 & 165 & 109 & 119 & 94 \\
\hline $\begin{array}{c}\text { Extre- } \\
\text { mity }\end{array}$ & $\mathrm{Mn}, \mathrm{SD}$ & $0.090,0.013$ & $0.067,0.010$ & $0.080,0.015$ & $0.074,0.015$ & $0.082,0.015$ & $0.069,0.015$ \\
\hline Lead & Max, $\sim \operatorname{Min}$. & $0.150 \sim 0.06$ & $0.09 \sim 0.05$ & $0.13 \sim 0.04$ & $0.14 \sim 0.04$ & $0.11 \sim 0.04$ & $0.12 \sim 0.04$ \\
\hline $\begin{array}{r}\text { Precor } \\
\text { dial }\end{array}$ & $\mathrm{Mn} ., \mathrm{SD}$ & $0.096,0.015$ & $0.075,0.010$ & $0.087,0.014$ & $0.084,0.014$ & $0.085,0.012$ & $0.080,0.013$ \\
\hline Lead & Max. $\sim \operatorname{Min}$ & $0.19 \sim 0.06$ & $0.09 \sim 0.06$ & $0.16 \sim 0.03$ & $0.14 \sim 0.04$ & $0.11 \sim 0.05$ & $0.12 \sim 0.05$ \\
\hline
\end{tabular}

The mean values of $Q_{11}$ duration in lead $V_{4}, V_{5}$ and $V_{6}$ do not exceed $0.03 \mathrm{sec}$. Most maximum values are within $0.044 \mathrm{sec}$. There is no remarkable difference due to age and sex. 


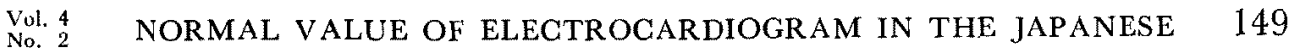
$\mathrm{P}-\mathrm{Q}$ Interval (in sec.)

Group (yr.)

\begin{tabular}{|c|c|c|c|c|c|c|c|}
\hline \multicolumn{2}{|c|}{$40 \sim 49$} & \multicolumn{2}{|c|}{$50 \sim 59$} & \multicolumn{2}{|c|}{$60 \sim 69$} & \multicolumn{2}{|c|}{$70 \sim$} \\
\hline $\mathbf{M}$ & $\mathbf{F}$ & M & $\mathrm{F}$ & $\mathbf{M}$ & F & $\mathbf{M}$ & $\mathrm{F}$ \\
\hline 153 & 82 & 135 & 102 & 101 & 79 & 48 & 42 \\
\hline $0.167,0.026$ & $0.159,0.023$ & $0.164,0.022$ & $0.164,0.025$ & $0.171,0.031$ & $0.165,0.024$ & $0.170,0.032$ & $0.162,0.020$ \\
\hline $0.25 \sim 0.06$ & $0.23 \sim 0.12$ & $0.23 \sim 0.10$ & $0.23 \sim 0.10$ & $0.39 \sim 0.10$ & $0.25 \sim 0.12$ & $0.23 \sim 0.10$ & $0.21 \sim 0.10$ \\
\hline $0.164,0.024$ & $0.156,0.022$ & $0.165,0.022$ & $0.161,0.024$ & $0.168,0.031$ & $0.162,0.024$ & $0.170,0.032$ & $0.168,0.021$ \\
\hline $0.23 \sim 0.08$ & $0.21 \sim 0.10$ & $0.23 \sim 0.10$ & $0.23 \sim 0.10$ & $0.37 \sim 0.10$ & $0.27 \sim 0.12$ & $0.27 \sim 0.10$ & $0.21 \sim 0.12$ \\
\hline
\end{tabular}

QRS Duration (in sec.)

Group (yr.)

\begin{tabular}{c|c|c|c|c|c|c|c}
\hline \multicolumn{2}{c|}{$40 \sim 49$} & \multicolumn{2}{|c|}{$50 \sim 59$} & \multicolumn{2}{|c|}{$60 \sim 69$} & \multicolumn{2}{|c}{$70 \sim$} \\
\hline$M$ & $\mathrm{~F}$ & $\mathrm{M}$ & $\mathrm{F}$ & $\mathrm{M}$ & $\mathrm{F}$ & $\mathrm{M}$ & $\mathrm{F}$ \\
\hline 153 & 82 & 135 & 102 & 101 & 79 & 48 & 42 \\
\hline $0.081,0.016$ & $0.074,0.028$ & $0.076,0.012$ & $0.075,0.012$ & $0.080,0.013$ & $0.077,0.013$ & $0.085,0.019$ & $0.079,0.013$ \\
$0.18 \sim 0.04$ & $0.12 \sim 0.05$ & $0.12 \sim 0.04$ & $0.12 \sim 0.04$ & $0.12 \sim 0.05$ & $0.11 \sim 0.05$ & $0.18 \sim 0.05$ & $0.11 \sim 0.05$ \\
\hline $0.087,0.015$ & $0.081,0.024$ & $0.086,0.012$ & $0.081,0.010$ & $0.083,0.013$ & $0.084,0.014$ & $0.089,0.017$ & $0.082,0.009$ \\
$0.17 \sim 0.05$ & $0.11 \sim 0.05$ & $0.12 \sim 0.05$ & $0.11, \sim 0.06$ & $0.12 \sim 0.06$ & $0.12 \sim 0.06$ & $0.18 \sim 0.07$ & $0.10 \sim 0.07$
\end{tabular}

\section{$R$ Deflection}

Voltage of $R$ Deflection (Table IX)

The mean values of $R$ deflections of lead $I$ are in the narrow range indicating from $5.30 \mathrm{~mm}$. to $6.55 \mathrm{~mm}$. but their values in lead II, III and ${ }_{\mathrm{a}} \mathrm{V}_{\mathrm{F}}$ have a tendency to decrease with ages. The mean values in lead II 
are the largest among the standard leads, and range from $7.78 \mathrm{~mm}$. to $15.35 \mathrm{~mm}$.

The mean values of $\mathrm{R}$ deflection are largest in the males of 10 and 20 year age groups in all precordial leads. The voltages are mostly larger in the male group than in the female of each age group. The maximum value of normal range in $\mathrm{V}_{1}$ is from $11.46 \mathrm{~mm}$. to $4.71 \mathrm{~mm}$. in the male group, and is from $8.85 \mathrm{~mm}$. to $4.26 \mathrm{~mm}$. in the female group. In lead $\mathrm{V}_{5}$, it ranges from $33.33 \mathrm{~mm}$. to $27.72 \mathrm{~mm}$. in the male group, and $29.09 \mathrm{~mm}$. to $22.33 \mathrm{~mm}$. in the female group.

\section{$S$ Deflection (Table X)}

The mean values of $\mathrm{S}$ deflections do not exceed $3 \mathrm{~mm}$. in all standard leads. The mean and maximum values increase a little with age in lead III and ${ }_{a} V_{F}$.

The value of $\mathrm{S}$ is largest in $\mathrm{V}_{2}$ among the precordial leads. In lead $V_{2}$ and $V_{3}$, the mean values are larger in male groups. There is no remarkable differences with age and sex in other leads.

\section{$S-T$ Segment}

Because of gradual sloping of the curve from QRS to $\mathrm{S}-\mathrm{T}$ segment, sometimes there are some difficulties in deciding the " $J$ " point. The displacement of S-T segment is measured at the " $\mathrm{J}$ " point and at the point $0.04 \mathrm{sec}$. apart from the " $\mathrm{J}$ " point which is symbolized as ST-0.04 segment.

\section{(1) " $J$ " point (Table XI)}

In lead $I$, mean values are almost near the zero line in all age groups and their SD is below $0.25 \mathrm{~mm}$. Calculated normal limit ranges from 0.50 to $-0.50 \mathrm{~mm}$. These values increase in lead II, III and ${ }_{\mathrm{a}} \mathrm{V}_{\mathrm{F}}$, in which maximum depression is about $0.8 \mathrm{~mm}$. and not more than $1 \mathrm{~mm}$. There are no remarkable differences with age and sex in them.

In most cases, the mean values are positive in the precordial leads. Male groups usually have more positive value than female groups, through $V_{1}$ to $V_{4}$ lead. The maximum elevation of " $\mathrm{J}$ " point is obtained from $\mathrm{V}_{2}$ in most age groups and reaches to $2.9 \mathrm{~mm}$. in the 20 year male group.

Maximum depression of normal limit in each precordial lead regardless of age is as follows; $V_{1},-0.85 \mathrm{~mm} ., V_{3},-2.14 \mathrm{~mm} ., \mathrm{V}_{4},-1.23 \mathrm{~mm} ., \mathrm{V}_{5}$, $-0.99 \mathrm{~mm}$., $\mathrm{V}_{6},-0.84 \mathrm{~mm}$. 


\section{(2) S-T 0.04 Segment (Table XII)}

S-T 0.04 segment shows almost the same tendency as described in " $J$ " point except its values, which incline to take more positive value than "J" point.

\section{$T$ Defletion (Table XIII)}

The mean values of lead I range from $3.2 \mathrm{~mm}$. to $1.5 \mathrm{~mm}$. according to ages. In almost all cases of each decade except the 70 year male group, positive $\mathrm{T}$ deflection is found in lead $\mathrm{I}$. The lower limit of normal value ranges from $1.0 \mathrm{~mm}$. to $0.0 \mathrm{~mm}$. in male group, and $0.7 \mathrm{~mm}$. to $0.0 \mathrm{~mm}$. in female group.

In lead II, mean values range from $4.7 \mathrm{~mm}$. to $2.1 \mathrm{~mm}$. and the values in lead III and ${ }_{a} V_{F}$ are smaller than in lead II. In the large number of cases, male groups show larger values than female groups, and the values decrease with age.

Voltages in precordial leads are as follows.

$\mathrm{V}_{1}$ : The mean values of male groups are all positive. In female of $10,20,30$, and 50 year groups, the mean value shows negative $T$ wave. The lower limit of normal ranges of $\mathrm{T}$ wave is usually negative in all ages and in both sexes.

$\mathrm{V}_{2}$ : The mean values are positive in all age groups regardless of sex. Young male groups have larger mean value. As for the lower limit of the normal range, the fcmale groups indicate the negative value regardless of age, but in the male group of 10,20,40,50 and 70 year, they have positive values.

$\mathrm{V}_{3}$ : Mean value in $\mathrm{V}_{3}$ is slightly larger than that in $\mathrm{V}_{2}$. Male groups have positive values of $T$ deflections in both mean value and lower limit. In the female groups, mean values are all positive, but lower limit is slightly negative in almost all cases except 10 year group.

$\mathrm{V}_{4}$ : The feature in $\mathrm{V}_{4}$ is almost the same as that in $V_{3}$. Even in $\mathrm{V}_{4}$ lead, males of 30 year group and females of 30 and 50 year groups show the negative value of lower limit.

$\mathrm{V}_{5}$ : The mean values are mostly positive in each decade, and lower limits range from $1.12 \mathrm{~mm}$. to $-0.34 \mathrm{~mm}$., and are isoelectric in most cases.

$V_{6}$ : In $V_{6}$ the mean values of $T$ deflections show no remarkable differences with sex, but a tendency to decrease with age. All the mean values are positive. The lower limits of normal values are all positive or isoelectric, ranging from $1.0 \mathrm{~mm}$. to $0 \mathrm{~mm}$. 
Table VI. Voltage of

\begin{tabular}{|c|c|c|c|c|c|c|c|}
\hline & & & & & & & Age \\
\hline \multirow{2}{*}{\multicolumn{2}{|c|}{$\begin{array}{l}\text { Age } \\
\text { Sex }\end{array}$}} & \multicolumn{2}{|c|}{$15 \sim 19$} & \multicolumn{2}{|c|}{$20 \sim 29$} & \multicolumn{2}{|c|}{$30 \sim 39$} \\
\hline & & $\mathbf{M}$ & $\mathbf{F}$ & $\mathbf{M}$ & $\mathbf{F}$ & $\mathbf{M}$ & $\mathrm{F}$ \\
\hline \multicolumn{2}{|c|}{ Number of Cases } & 61 & 39 & 165 & 109 & 119 & 94 \\
\hline I & $\begin{array}{l}\text { Mn. } \\
\text { Max. Min. }\end{array}$ & $\begin{array}{l}0.3 \\
1.9 \sim 0\end{array}$ & $\begin{array}{l}0.28 \\
1.5 \sim 0\end{array}$ & $\begin{array}{l}0.32 \\
4.1 \sim 0\end{array}$ & $\begin{array}{l}0.22 \\
1.5 \sim 0\end{array}$ & $\begin{array}{l}0.37 \\
2.47 \sim 0\end{array}$ & $\begin{array}{l}0.18 \\
1.8 \sim 0\end{array}$ \\
\hline II & $\begin{array}{l}\text { Mn. } \\
\text { Max. Min. }\end{array}$ & $\begin{array}{l}0.66 \\
3.2 \sim 0\end{array}$ & $\begin{array}{l}0.44 \\
2.5 \sim 0\end{array}$ & $\begin{array}{l}0.73 \\
9.2 \sim 0\end{array}$ & $\begin{array}{l}0.46 \\
2.7 \sim 0\end{array}$ & $\begin{array}{l}0.52 \\
3.2 \sim 0\end{array}$ & $\begin{array}{l}0.26 \\
1.9 \sim 0\end{array}$ \\
\hline III & $\begin{array}{l}\text { Mn. } \\
\text { Max. Min. }\end{array}$ & $\begin{array}{l}0.69 \\
3.0 \sim 0\end{array}$ & $\begin{array}{l}0.51 \\
3.2 \sim 0\end{array}$ & $\begin{array}{l}0.72 \\
8.8 \sim 0\end{array}$ & $\begin{array}{l}0.66 \\
3.2 \sim 0\end{array}$ & $\begin{array}{l}0.56 \\
2.9 \sim 0\end{array}$ & $\begin{array}{l}0.48 \\
2.11 \sim 0\end{array}$ \\
\hline${ }_{a} V_{R}$ & $\begin{array}{l}\text { Mn. } \\
\text { Max. Min. }\end{array}$ & $\begin{array}{l}9.27 \\
2.0 \sim 0 \\
\quad(30) \\
\end{array}$ & $\begin{array}{l}7.31 \\
10.1 \sim 5.5\end{array}$ & $\begin{array}{l}7.38 \\
13.5 \sim 0\end{array}$ & $\begin{array}{c}8.45 \\
14.3 \sim 2.8 \\
-(47) \\
\end{array}$ & $\begin{array}{l}3.35 \\
12.9 \sim 0 \\
\end{array}$ & $\begin{array}{l}6.74 \\
11.5 \sim 0\end{array}$ \\
\hline${ }_{\mathrm{a}} V_{\mathrm{L}}$ & $\begin{array}{l}\text { Mn. } \\
\text { Max. Min. }\end{array}$ & $\begin{array}{l}0.28 \\
2.0 \sim 0\end{array}$ & $\begin{array}{l}0.26 \\
2.1 \sim 0\end{array}$ & $\begin{array}{l}0.33 \\
4.0 \sim 0\end{array}$ & $\begin{array}{l}0.20 \\
3.0 \sim 0\end{array}$ & $\begin{array}{l}0.15 \\
3.2 \sim 0\end{array}$ & $\begin{array}{l}0.29 \\
4.4 \sim 0\end{array}$ \\
\hline${ }_{\mathrm{a}} V_{\mathrm{F}}$ & $\begin{array}{l}\text { Mn. } \\
\text { Max. Min. }\end{array}$ & $\begin{array}{l}0.59 \\
3.8 \sim 0\end{array}$ & $\begin{array}{l}0.47 \\
2.4 \sim 0\end{array}$ & $\begin{array}{l}0.60 \\
5.8 \sim 0\end{array}$ & $\begin{array}{l}0.40 \\
2.5 \sim 0\end{array}$ & $\begin{array}{l}0.50 \\
3.0 \sim 0\end{array}$ & $\begin{array}{l}0.35 \\
3.4 \sim 0\end{array}$ \\
\hline $\mathrm{V}_{4} \mathrm{R}$ & $\begin{array}{l}\text { Mn. } \\
\text { Max. } \sim \text { Min. }\end{array}$ & $\begin{array}{l}0.03 \\
1.5 \sim 0 \\
\quad(31) \\
\end{array}$ & $\begin{array}{l}0 \\
0\end{array}$ & $\begin{array}{l}0.15 \\
1.4 \sim 0 \\
\quad(117)\end{array}$ & $\begin{array}{l}0.03 \\
1.2 \sim 0 \\
(95) \\
\end{array}$ & $\begin{array}{l}0.11 \\
4.6 \sim 0\end{array}$ & $\begin{array}{l}0.01 \\
0.2 \sim 0 \\
\quad(75) \\
\end{array}$ \\
\hline$V_{1}$ & $\begin{array}{l}\text { Mn. } \\
\text { Max. Min. }\end{array}$ & $\begin{array}{l}0.03 \\
1.2 \sim 0\end{array}$ & $\begin{array}{l}0 \\
0\end{array}$ & $\begin{array}{l}0.01 \\
1.1 \sim 0\end{array}$ & $\begin{array}{l}0 \\
0\end{array}$ & $\begin{array}{l}0.01 \\
1.6 \sim 0\end{array}$ & $\begin{array}{l}0 \\
0\end{array}$ \\
\hline$V_{2}$ & $\begin{array}{l}\mathrm{Mn} . \\
\mathrm{Max} . \sim \operatorname{Min} .\end{array}$ & $\begin{array}{l}0.04 \\
1.8 \sim 0\end{array}$ & $\begin{array}{l}0 \\
0\end{array}$ & $\begin{array}{l}0.01 \\
0.1 \sim 0\end{array}$ & $\begin{array}{l}0.01 \\
0.3 \sim 0\end{array}$ & $\begin{array}{l}0.01 \\
1.1 \sim 0\end{array}$ & 0 \\
\hline $\mathrm{V}_{3}$ & $\begin{array}{l}\mathrm{Mn} . \\
\mathrm{Max} . \sim \mathrm{Min} .\end{array}$ & $\begin{array}{l}0.03 \\
1.5 \sim 0\end{array}$ & $\begin{array}{l}0 \\
0\end{array}$ & $\begin{array}{l}0.01 \\
1.1 \sim 0\end{array}$ & $\begin{array}{l}0 \\
0\end{array}$ & $\begin{array}{l}0.01 \\
0.5 \sim 0\end{array}$ & $\begin{array}{l}0.01 \\
0.1 \sim 0\end{array}$ \\
\hline $\mathrm{V}_{4}$ & $\begin{array}{l}\operatorname{Mn} . \\
\operatorname{Max} . \sim \operatorname{Min} .\end{array}$ & $\begin{array}{l}0.35 \\
3.5 \sim 0\end{array}$ & $\begin{array}{l}0.16 \\
3.0 \sim 0\end{array}$ & $\begin{array}{l}0.31 \\
5.8 \sim 0\end{array}$ & $\begin{array}{l}0.13 \\
1.1 \sim 0\end{array}$ & $\begin{array}{l}0.4 \\
3.1 \sim 0\end{array}$ & $\begin{array}{l}0.01 \\
1.0 \sim 0\end{array}$ \\
\hline$V_{5}$ & $\begin{array}{l}\text { Mn. } \\
\text { Max. } \sim \operatorname{Min} .\end{array}$ & $\begin{array}{l}1.01 \\
5.0 \sim 0\end{array}$ & $\begin{array}{l}0.39 \\
4.1 \sim 0\end{array}$ & $\begin{array}{l}0.87 \\
5.2 \sim 0\end{array}$ & $\begin{array}{l}0.33 \\
2.0 \sim 0\end{array}$ & $\begin{array}{l}0.70 \\
3.4 \sim 0\end{array}$ & $\begin{array}{l}0.04 \\
1.3 \sim 0\end{array}$ \\
\hline $\mathrm{V}_{6}$ & $\begin{array}{l}\text { Mn. } \\
\text { Max. } \sim \operatorname{Min} .\end{array}$ & $\begin{array}{l}0.95 \\
4.0 \sim 0\end{array}$ & $\begin{array}{l}0.56 \\
3.9 \sim 0\end{array}$ & $\begin{array}{l}0.92 \\
4.8 \sim 0\end{array}$ & $\begin{array}{l}0.45 \\
2.0 \sim 0\end{array}$ & $\begin{array}{l}0.58 \\
3.1-0\end{array}$ & $\begin{array}{l}0.39 \\
1.8 \sim 0\end{array}$ \\
\hline
\end{tabular}

( ): Number of cases in which measurement was made. 
Vol. 4 NORMAL VALUE OF ELEGTROCARDIOGRAM IN THE JAPANESE 153
No. 2 S Q Deflection (in $\mathrm{mm}$.)

Group (yr.)

\begin{tabular}{|c|c|c|c|c|c|c|c|}
\hline \multicolumn{2}{|c|}{$40 \sim 49$} & \multicolumn{2}{|c|}{$50 \sim 59$} & \multicolumn{2}{|c|}{$60 \sim 69$} & \multicolumn{2}{|c|}{$70 \sim$} \\
\hline $\mathbf{M}$ & $\mathrm{F}$ & $\mathbf{M}$ & $\mathrm{F}$ & $\mathbf{M}$ & $\mathbf{F}$ & $\mathbf{M}$ & $\mathbf{F}$ \\
\hline 153 & 82 & 135 & 102 & 101 & 79 & 48 & 42 \\
\hline 0.27 & 0.24 & 0.25 & 0.33 & 0.27 & 0.30 & 0.26 & 0.39 \\
\hline $2.1 \sim 0$ & $2.0 \sim 0$ & $1.7 \sim 0$ & $2.0 \sim 0$ & $2.3 \sim 0$ & $2.3 \sim 0$ & $2.0 \sim 0$ & $1.7 \sim 0$ \\
\hline 0.34 & 0.30 & 0.44 & 0.29 & 0.35 & 0.21 & 0.28 & 0.30 \\
\hline $2.4 \sim 0$ & $2.1 \sim 0$ & $5.6 \sim 0$ & $1.1 \sim 0$ & $2.0 \sim 0$ & $1.7 \sim 0$ & $1.1 \sim 0$ & $1.7 \sim 0$ \\
\hline 0.40 & 0.42 & 0.54 & 0.39 & 0.39 & 0.40 & 0.43 & 0.23 \\
\hline $2.9 \sim 0$ & $5.6 \sim 0$ & $11.0 \sim 0$ & $4.4 \sim 0$ & $2.0 \sim 0$ & $2.3 \sim 0$ & $2.6 \sim 0$ & $2.9 \sim 0$ \\
\hline 6.46 & 6.57 & 3.68 & 3.79 & 3.89 & 4.12 & 3.56 & 2.57 \\
\hline $18.2 \sim 0$ & $14.5 \sim 0.8$ & $14.0 \sim 0$ & $10.9 \sim 0$ & $12.9 \sim 0$ & $9.9 \sim 0$ & $16.9 \sim 0$ & $1.39 \sim 0$ \\
\hline 0.47 & 0.30 & 0.45 & 0.40 & 0.50 & 0.46 & 0.74 & 0.59 \\
\hline $5.2 \sim 0$ & $1.8 \sim 0$ & $3.8 \sim 0$ & $2.6 \sim 0$ & $4.1 \sim 0$ & $4.1 \sim 0$ & $5.3 \sim 0$ & $3.2 \sim 0$ \\
\hline 0.33 & 0.23 & 0.43 & 0.30 & 0.30 & 0.24 & 0.32 & 0.21 \\
\hline $3.1 \sim 0$ & $2.2 \sim 0$ & $5.3 \sim 0$ & $2.6 \sim 0$ & $2.0 \sim 0$ & $1.7 \sim 0$ & $1.1 \sim 0$ & $1.4 \sim 0$ \\
\hline $\begin{array}{l}0.15 \\
6.8 \sim 0\end{array}$ & $\begin{array}{l}0.18 \\
4.3 \sim 0 \\
\quad(77) \\
\end{array}$ & $\begin{array}{l}0.23 \\
4.3 \sim 0 \\
\quad(96) \\
\end{array}$ & $\begin{array}{l}0.09 \\
4.7 \sim 0 \\
\quad(84)\end{array}$ & $\begin{array}{l}0.07 \\
3.2 \sim 0 \\
(74) \\
\end{array}$ & $\begin{array}{l}0.18 \\
4.7 \sim 0 \\
\quad(56)\end{array}$ & $\begin{array}{l}0.01 \\
0.2 \sim 0 \\
\quad(23) \\
\end{array}$ & $\begin{array}{l}0 \\
0\end{array}$ \\
\hline 0.11 & 0 & 0.09 & 0 & 0 & 0 & 0 & 0 \\
\hline $16.5 \sim 0$ & 0 & $11.4 \sim 0$ & 0 & 0 & $0.2 \sim 0$ & 0 & $0.1 \sim 0$ \\
\hline 0.10 & 0.01 & 0 & 0 & 0 & 0 & 0 & 0 \\
\hline $13.8 \sim 0$ & $0,05 \sim 0$ & 0 & $0.5 \sim 0$ & $0.2 \sim 0$ & 0 & $0.2 \sim 0$ & $0.2 \sim 0$ \\
\hline 0.04 & 0.02 & 0.02 & 0.01 & 0.03 & 0.01 & 0.04 & 0.04 \\
\hline $3.0 \sim 0$ & $1.0 \sim 0$ & $1.3 \sim 0$ & $2.3 \sim 0$ & $2.5 \sim 0$ & $0.5 \sim 0$ & $1.1 \sim 0$ & $1.4 \sim 0$ \\
\hline 0.27 & 0.70 & 0.24 & 0.25 & 0.26 & 0.23 & 0.35 & 0.50 \\
\hline $4.1 \sim 0$ & $2.0 \sim 0$ & $2.6 \sim 0$ & $4.1 \sim 0$ & $3.1 \sim 0$ & $1.7 \sim 0$ & $3.2 \sim 0$ & $3.7 \sim 0$ \\
\hline 0.52 & 0.39 & 0.51 & 0.34 & 0.53 & 0.34 & 0.36 & 0.59 \\
\hline $3.0 \sim 0$ & $2.0 \sim 0$ & $5.0 \sim 0$ & $2.0 \sim 0$ & $3.8 \sim 0$ & $1.7 \sim 0$ & $2.0 \sim 0$ & $3.1 \sim 0$ \\
\hline 0.59 & 0.48 & 0.31 & 0.49 & 0.52 & 0.36 & 0.45 & 0.52 \\
\hline $2.8 \sim 0$ & $2.0 \sim 0$ & $4.0 \sim 0$ & $2.6 \sim 0$ & $2.5 \sim 0$ & $1.4 \sim 0$ & $2.0 \sim 0$ & $2.0 \sim 0$ \\
\hline
\end{tabular}


Table VII. Duration

\begin{tabular}{|c|c|c|c|c|c|c|c|}
\hline & & & & & & & Age \\
\hline \multirow{2}{*}{\multicolumn{2}{|c|}{$\frac{\text { Age }}{\text { Sex }}$}} & \multicolumn{2}{|c|}{$15 \sim 19$} & \multicolumn{2}{|c|}{$20 \sim 29$} & \multicolumn{2}{|c|}{$30 \sim 39$} \\
\hline & & $\mathrm{M}$ & $\mathrm{F}$ & $\mathbf{M}$ & $\mathbf{F}$ & $\mathrm{M}$ & $\mathrm{F}$ \\
\hline \multicolumn{2}{|c|}{ Number of Cases } & 61 & 39 & 165 & 109 & 119 & 94 \\
\hline I & $\begin{array}{l}\text { Mn. } \\
\text { Max. Min. }\end{array}$ & $\begin{array}{c}0.011 \\
0.022 \sim 0 \\
(47)\end{array}$ & $\begin{array}{c}0.007 \\
0.019 \sim 0 \\
(21)\end{array}$ & $\begin{array}{c}0.013 \\
0.024 \sim 0 \\
\quad 65) \\
\end{array}$ & $\begin{array}{c}0.008 \\
0.024 \sim 0 \\
(49) \\
\end{array}$ & $\begin{array}{c}0.011 \\
0.049 \sim 0 \\
(62) \\
\end{array}$ & $\begin{array}{c}0.007 \\
0.024 \sim 0 \\
(50)\end{array}$ \\
\hline II & $\begin{array}{l}\text { Mn. } \\
\text { Max. } \sim \operatorname{Min} .\end{array}$ & $\begin{array}{c}0.011 \\
0.024 \sim 0 \\
(40)\end{array}$ & $\begin{array}{c}0.006 \\
0.019 \sim 0 \\
(20)\end{array}$ & $\begin{array}{c}0.012 \\
0.024 \sim 0 \\
(99)\end{array}$ & $\begin{array}{c}0.010 \\
0.024 \sim 0 \\
(64) \\
\end{array}$ & $\begin{array}{c}0.012 \\
0.054 \sim 0 \\
(68)\end{array}$ & $\begin{array}{c}0.014 \\
0.024 \sim 0 \\
(47)\end{array}$ \\
\hline III & $\begin{array}{l}\text { Mn. } \\
\text { Max, } \sim \operatorname{Min} .\end{array}$ & $\begin{array}{c}0.011 \\
0.024 \sim 0 \\
(43)\end{array}$ & $\begin{array}{c}0.012 \\
0.034 \sim 0 \\
(22) \\
\end{array}$ & $\begin{array}{c}0.013 \\
0.074 \sim 0 \\
(88)\end{array}$ & $\begin{array}{c}0.011 \\
0.034 \sim 0 \\
(66)\end{array}$ & $\begin{array}{c}0.015 \\
0.054 \sim 0 \\
(60) \\
\end{array}$ & $\begin{array}{c}0.010 \\
0.034 \sim 0 \\
(50)\end{array}$ \\
\hline${ }_{a} V_{n}$ & $\begin{array}{l}\text { Mn. } \\
\operatorname{Max}, \sim \operatorname{Min} .\end{array}$ & & & & & & \\
\hline${ }_{\mathrm{a}} \mathrm{V}_{\mathrm{L}}$ & $\begin{array}{l}\text { Mn. } \\
\text { Max. } \sim \operatorname{Min} .\end{array}$ & $\begin{array}{c}0.011 \\
0.024 \sim 0 \\
(32)\end{array}$ & $\begin{array}{l}0.007 \\
0.014 \sim 0 \\
(14)\end{array}$ & $\begin{array}{c}0.009 \\
0.024 \sim 0 \\
(85)\end{array}$ & $\begin{array}{c}0.011 \\
0.049 \sim 0 \\
(44) \\
\end{array}$ & $\begin{array}{c}0.015 \\
0.074 \sim 0 \\
(44)\end{array}$ & $\begin{array}{c}0.008 \\
0.029 \sim 0 \\
(53)\end{array}$ \\
\hline$V_{F}$ & $\begin{array}{l}\text { Mn. } \\
\text { Max. Min }\end{array}$ & $\begin{array}{c}0.011 \\
0.024 \sim 0 \\
(36)\end{array}$ & $\begin{array}{c}0.010 \\
0.019 \sim 0 \\
(21)\end{array}$ & $\begin{array}{c}0.014 \\
0.054 \sim 0 \\
(81)\end{array}$ & $\begin{array}{c}0.010 \\
0.029 \sim 0 \\
(56)\end{array}$ & $\begin{array}{c}0.011 \\
0.024 \sim 0 \\
(15)\end{array}$ & $\begin{array}{c}0.016 \\
0.054 \sim 0 \\
(46)\end{array}$ \\
\hline $\mathrm{V}_{4} \mathrm{k}$ & $\begin{array}{l}\text { Mn. } \\
\text { Max. Min. }\end{array}$ & $\begin{array}{c}0 \\
0.01 \sim 0 \\
(5)\end{array}$ & $\begin{array}{c}0.007 \\
0.034 \sim 0 \\
(7)\end{array}$ & $\begin{array}{c}0.016 \\
0.034 \sim 0 \\
(5)\end{array}$ & $\begin{array}{c}0.006 \\
0.024 \sim 0 \\
(9) \\
\end{array}$ & $\begin{array}{c}0 \\
0 \\
(0) \\
\end{array}$ & $\begin{array}{c}0.003 \\
0.012 \sim 0 \\
(13) \\
\end{array}$ \\
\hline$V_{1}$ & $\begin{array}{l}\text { Mn. } \\
\text { Max. Min. }\end{array}$ & $\begin{array}{c}0.003 \\
0.014 \sim 0 \\
(22) \\
\end{array}$ & $\begin{array}{c}0.006 \\
0.034 \sim 0 \\
(7) \\
\end{array}$ & $\begin{array}{c}0.002 \\
0.014 \sim 0 \\
(41)\end{array}$ & $\begin{array}{c}0 \\
0 \\
(0) \\
\end{array}$ & $\begin{array}{c}0.029 \\
0.039 \sim 0 \\
-(2)\end{array}$ & $\begin{array}{c}0.005 \\
0.069 \sim 0 \\
(23) \\
\end{array}$ \\
\hline$V_{2}$ & $\begin{array}{l}\text { Mn. } \\
\text { Max. Min. }\end{array}$ & $\begin{array}{c}0.003 \\
0.014 \sim 0 \\
(23) \\
\end{array}$ & $\begin{array}{c}0.007 \\
0.039 \sim 0 \\
(7)\end{array}$ & $\begin{array}{c}0.003 \\
0.014 \sim 0 \\
\quad(44)\end{array}$ & $\begin{array}{c}0 \\
0 \\
(0) \\
\end{array}$ & $\begin{array}{l}0 \\
0 \\
(0) \\
\end{array}$ & $\begin{array}{c}0 \\
0 \\
(0) \\
\end{array}$ \\
\hline $\mathrm{V}_{3}$ & $\begin{array}{l}\text { Mn. } \\
\text { Max. } \sim \operatorname{Min} .\end{array}$ & $\begin{array}{c}0.003 \\
0.012 \sim 0 \\
\quad(15)\end{array}$ & $\begin{array}{c}0 \\
0 \\
(0) \\
\end{array}$ & $\begin{array}{c}0.003 \\
0.014 \sim 0 \\
(45) \\
\end{array}$ & $\begin{array}{c}0.003 \\
0.010 \sim 0 \\
(15) \\
\end{array}$ & $\begin{array}{c}0 \\
0.010 \sim 0 \\
(2)\end{array}$ & $\begin{array}{c}0 \\
0 \\
(0) \\
\end{array}$ \\
\hline$V_{4}$ & $\begin{array}{l}\text { Mn. } \\
\text { Max } \sim \operatorname{Min} .\end{array}$ & $\begin{array}{c}0.009 \\
0.024 \sim 0 \\
(25)\end{array}$ & $\begin{array}{l}0.006 \\
0.019 \sim 0 \\
(11) \\
\end{array}$ & $\begin{array}{c}0.007 \\
0.019 \sim 0 \\
(73) \\
\end{array}$ & $\begin{array}{c}0.005 \\
0.019 \sim 0 \\
(32)\end{array}$ & $\begin{array}{c}0.011 \\
0.024 \sim 0 \\
(35)\end{array}$ & $\begin{array}{c}0.008 \\
0.024 \sim 0 \\
(29)\end{array}$ \\
\hline$V_{5}$ & $\begin{array}{l}\text { Mn. } \\
\text { Max. Min. }\end{array}$ & $\begin{array}{c}0.014 \\
0.029 \sim 0 \\
(44)\end{array}$ & $\begin{array}{c}0.009 \\
0.019 \sim 0 \\
(17) \\
\end{array}$ & $\begin{array}{c}0.013 \\
0.029 \sim 0 \\
(117) \\
\end{array}$ & $\begin{array}{c}0.011 \\
0.024 \sim 0 \\
(66) \\
\end{array}$ & $\begin{array}{c}0.011 \\
0.024 \sim 0 \\
(74)\end{array}$ & $\begin{array}{c}0.010 \\
0.02 \sim 0 \\
(54)\end{array}$ \\
\hline$V_{0}$ & $\begin{array}{l}\text { Mn. } \\
\text { Max. Min. }\end{array}$ & $\begin{array}{c}0.014 \\
0.029 \sim 0 \\
(46)\end{array}$ & $\begin{array}{c}0.015 \\
0.024 \sim 0 \\
(23)\end{array}$ & $\begin{array}{c}0.014 \\
0.024 \sim 0 \\
(122)\end{array}$ & $\begin{array}{c}0.010 \\
0.029 \sim 0 \\
(10)\end{array}$ & $\begin{array}{c}0.012 \\
0.024 \sim 0 \\
(88)\end{array}$ & $\begin{array}{c}0.011 \\
0.02 \sim 0 \\
(60)\end{array}$ \\
\hline
\end{tabular}

( ): Number of cases in which QI was measurable. 
Vol. 4 NORMAL VALUE OF ELECTROCARDIOGRAM IN THE JAPANESE 155
No. 2 Th of $Q_{\mathbf{r}}$ (in sec.)

Group (yr.)

\begin{tabular}{|c|c|c|c|c|c|c|c|}
\hline \multicolumn{2}{|c|}{$40 \sim 49$} & \multicolumn{2}{|c|}{$50 \sim 59$} & \multicolumn{2}{|c|}{$60 \sim 69$} & \multicolumn{2}{|c|}{$70 \sim$} \\
\hline $\mathbf{M}$ & $F$ & $\mathrm{M}$ & $F$ & $\mathbf{M}$ & $\mathrm{F}$ & $\mathbf{M}$ & $\mathbf{F}$ \\
\hline 153 & 82 & 135 & 102 & 101 & 79 & 48 & 42 \\
\hline $\begin{array}{c}0.006 \\
0.025 \sim 0 \\
(115)\end{array}$ & $\begin{array}{c}0.012 \\
0.024 \sim 0 \\
(30) \\
\end{array}$ & $\begin{array}{c}0.011 \\
0.024 \sim 0 \\
(53)\end{array}$ & $\begin{array}{c}0.009 \\
1.024 \sim 0 \\
\quad(43)\end{array}$ & $\begin{array}{c}0.011 \\
0.024 \sim 0 \\
(42)\end{array}$ & $\begin{array}{c}0.011 \\
0.024 \sim 0 \\
(32)\end{array}$ & $\begin{array}{c}0.010 \\
0.024 \sim 0 \\
(22) \\
\end{array}$ & $\begin{array}{c}0.010 \\
0.019 \sim 0 \\
(25)\end{array}$ \\
\hline $\begin{array}{c}0.015 \\
0.044 \sim 0 \\
(80)\end{array}$ & $\begin{array}{c}0.010 \\
0.019 \sim 0 \\
(41)\end{array}$ & $\begin{array}{c}0.011 \\
0.034 \sim 0 \\
(57)\end{array}$ & $\begin{array}{c}0.009 \\
0.024 \sim 0 \\
(43)\end{array}$ & $\begin{array}{c}0.012 \\
0.024 \sim 0 \\
(38)\end{array}$ & $\begin{array}{c}0.011 \\
0.024 \sim 0 \\
(25)\end{array}$ & $\begin{array}{c}0.012 \\
0.024 \sim 0 \\
\quad(18)\end{array}$ & $\begin{array}{l}0.011 \\
0.024 \sim 0 \\
(20)\end{array}$ \\
\hline $\begin{array}{c}0.014 \\
0.03 \sim 0 \\
(77) \\
\end{array}$ & $\begin{array}{c}0.012 \\
0.034 \sim 0 \\
(38)\end{array}$ & $\begin{array}{c}0.013 \\
0.034 \sim 0 \\
(57)\end{array}$ & $\begin{array}{c}0.011 \\
1.124 \sim 0 \\
\quad(32)\end{array}$ & $\begin{array}{c}0.011 \\
0.027 \sim 0 \\
\quad(34)\end{array}$ & $\begin{array}{c}0.014 \\
0.034 \sim 0 \\
(31)\end{array}$ & $\begin{array}{c}0.012 \\
0.029 \sim 0 \\
\quad(18) \\
\end{array}$ & $\begin{array}{c}0.014 \\
0.024 \sim 0 \\
(10)\end{array}$ \\
\hline $\begin{array}{c}0.013 \\
0.04 \sim 0 \\
(74)\end{array}$ & $\begin{array}{c}0.011 \\
0.024 \sim 0 \\
(33)\end{array}$ & $\begin{array}{c}0.014 \\
0.034 \sim 0 \\
(52)\end{array}$ & $\begin{array}{c}0.011 \\
0.024 \sim 0 \\
-\quad(51)\end{array}$ & $\begin{array}{c}0.014 \\
0.044 \sim 0 \\
(47)\end{array}$ & $\begin{array}{c}0.011 \\
0.044 \sim 0 \\
(31)\end{array}$ & $\begin{array}{c}0.019 \\
0.034 \sim 0 \\
(26)\end{array}$ & $\begin{array}{c}0.016 \\
0.044 \sim 0 \\
(25)\end{array}$ \\
\hline $\begin{array}{c}0.003 \\
0.020 \sim 0 \\
(75)\end{array}$ & $\begin{array}{c}0.010 \\
0.029 \sim 0 \\
(33)\end{array}$ & $\begin{array}{c}0.013 \\
0.029 \sim 0 \\
(47)\end{array}$ & $\begin{array}{c}0.011 \\
0.029 \sim 0 \\
(30)\end{array}$ & $\begin{array}{c}0.011 \\
0.024 \sim 0 \\
(53)\end{array}$ & $\begin{array}{c}0.011 \\
0.034 \sim 0 \\
(26)\end{array}$ & $\begin{array}{c}0.012 \\
0.024 \sim 0 \\
(13)\end{array}$ & $\begin{array}{l}0.013 \\
0.029 \sim 0 \\
(11)\end{array}$ \\
\hline $\begin{array}{c}0.010 \\
0.03 \sim 0 \\
(14)\end{array}$ & $\begin{array}{c}0.009 \\
0.034 \sim 0 \\
(12)\end{array}$ & $\begin{array}{c}0.007 \\
0.014 \sim 0 \\
(3)\end{array}$ & $\begin{array}{c}0.013 \\
0.024 \sim 0 \\
(5)\end{array}$ & $\begin{array}{c}0.014 \\
0.019 \sim 0 \\
(3)\end{array}$ & $\begin{array}{c}0.012 \\
0.019 \sim 0 \\
(3)\end{array}$ & $\begin{array}{c}0 \\
0 \\
(0) \\
\end{array}$ & $\begin{array}{c}0 \\
0 \\
(0) \\
\end{array}$ \\
\hline $\begin{array}{c}0.001 \\
0.03 \sim 0 \\
(33)\end{array}$ & $\begin{array}{l}0 \\
0 \\
(0)\end{array}$ & $\begin{array}{c}0.004 \\
0.004 \sim 0 \\
(1)\end{array}$ & $\begin{array}{c}0.014 \\
0.024 \sim 0 \\
(2)\end{array}$ & $\begin{array}{c}0 \\
0 \\
(0) \\
\end{array}$ & $\begin{array}{c}0.004 \\
0.004 \sim 0 \\
(3)\end{array}$ & $\begin{array}{c}0 \\
0 \\
(0)\end{array}$ & $\begin{array}{c}0.014 \\
0.014 \sim 0 \\
(1)\end{array}$ \\
\hline $\begin{array}{c}0.002 \\
0.02 \sim 0 \\
(31)\end{array}$ & $\begin{array}{c}0 \\
0 \\
(0)\end{array}$ & $\begin{array}{c}0.004 \\
0.004 \sim 0 \\
(1)\end{array}$ & $\begin{array}{c}0.004 \\
0.004 \sim 0 \\
\quad(2) \\
\end{array}$ & $\begin{array}{l}0 \\
0 \\
(0) \\
\end{array}$ & $\begin{array}{c}0.004 \\
0.004 \sim 0 \\
(1)\end{array}$ & $\begin{array}{c}0.019 \\
0.019 \sim 0 \\
(1)\end{array}$ & $\begin{array}{c}0.009 \\
0.009 \sim 0 \\
(1)\end{array}$ \\
\hline $\begin{array}{c}0 \\
0 \\
(0) \\
\end{array}$ & $\begin{array}{c}0.005 \\
0.014 \sim 0 \\
(9)\end{array}$ & $\begin{array}{c}0.011 \\
0.014 \sim 0 \\
(6)\end{array}$ & $\begin{array}{c}0.007 \\
0.009 \sim 0 \\
(5)\end{array}$ & $\begin{array}{c}0.011 \\
0.014 \sim 0 \\
(2)\end{array}$ & $\begin{array}{c}0.009 \\
0.014 \sim 0 \\
(5)\end{array}$ & $\begin{array}{c}0.014 \\
0.019 \sim 0 \\
(2)\end{array}$ & $\begin{array}{c}0.000 \\
0.015 \sim 0 \\
(5)\end{array}$ \\
\hline $\begin{array}{c}0.002 \\
0.015 \sim 0 \\
(37)\end{array}$ & $\begin{array}{c}0.010 \\
0.024 \sim 0 \\
(20)\end{array}$ & $\begin{array}{c}0.010 \\
0.024 \sim 0 \\
(31)\end{array}$ & $\begin{array}{c}0.006 \\
0.014 \sim 0 \\
(18)\end{array}$ & $\begin{array}{c}0.011 \\
0.019 \sim 0 \\
(23)\end{array}$ & $\begin{array}{c}0.011 \\
0.019 \sim 0 \\
(16)\end{array}$ & $\begin{array}{c}0.012 \\
0.024 \sim 0 \\
(14)\end{array}$ & $\begin{array}{c}0.012 \\
0.024 \sim 0 \\
(13)\end{array}$ \\
\hline $\begin{array}{c}0.011 \\
0.025 \sim 0 \\
(86)\end{array}$ & $\begin{array}{c}0.011 \\
0.24 \sim 0 \\
(45)\end{array}$ & $\begin{array}{c}0.011 \\
0.124 \sim 0 \\
(73)\end{array}$ & $\begin{array}{c}0.009 \\
0.024 \sim 0 \\
(55)\end{array}$ & $\begin{array}{c}0.011 \\
0.034 \sim 0 \\
\quad(53)\end{array}$ & $\begin{array}{c}0.011 \\
0.019 \sim 0 \\
(35)\end{array}$ & $\begin{array}{c}0.012 \\
0.024 \sim 0 \\
(28)\end{array}$ & $\begin{array}{c}0.014 \\
0.024 \sim 0 \\
(21)\end{array}$ \\
\hline $\begin{array}{c}0.011 \\
0.025 \sim 0 \\
(104)\end{array}$ & $\begin{array}{c}0.012 \\
0.024 \sim 0 \\
(53)\end{array}$ & $\begin{array}{c}0.015 \\
0.29 \sim 0 \\
(93)\end{array}$ & $\begin{array}{c}0.01 \\
0.024 \sim 0 \\
(69)\end{array}$ & $\begin{array}{l}0.011 \\
0.024 \sim 0 \\
(66)\end{array}$ & $\begin{array}{c}0.011 \\
0.019 \sim 0 \\
(41)\end{array}$ & $\begin{array}{c}0.013 \\
0.024 \sim 0 \\
(37)\end{array}$ & $\begin{array}{c}0.013 \\
0.029 \sim 0 \\
(25)\end{array}$ \\
\hline
\end{tabular}


Table VIII. Duration

\begin{tabular}{|c|c|c|c|c|c|c|c|}
\hline & & & & & & & Age \\
\hline \multirow{2}{*}{\multicolumn{2}{|c|}{$\frac{\text { Age }}{\text { Sex }}$}} & \multicolumn{2}{|c|}{$15 \sim 19$} & \multicolumn{2}{|c|}{$20 \sim 29$} & \multicolumn{2}{|c|}{$30 \sim 39$} \\
\hline & & $\mathbf{M}$ & $F$ & $\mathbf{M}$ & $F$ & $M$ & F \\
\hline \multicolumn{2}{|c|}{ Number of Cases } & 61 & 39 & 165 & 109 & 119 & 94 \\
\hline I & $\begin{array}{l}\text { Mn. } \\
\text { Max. Min. }\end{array}$ & $\begin{array}{c}0.014 \\
0.029 \sim 0 \\
(47)\end{array}$ & $\begin{array}{c}0.010 \\
0.024 \sim 0 \\
(20)\end{array}$ & $\begin{array}{c}0.013 \\
0.029 \sim 0 \\
(67) \\
\end{array}$ & $\begin{array}{c}0.012 \\
0.024 \sim 0 \\
(49) \\
\end{array}$ & $\begin{array}{c}0.027 \\
0.094 \sim 0 \\
\quad(62) \\
\end{array}$ & $\begin{array}{c}0.011 \\
0.029 \sim 0 \\
(50)\end{array}$ \\
\hline II & $\begin{array}{l}\text { Mn. } \\
\text { Max. Min. }\end{array}$ & $\begin{array}{c}0.016 \\
0.044 \sim 0 \\
(40)\end{array}$ & $\begin{array}{c}0.016 \\
0.024 \sim 0 \\
(20) \\
\end{array}$ & $\begin{array}{c}0.017 \\
0.074 \sim 0 \\
(103)\end{array}$ & $\begin{array}{c}0.015 \\
0.034 \sim 0 \\
(64)\end{array}$ & $\begin{array}{c}0.019 \\
0.084 \sim 0 \\
(68) \\
\end{array}$ & $\begin{array}{c}0.013 \\
0.034 \sim 0 \\
(47)\end{array}$ \\
\hline III & $\begin{array}{l}\text { Mn., } \\
\text { Max. Min. }\end{array}$ & $\begin{array}{c}0.018 \\
0.054 \sim 0 \\
(43) \\
\end{array}$ & $\begin{array}{c}0.018 \\
0.039 \sim 0 \\
(22)\end{array}$ & $\begin{array}{c}0.018 \\
0.034 \sim 0 \\
(92)\end{array}$ & $\begin{array}{c}0.018 \\
0.034 \sim 0 \\
(67) \\
\end{array}$ & $\begin{array}{c}0.024 \\
0.084 \sim 0 \\
(59) \\
\end{array}$ & $\begin{array}{c}0.016 \\
0.039 \sim 0 \\
(50) \\
\end{array}$ \\
\hline${ }_{a} V_{R}$ & $\begin{array}{l}\text { Mn. } \\
\text { Max. Min. }\end{array}$ & & & & & & \\
\hline${ }_{\mathrm{a}} \mathrm{V}_{\mathrm{L}}$ & $\begin{array}{l}\text { Mn. } \\
\text { Max. Min. }\end{array}$ & $\begin{array}{c}0.017 \\
0.044 \sim 0 \\
(32) \\
\end{array}$ & $\begin{array}{c}0.012 \\
0.024 \sim 0 \\
(14) \\
\end{array}$ & $\begin{array}{c}0.014 \\
0.054 \sim 0 \\
(87)\end{array}$ & $\begin{array}{c}0.015 \\
0.044 \sim 0 \\
\quad(43) \\
\end{array}$ & $\begin{array}{c}0.027 \\
0.154 \sim 0 \\
(45)\end{array}$ & $\begin{array}{c}0.013 \\
0.054 \sim 0 \\
(52)\end{array}$ \\
\hline${ }_{\mathrm{a}} \mathrm{V}_{\mathrm{F}}$ & $\begin{array}{l}\text { Mn. } \\
\text { Max. Min. }\end{array}$ & $\begin{array}{c}0.017 \\
0.044 \sim 0 \\
\quad(73)\end{array}$ & $\begin{array}{c}0.015 \\
0.024 \sim 0 \\
(20) \\
\end{array}$ & $\begin{array}{c}0.019 \\
0.039 \sim 0 \\
(88) \\
\end{array}$ & $\begin{array}{c}0.015 \\
0.003 \sim 0 \\
(56) \\
\end{array}$ & $\begin{array}{c}0.020 \\
0.044 \sim 0 \\
(52)\end{array}$ & $\begin{array}{c}0.010 \\
0.039 \sim 0 \\
(47)\end{array}$ \\
\hline$V_{4} \mathrm{R}$ & $\begin{array}{l}\text { Mn. } \\
\text { Max. Min. }\end{array}$ & $\begin{array}{c}0 \\
0 \\
-(0) \\
\end{array}$ & $\begin{array}{c}0.014 \\
0.044 \sim 0 \\
(7) \\
\end{array}$ & $\begin{array}{c}0.030 \\
0.060 \sim 0 \\
-\quad(4)\end{array}$ & $\begin{array}{c}0.011 \\
0.034 \sim 0 \\
(9) \\
\end{array}$ & {$\left[\begin{array}{c}0.041 \\
0.064 \sim 0.020 \\
(3) \\
\end{array}\right.$} & $\begin{array}{c}0.004 \\
0.024 \sim 0 \\
(11)\end{array}$ \\
\hline$V_{1}$ & $\begin{array}{l}\text { Mn. } \\
\text { Max. } \sim \operatorname{Min} .\end{array}$ & $\begin{array}{c}0.003 \\
0.014 \sim 0 \\
\quad(22) \\
\end{array}$ & $\begin{array}{c}0.009 \\
0.040 \sim 0 \\
(7) \\
\end{array}$ & $\begin{array}{c}0.005 \\
0.005 \sim 0 \\
(1)\end{array}$ & $\begin{array}{c}0 \\
0 \\
0 \\
\end{array}$ & $\begin{array}{c}0.049 \\
0.069 \sim 0 \\
\quad(2) \\
\end{array}$ & $\begin{array}{c}0.003 \\
0.019 \sim 0 \\
(23)\end{array}$ \\
\hline$v_{2}$ & $\begin{array}{l}\text { Mn. } \\
\text { Max. Min. }\end{array}$ & $\begin{array}{c}0.003 \\
0.024 \sim 0 \\
-(23) \\
\end{array}$ & $\begin{array}{c}0.014 \\
0.054 \sim 0 \\
(7) \\
\end{array}$ & $\begin{array}{c}0.003 \\
0.005 \sim 0 \\
(2)\end{array}$ & $\begin{array}{l}0 \\
0 \\
(0)\end{array}$ & $\begin{array}{c}0.009 \\
0.009 \sim 0 \\
(1) \\
\end{array}$ & $\begin{array}{c}0 \\
0 \\
(0) \\
\end{array}$ \\
\hline $\mathrm{V}_{3}$ & $\begin{array}{l}\text { Mn. } \\
\text { Max. Min, }\end{array}$ & $\begin{array}{c}0.004 \\
0.024 \sim 0 \\
\quad(15)\end{array}$ & $\begin{array}{c}0.004 \\
0.004 \sim 0 \\
-\quad(6) \\
\end{array}$ & $\begin{array}{c}0.007 \\
0.02 \sim 0 \\
(3)\end{array}$ & $\begin{array}{c}0.003 \\
0.014 \sim 0 \\
(15)\end{array}$ & $\begin{array}{c}0.011 \\
0.024 \sim 0 \\
(2)\end{array}$ & $\begin{array}{l}0 \\
0 \\
(0) \\
\end{array}$ \\
\hline $\mathrm{V}_{4}$ & $\begin{array}{l}\text { Mn. } \\
\text { Max. Min. }\end{array}$ & $\begin{array}{c}0.012 \\
0.029 \sim 0 \\
(35)\end{array}$ & $\begin{array}{c}0.011 \\
0.019 \sim 0 \\
(11)\end{array}$ & $\begin{array}{c}0.010 \\
0.034 \sim 0 \\
(76) \\
\end{array}$ & $\begin{array}{c}0.008 \\
0.029 \sim 0 \\
(32)\end{array}$ & $\begin{array}{c}0.018 \\
0.034 \sim 0 \\
(36)\end{array}$ & $\begin{array}{c}0.009 \\
0.034 \sim 0 \\
(29)\end{array}$ \\
\hline $\mathrm{V}_{\mathrm{s}}$ & $\begin{array}{l}\text { Mn. } \\
\text { Max. Min. }\end{array}$ & $\begin{array}{c}0.018 \\
0.044 \sim 0 \\
\quad(46) \\
\end{array}$ & $\begin{array}{c}0.012 \\
0.024 \sim 0 \\
\quad(18)\end{array}$ & $\begin{array}{c}0.017 \\
0.034 \sim 0 \\
(119)\end{array}$ & $\begin{array}{c}0.014 \\
0.044 \sim 0 \\
(55)\end{array}$ & $\begin{array}{c}0.018 \\
0.034 \sim 0 \\
(77) \\
\end{array}$ & $\begin{array}{c}0.012 \\
0.034 \sim 0 \\
(55)\end{array}$ \\
\hline$V_{6}$ & $\begin{array}{l}\text { Mn. } \\
\text { Max. Min. }\end{array}$ & $\begin{array}{c}0.019 \\
0.044 \sim 0 \\
(48)\end{array}$ & $\begin{array}{c}0.011 \\
0.019 \sim 0 \\
(22)\end{array}$ & $\begin{array}{c}0.021 \\
0.034 \sim 0 \\
(128)\end{array}$ & $\begin{array}{c}0.014 \\
0.044 \sim 0 \\
(70)\end{array}$ & $\begin{array}{c}0.030 \\
0.034 \sim 0 \\
(71)\end{array}$ & $\begin{array}{c}0.015 \\
0.039 \sim 0 \\
(61)\end{array}$ \\
\hline
\end{tabular}




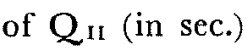

Group (yr.)

\begin{tabular}{|c|c|c|c|c|c|c|c|}
\hline \multicolumn{2}{|c|}{$40 \sim 49$} & \multicolumn{2}{|c|}{$50 \sim 59$} & \multicolumn{2}{|c|}{$60 \sim 69$} & \multicolumn{2}{|c|}{$70 \sim$} \\
\hline M & F & M & F & $\mathbf{M}$ & F & $\mathbf{M}$ & $\mathrm{F}$ \\
\hline 153 & 82 & 135 & 102 & 101 & 79 & 48 & 42 \\
\hline $\begin{array}{c}0.020 \\
0204 \sim 0 \\
(69)\end{array}$ & $\begin{array}{c}0.018 \\
0.049 \sim 0 \\
(30)\end{array}$ & $\begin{array}{c}0.016 \\
0.044 \sim 0 \\
(53)\end{array}$ & $\begin{array}{c}0.014 \\
0.044 \sim 0 \\
(45)\end{array}$ & $\begin{array}{c}0.015 \\
0.029 \sim 0 \\
(44)\end{array}$ & $\begin{array}{c}0.016 \\
0.034 \sim 0 \\
(33)\end{array}$ & $\begin{array}{c}1.018 \\
0.034 \sim 0 \\
(23)\end{array}$ & $\begin{array}{c}0.015 \\
0.029 \sim 0 \\
(26) \\
\end{array}$ \\
\hline $\begin{array}{c}0.018 \\
0.304 \sim 0 \\
(81)\end{array}$ & $\begin{array}{c}0.014, \\
0.039 \sim 0 \\
(41)\end{array}$ & $\begin{array}{c}0.017 \\
0.044 \sim 0 \\
\quad(58)\end{array}$ & $\begin{array}{c}0.013 \\
0.034 \sim 0 \\
\quad(43)\end{array}$ & $\begin{array}{c}0.016 \\
0.029 \sim 0 \\
(37) \\
\end{array}$ & $\begin{array}{c}0.017 \\
0.034 \sim 0 \\
(25)\end{array}$ & $\begin{array}{c}0.018 \\
0.034 \sim 0 \\
(19)\end{array}$ & $\begin{array}{c}0.017 \\
0.044 \sim 0 \\
(20)\end{array}$ \\
\hline $\begin{array}{c}0.018 \\
0.204 \sim 0 \\
(78)\end{array}$ & $\begin{array}{c}0.019 \\
0.039 \sim 0 \\
(38)\end{array}$ & $\begin{array}{c}0.020 \\
0.044 \sim 0 \\
\quad(56)\end{array}$ & $\begin{array}{c}0.019 \\
0.044 \sim 0 \\
(32)\end{array}$ & $\begin{array}{c}0.018 \\
0.054 \sim 0 \\
(36)\end{array}$ & $\begin{array}{c}0.023 \\
0.044 \sim 0 \\
(31)\end{array}$ & $\begin{array}{c}0.018 \\
0.034 \sim 0 \\
(15)\end{array}$ & $\begin{array}{c}0.022 \\
0.044 \sim 0 \\
\quad(10)\end{array}$ \\
\hline $\begin{array}{c}0.027 \\
0.060 \sim 0 \\
(77)\end{array}$ & $\begin{array}{c}0.017 \\
0.054 \sim 0 \\
(34)\end{array}$ & $\begin{array}{c}0.025 \\
0.084 \sim 0 \\
(53)\end{array}$ & $\begin{array}{c}0.017, \\
0.034 \sim 0 \\
(46)\end{array}$ & $\begin{array}{r}0.021 \\
0.054 \sim \\
\quad(46)\end{array}$ & $\begin{array}{c}0.021 \\
0.084 \sim 0 \\
(31)\end{array}$ & $\begin{array}{c}0.029 \\
0.054 \sim 0 \\
(26)\end{array}$ & $\begin{array}{c}0.023 \\
0.074 \sim 0 \\
(26)\end{array}$ \\
\hline $\begin{array}{c}0.012 \\
0.039 \sim 0 \\
(77) \\
\end{array}$ & $\begin{array}{c}0.018 \\
0.054 \sim 0 \\
(33)\end{array}$ & $\begin{array}{c}0.020 \\
0.044 \sim 0 \\
(47)\end{array}$ & $\begin{array}{c}0.018 \\
0.044 \sim 0 \\
(29)\end{array}$ & $\begin{array}{c}0.016 \\
0.029 \sim 0 \\
(36)\end{array}$ & $\begin{array}{c}0.017 \\
0.034 \sim 0 \\
(26)\end{array}$ & $\begin{array}{c}0.017 \\
0.034 \sim 0 \\
(14) \\
\end{array}$ & $\begin{array}{c}0.020 \\
0.044 \sim 0 \\
(12)\end{array}$ \\
\hline $\begin{array}{c}0.017 \\
0.084 \sim 0 \\
(15)\end{array}$ & $\begin{array}{c}0.014 \\
0.054 \sim 0 \\
(12)\end{array}$ & $\begin{array}{c}0.010 \\
0.019 \sim 0 \\
(3)\end{array}$ & $\begin{array}{c}0.044 \sim 0 \\
(5)\end{array}$ & $\begin{array}{c}\mathbf{0 . 0 0 1} \\
0.064 \sim 0 \\
(3) \\
\end{array}$ & $\begin{array}{c}0.022 \\
0.039 \sim 0 \\
(3)\end{array}$ & $\begin{array}{l}0 \\
0 \\
(0) \\
\end{array}$ & $\begin{array}{c}0 \\
0 \\
0\end{array}$ \\
\hline $\begin{array}{c}0.005 \\
0.044 \sim 0 \\
(31)\end{array}$ & $\begin{array}{l}0 \\
0 \\
(0) \\
\end{array}$ & $\begin{array}{c}0.007 \\
0.014 \sim 0 \\
\quad(2)\end{array}$ & $\begin{array}{c}0.000 \\
0.039 \sim 0 \\
(2)\end{array}$ & $\begin{array}{c}0 \\
0 \\
(0) \\
\end{array}$ & $\begin{array}{c}0 \\
0 \\
0 \\
\end{array}$ & $\begin{array}{c}0 \\
0 \\
(0) \\
\end{array}$ & $\begin{array}{c}0.010 \\
0.010 \sim 0 \\
(1)\end{array}$ \\
\hline $\begin{array}{c}0.005 \\
0.044 \sim 0 \\
(30) \\
\end{array}$ & $\begin{array}{c}0 \\
0 \\
(0)\end{array}$ & $\begin{array}{c}0.004 \\
0.004 \sim 0 \\
(1)\end{array}$ & $\begin{array}{c}0.003 \\
0.009 \sim 0 \\
(2)\end{array}$ & $\begin{array}{c}0 \\
0 \\
(0) \\
\end{array}$ & $\begin{array}{c}0 \\
0 \\
(0) \\
\end{array}$ & $\begin{array}{c}0.024 \\
0.024 \sim 0 \\
(1)\end{array}$ & $\begin{array}{c}0.009 \\
0.009 \sim 0 \\
(1)\end{array}$ \\
\hline $\begin{array}{c}0 \\
0 \\
(0) \\
\end{array}$ & $\begin{array}{c}0.001 \\
0.054 \sim 0 \\
(3)\end{array}$ & $\begin{array}{c}0.004 \\
0.024 \sim 0 \\
(5)\end{array}$ & $\begin{array}{c}0.007 \\
0.019 \sim 0 \\
(4)\end{array}$ & $\begin{array}{c}0.014 \\
0.019 \sim 0 \\
(2)\end{array}$ & $\begin{array}{r}0 \\
0 \\
0 \\
\end{array}$ & $\begin{array}{c}0.019 \\
0.024 \sim 0 \\
(2)\end{array}$ & $\begin{array}{c}0.002, \\
0.024 \sim 0 \\
\quad(5)\end{array}$ \\
\hline $\begin{array}{c}0.013 \\
0.300 \sim 0 \\
(35)\end{array}$ & $\begin{array}{c}0.013 \\
0.029 \sim 0 \\
(14)\end{array}$ & $\begin{array}{c}0.014 \\
0.044 \sim 0 \\
\quad(30)\end{array}$ & $\begin{array}{c}0.013 \\
0.024 \sim 0 \\
\quad(18)\end{array}$ & $\begin{array}{c}0.014 \\
0.029 \sim 0 \\
(24)\end{array}$ & $\begin{array}{c}0.012 \\
0.029 \sim 0 \\
\quad(16) \\
\end{array}$ & $\begin{array}{c}0.018 \\
0.034 \sim 0 \\
(14)\end{array}$ & $\begin{array}{c}0.020 \\
0.034 \sim 0 \\
(13) \\
\end{array}$ \\
\hline $\begin{array}{c}0.017 \\
0.044 \sim 0 \\
(87)\end{array}$ & $\begin{array}{c}0.017 \\
0.044 \sim 0 \\
\quad(43)\end{array}$ & $\begin{array}{c}0.016 \\
0.049 \sim 0 \\
(75)\end{array}$ & $\begin{array}{c}0.014, \\
0.044 \sim 0 \\
(57)\end{array}$ & $\begin{array}{c}0.016 \\
0.039 \sim 0 \\
(54)\end{array}$ & $\begin{array}{c}0.014 \\
0.029 \sim 0 \\
(35)\end{array}$ & $\begin{array}{c}0.018 \\
0.034 \sim 0 \\
(30)\end{array}$ & $\begin{array}{c}0.019 \\
0.039 \sim 0 \\
(21)\end{array}$ \\
\hline $\begin{array}{l}0.025, \\
0.044 \sim 0 \\
(108)\end{array}$ & $\begin{array}{c}0.019 \\
0.054 \sim 0 \\
(56)\end{array}$ & $\begin{array}{c}0.018 \\
0.044 \sim 0 \\
(94)\end{array}$ & $\begin{array}{c}0.015 \\
0.034 \sim 0 \\
(62)\end{array}$ & $\begin{array}{l}0.017 \\
0.034 \sim 0 \\
(66)\end{array}$ & $\begin{array}{c}0.015 \\
0.029 \sim 0 \\
(42)\end{array}$ & $\begin{array}{c}0.018 \\
0.034 \sim 0 \\
(37)\end{array}$ & $\begin{array}{c}0.019 \\
0.039 \sim 0 \\
(25)\end{array}$ \\
\hline
\end{tabular}


Table IX. Voltage of

\begin{tabular}{|c|c|c|c|c|c|c|c|}
\hline & Age & \multicolumn{2}{|c|}{$15 \sim 19$} & \multicolumn{2}{|c|}{$20 \sim 29$} & \multicolumn{2}{|c|}{$30 \sim 39$} \\
\hline & Sex & & $\mathrm{F}$ & $\mathrm{M}$ & $\mathrm{F}$ & $\mathbf{M}$ & $\mathbf{F}$ \\
\hline Num & per of Cases & 61 & 39 & 165 & 109 & 119 & 94 \\
\hline I & $\begin{array}{l}\text { Mn., SD } \\
\text { Max. } \sim \operatorname{Min} .\end{array}$ & $\begin{array}{l}5.68,2.34 \\
13.0 \sim 2.0\end{array}$ & $\begin{array}{l}5.41,2.13 \\
10.0 \sim 2.2\end{array}$ & $\begin{array}{l}5.14,2.67 \\
15.3 \sim 0.26\end{array}$ & $\begin{array}{l}5.56,2.59 \\
15.3 \sim 0.43\end{array}$ & $\begin{array}{l}5.46,2.38 \\
12.9 \sim 1.0\end{array}$ & $\begin{array}{l}5.38,2.38 \\
14.2 \sim 1.0\end{array}$ \\
\hline II & $\begin{array}{l}\text { Mn., SD } \\
\text { Max. } \sim \text { Min. }\end{array}$ & $\begin{array}{l}14.25,4.65 \\
22.1 \sim 6.0\end{array}$ & $\begin{array}{l}11.67,2.99 \\
17.5 \sim 6.5\end{array}$ & $\begin{array}{l}13.73,4.69 \\
2.38 \sim 4.2\end{array}$ & $\begin{array}{l}11.64,3.97 \\
22.7 \sim 2.0\end{array}$ & $\begin{array}{l}12.99,3.82 \\
23.9 \sim 3.0\end{array}$ & $\begin{array}{l}15.35,3.16 \\
19.1 \sim 3.33 \\
\end{array}$ \\
\hline III & $\begin{array}{l}\text { Mn., SD } \\
\text { Max. } \sim \text { Min. }\end{array}$ & $\begin{array}{l}10.0,2.97 \\
23.1 \sim 25\end{array}$ & $\begin{array}{l}7.39,3.95 \\
15.7 \sim 0.2\end{array}$ & $\begin{array}{l}9.44,4.86 \\
22.1 \sim 0.1\end{array}$ & $\begin{array}{c}7.44,4.26 \\
21.5 \sim 0\end{array}$ & $\begin{array}{c}8.15,4.19 \\
19.9 \sim 0\end{array}$ & $\begin{array}{l}5.36,3.34 \\
15.3 \sim 0.4 \\
\end{array}$ \\
\hline${ }_{a} V_{R}$ & $\begin{array}{l}\text { Mn., SD } \\
\text { Max. } \sim \text { Min. }\end{array}$ & $\begin{array}{c}0.48,0.24 \\
3.69 \sim 0.02\end{array}$ & $\begin{array}{c}0.32,0.73 \\
1.5 \sim 0\end{array}$ & $\begin{array}{c}0.49,0.56 \\
3.2 \sim 0\end{array}$ & $\begin{array}{c}0.63,0.74 \\
3.2 \sim 0\end{array}$ & $\begin{array}{c}0.45,0.76 \\
4.9 \sim 0\end{array}$ & $\begin{array}{c}0.50,0.72 \\
2.4 \sim 0\end{array}$ \\
\hline${ }_{i 1} V_{L}$ & $\begin{array}{l}\text { Mn., SD } \\
\text { Max. } \sim \text { Min. }\end{array}$ & $\begin{array}{l}1.62,1.17 \\
6.44 \sim 0.1\end{array}$ & $\begin{array}{c}3.06,1.69 \\
6.9 \sim 0\end{array}$ & $\begin{array}{c}1.93,2.21 \\
16.2 \sim 0\end{array}$ & $\begin{array}{c}1.71,2.09 \\
13.2 \sim 0\end{array}$ & $\begin{array}{c}1.89,1.48 \\
11.9 \sim 0\end{array}$ & $\begin{array}{c}2.21,2.01 \\
9.1 \sim 0 \\
\end{array}$ \\
\hline${ }_{a} V_{F}$ & $\begin{array}{l}\text { Mn., SD } \\
\text { Max. } \sim \operatorname{Min} .\end{array}$ & $\mid \begin{array}{c}11.80,3.53 \\
21.8 \sim 1.2\end{array}$ & $\begin{array}{l}9.55,3.70 \\
17.5 \sim 3.0\end{array}$ & $\begin{array}{l}11.07,4.76 \\
25.9 \sim 0.5\end{array}$ & $\begin{array}{l}10.00,3.88 \\
20.9 \sim 0.4\end{array}$ & $\begin{array}{c}9.57,3.93 \\
19.9 \sim 0\end{array}$ & $\begin{array}{l}7.45,4.71 \\
15.4 \sim 0.2 \\
\end{array}$ \\
\hline$V_{4} R$ & $\begin{array}{l}\text { Mn., SD } \\
\text { Max. } \sim \text { Min. }\end{array}$ & $\begin{array}{c}2.16,3.26 \\
6.2 \sim 0.1 \\
(52)\end{array}$ & $\begin{array}{c}1.43,1.04 \\
3.3 \sim 0 \\
(34)\end{array}$ & $\begin{array}{c}2.42,1.65 \\
13.6 \sim 0 \\
(117)\end{array}$ & $\begin{array}{c}1.51,1.02 \\
18.0 \sim 0 \\
(97) \\
\end{array}$ & $\begin{array}{c}1.80,1.05 \\
16.9 \sim 0 \\
(86) \\
\end{array}$ & $\begin{array}{c}0.98,0.75 \\
3.2 \sim 0.1 \\
(76)\end{array}$ \\
\hline$V_{1}$ & $\begin{array}{l}\text { Mn., SD } \\
\text { Max. } \sim \text { Min. }\end{array}$ & $\begin{array}{l}5.54,2.56 \\
15.5 \sim 1.6\end{array}$ & $\begin{array}{c}3.95,2.44 \\
10.8 \sim 0\end{array}$ & $\begin{array}{l}5.05,3.19 \\
15.8 \sim 0.54\end{array}$ & $\begin{array}{c}3.53,2.55 \\
10.0 \sim 0.23\end{array}$ & $\begin{array}{c}3.67,2.06 \\
10.9 \sim 0\end{array}$ & $\begin{array}{c}2.81,1.66 \\
7.5 \sim 0.2\end{array}$ \\
\hline $\mathrm{V}_{2}$ & $\begin{array}{l}\text { Mn., SD } \\
\text { Max. } \sim \text { Min. }\end{array}$ & $\begin{array}{c}10.78,4.16 \\
15.7 \sim 3.5\end{array}$ & $\begin{array}{c}7.32,3.62 \\
18.0 \sim 0\end{array}$ & $\begin{array}{l}9.76,4.69 \\
22.4 \sim 0.8\end{array}$ & $\begin{array}{l}7.10,3.36 \\
23.7 \sim 1.0\end{array}$ & $\begin{array}{c}7.66,3.95 \\
17.9 \sim 0\end{array}$ & $\begin{array}{l}6.48,3.26 \\
12.5-1.2\end{array}$ \\
\hline$V_{3}$ & $\begin{array}{l}\text { Mn., SD } \\
\text { Max. } \sim \text { Min. }\end{array}$ & $\begin{array}{l}13.66,5.80 \\
33.3 \sim 3.1\end{array}$ & $\begin{array}{l}9.05,3.22 \\
18.5-0.2\end{array}$ & $\begin{array}{l}11.56,5.73 \\
3.70 \sim 1.17\end{array}$ & $\begin{array}{l}9.83,4.50 \\
24.6 \sim 3.5\end{array}$ & $\mid \begin{array}{c}10.10,5.37 \\
29.9 \sim 0\end{array}$ & $\begin{array}{l}4.04,5.34 \\
26.1 \sim 0.4\end{array}$ \\
\hline$V_{1}$ & $\begin{array}{l}\text { Mn., SD } \\
\text { Max. } \sim \text { Min. }\end{array}$ & $\left|\begin{array}{c}20.67,7.05 \\
35.8 \sim 2.3\end{array}\right|$ & $\begin{array}{l}13.49,4.90 \\
24.8 \sim 6.8\end{array}$ & $\begin{array}{l}24.68,7.53 \\
53.2 \sim 5.0\end{array}$ & $\begin{array}{l}14.60,4.90 \\
30.6 \sim 2.87\end{array}$ & $\mid \begin{array}{l}17.49,7.35 \\
41.9 \sim 2.0\end{array}$ & $\begin{array}{l}14.65,5.16 \\
34.0 \sim 7.1\end{array}$ \\
\hline$V_{5}$ & $\begin{array}{l}\text { Mn., SD } \\
\text { Max. } \sim \text { Min. }\end{array}$ & $\begin{array}{l}18.87,5.31 \\
55.0 \sim 8.0\end{array}$ & $\begin{array}{c}13.51,3.44 \\
24.0 \sim 9.0\end{array}$ & $\begin{array}{l}17.45,5.84 \\
43.0 \sim 3.98\end{array}$ & $\begin{array}{l}13.4,4.46 \\
27.4 \sim 2.01\end{array}$ & $\mid \begin{array}{c}15.85 \sim 6.29 \\
33.9 \sim 2.0\end{array}$ & $\begin{array}{l}14.10,4.48 \\
26.4 \sim 1.7\end{array}$ \\
\hline$V_{6}$ & $\begin{array}{l}\text { Mn., SD } \\
\text { Max. } \sim \text { Min. }\end{array}$ & $\begin{array}{l}14.31,4.31 \\
28.2 \sim 7.0\end{array}$ & $\begin{array}{l}11.56,3.82 \\
21.8 \sim 7.0\end{array}$ & $\begin{array}{l}13.54,5.11 \\
29.0 \sim 2.4\end{array}$ & $\left|\begin{array}{l}11.07,3.56 \\
23.7 \sim 1.2\end{array}\right|$ & $\left|\begin{array}{c}11.81,4.54 \\
23.9 \sim 2.0\end{array}\right|$ & $\left\{\begin{array}{l}11.65,3.72 \\
24.9 \sim 1.1\end{array}\right.$ \\
\hline
\end{tabular}


$\mathrm{R}$ Deflection (in $\mathrm{mm}$.)

Group (yr.)

\begin{tabular}{|c|c|c|c|c|c|c|c|}
\hline \multicolumn{2}{|c|}{$40 \sim 49$} & \multicolumn{2}{|c|}{$50 \sim 59$} & \multicolumn{2}{|c|}{$60 \sim 69$} & \multicolumn{2}{|c|}{$70 \sim$} \\
\hline M & $\mathrm{F}$ & M & $\mathrm{F}$ & M & $\mathrm{F}$ & M & $\mathrm{F}$ \\
\hline 153 & 82 & 135 & 102 & 101 & 79 & 48 & 42 \\
\hline $5.56,2.70$ & $5.71,2.87$ & $5.30,2.36$ & $6.46,2.75$ & $5.27,2.98$ & $6.34,25.1$ & $5.05,2.62$ & $6.55,3.18$ \\
\hline $14.6 \sim 0.3$ & $14.3 \sim 0$ & $15.9 \sim 0$ & $14.9 \sim 0.0$ & $16.9 \sim 0$ & $12.9 \sim 0$ & $16.9 \sim 1.0$ & $15.9 \sim 1.0$ \\
\hline $10.74,4.20$ & $9.91,3.66$ & $10.78,4.11$ & $9.17,3.24$ & $9.35,3.98$ & $7.44,4.06$ & $7.89,3.23$ & $7.28,2.98$ \\
\hline $21.0 \sim 0.2$ & $17.0 \sim 0$ & $25.9 \sim 3.0$ & $17.9 \sim 0.0$ & $18.9 \sim 1.0$ & $33.4 \sim 0$ & $15.9 \sim 2.0$ & $16.9 \sim 2.0$ \\
\hline $6.24,4.20$ & $5.16,4.15$ & $6.53,4.36$ & $4.42,3.15$ & $5.51,3.16$ & $3.34,2.66$ & $6.62,3.39$ & $3.18,2.41$ \\
\hline $17.5 \sim 0.3$ & $17.0 \sim 0.5$ & $21.9 \sim 0.0$ & $14.9 \sim 0.0$ & $14.9 \sim 0$ & $12.4 \sim 0$ & $12.4 \sim 0$ & $11.9 \sim 0.0$ \\
\hline $0.74,0.00$ & $0.19,0.00$ & $0.36,0.17$ & $0.35,0.54$ & $0.37,0.24$ & $0.32,0.43$ & $0.30,0.48$ & $0.43,0.48$ \\
\hline $11.9 \sim 0$ & $1.4 \sim 0$ & $2.4 \sim 0$ & $1.9 \sim 0.0$ & $2.4 \sim 0$ & $1.9 \sim 0$ & $1.4 \sim 0$ & $1.9 \sim 0.0$ \\
\hline $2.37,2.52$ & $2.29,2.09$ & $2.16,2.42$ & $3.15,2.02$ & $2.53,2.24$ & $3.29,2.28$ & $3.22,2.28$ & $3.86,0.63$ \\
\hline $14.9 \sim 0.1$ & $10.5 \sim 0.1$ & $12.9 \sim 0$ & $9.9 \sim 0$ & $8.9 \sim 0$ & $11.9 \sim 0$ & $14.9 \sim 0$ & $13.9 \sim 0.0$ \\
\hline $8.01,4.25$ & $7.47,4.71$ & $8.19,4.34$ & $6.52,3.36$ & $6.84,4.81$ & $4.76,2.68$ & $5.97,3.32$ & $4.74,2.30$ \\
\hline $17.2 \sim 0.4$ & $16.5 \sim 0.49$ & $22.9 \sim 0.0$ & $16.9 \sim 0.0$ & $15.9 \sim 0$ & $13.9 \sim 0$ & $13.0 \sim 0$ & $15.9 \sim 0.0$ \\
\hline $\begin{array}{c}1.61,0.00 \\
8.4 \sim 0.2 \\
(106) \\
\end{array}$ & $\begin{array}{c}5.50,0.00 \\
2.8 \sim 0.1 \\
(76) \\
\end{array}$ & $\begin{array}{c}1.31,0.74 \\
4.9 \sim 0 \\
(97)\end{array}$ & $\begin{array}{c}1.04,0.73 \\
4.9 \sim 0.0 \\
(80) \\
\end{array}$ & $\begin{array}{c}1.30,0.96 \\
4.9 \sim 0 \\
(74) \\
\end{array}$ & $\begin{array}{c}1.09,0.40 \\
4.4 \sim 0\end{array}$ & $\begin{array}{c}0.97 \\
2.4 \sim 0\end{array}$ & $\begin{array}{c}1.27,0.85 \\
3.9 \sim 0.0 \\
(17)\end{array}$ \\
\hline $3.17,3.64$ & $2.44,1.62$ & $3.40,2.28$ & $2.59,1.67$ & $3.22,2.57$ & $2.35,1.89$ & $2.14,1.57$ & $2.57,1.47$ \\
\hline $13.9 \sim 0.2$ & $6.1 \sim 0.3$ & $13.9 \sim 0.0$ & $8.9 \sim 0.0$ & $13.9 \sim 0$ & $9.9 \sim 0$ & $8.9 \sim 0$ & $8.9 \sim 0$ \\
\hline $7.0,4.12$ & $5.52,3.20$ & $7.41,4.27$ & $5.95,3.62$ & $6.32,3.38$ & $6.38,3.87$ & $5.74,3.87$ & $6.28,3.43$ \\
\hline $18.6 \sim 1.0$ & $17.2 \sim 0.7$ & $21.9 \sim 0.0$ & $15.9 \sim 0.0$ & $17.9 \sim 0$ & $23.9 \sim 0$ & $14.4 \sim 0$ & $13.9 \sim 0.0$ \\
\hline $9.74,5.38$ & $9.33,5.66$ & $11.13,3.25$ & $9.95,5.77$ & $10.75,5.69$ & $11.65,4.98$ & $11.58,7.40$ & $13.43,6.10$ \\
\hline $24.3 \sim 0.6$ & $27.0 \sim 1.3$ & $29.9 \sim 0.0$ & $35.9 \sim 0.0$ & $27.9 \sim 0$ & $21.9 \sim 0$ & $35.9 \sim 0$ & $31.9 \sim 2.0$ \\
\hline $9.31,6.51$ & $15.86,5.92$ & $17.48,6.04$ & $15.99,5.72$ & $17.50,8.41$ & $17.29,5.37$ & $19.87,7.94$ & $21.09,7.78$ \\
\hline $33.8 \sim 3.4$ & $26.8 \sim 5.5$ & $35.9 \sim 0.0$ & $37.9 \sim 0.0$ & $49.9 \sim 2.0$ & $37.4 \sim 2.0$ & $37.9 \sim 0$ & $39.9 \sim 6.0$ \\
\hline $16.45,6.09$ & $15.50,5.0$ & $16.2,5.52$ & $15.20,5.29$ & $16.87,8.23$ & $14.43,4.88$ & $16.74,5.81$ & $17.33,6.05$ \\
\hline $35.2 \sim 1.8$ & $28.5 \sim 5.6$ & $35.9 \sim 2.0$ & $35.9 \sim 0.0$ & $55.9 \sim 4.0$ & $24.4 \sim 0$ & $24.4 \sim 0$ & $35.9 \sim 6.0$ \\
\hline $13.00,5.06$ & $11.88,4.97$ & $12.20,4.27$ & $11.85,4.44$ & $12.25,5.99$ & $10.30,4.22$ & $11.28,4.05$ & $10.0,3.45$ \\
\hline $24.7 \sim 0.9$ & $26.1 \sim 1.7$ & $25.9 \sim 2.0$ & $23.9 \sim 0$ & $37.9 \sim 2.0$ & $23.9 \sim 0$ & $23.9 \sim 2.0$ & $17.9 \sim 4.0$ \\
\hline
\end{tabular}


Table X. Voltage of

\begin{tabular}{|c|c|c|c|c|c|c|c|}
\hline & \multirow{3}{*}{$\frac{\text { Age }}{\text { Sex }}$} & & & & & & Age \\
\hline & & \multicolumn{2}{|c|}{$15 \sim 19$} & \multicolumn{2}{|c|}{$20 \sim 29$} & \multicolumn{2}{|c|}{$30 \sim 39$} \\
\hline & & $\mathbf{M}$ & $\mathbf{F}$ & $\mathbf{M}$ & $\mathbf{F}$ & $\mathbf{M}$ & $\mathbf{F}$ \\
\hline \multicolumn{2}{|c|}{ Number of Cases } & 61 & 39 & 165 & 109 & 119 & 94 \\
\hline $\mathbf{I}$ & $\begin{array}{l}\text { Mn. } \\
\text { Max. Min. }\end{array}$ & $\begin{array}{l}1.40 \\
5.7 \sim 0\end{array}$ & $\begin{array}{l}0.54 \\
3.4 \sim 0\end{array}$ & $\begin{array}{l}1.19 \\
5.35 \sim 0\end{array}$ & $\begin{array}{l}0.10 \\
4.0 \sim 0\end{array}$ & $\begin{array}{l}0.86 \\
3.9 \sim 0\end{array}$ & $\begin{array}{l}0.63 \\
2.7 \sim 0\end{array}$ \\
\hline II & $\begin{array}{l}\text { Mn. } \\
\text { Max. Min. }\end{array}$ & $\begin{array}{l}2.02 \\
5.8 \sim 0\end{array}$ & $\begin{array}{l}0.76 \\
2.9 \sim 0\end{array}$ & $\begin{array}{l}1.31 \\
6.2 \sim 0\end{array}$ & $\begin{array}{l}0.66 \\
6.2 \sim 0\end{array}$ & $\begin{array}{l}1.30 \\
6.9 \sim 0\end{array}$ & $\begin{array}{l}0.65 \\
4.6 \sim 0\end{array}$ \\
\hline III & $\begin{array}{l}\text { Mn. } \\
\text { Max. Min. }\end{array}$ & $\begin{array}{l}1.08 \\
4.9 \sim 0\end{array}$ & $\begin{array}{l}0.78 \\
4.8 \sim 0\end{array}$ & $\begin{array}{l}0.94 \\
8.9 \sim 0\end{array}$ & $\begin{array}{l}0.53 \\
6.2 \sim 0\end{array}$ & $\begin{array}{c}1.22 \\
11.9 \sim 0\end{array}$ & $\begin{array}{l}0.77 \\
4.1 \sim 0\end{array}$ \\
\hline${ }_{a} V_{R}$ & $\begin{array}{l}\text { Mn. } \\
\text { Max. Min. }\end{array}$ & $\begin{array}{l}9.60 \\
14.4 \sim 0\end{array}$ & $\begin{array}{c}8.83 \\
11.2 \sim 5.6\end{array}$ & $\begin{array}{c}9.05 \\
21.7 \sim 0.17\end{array}$ & $\begin{array}{l}8.92 \\
21.0 \sim 4.0\end{array}$ & $\begin{array}{c}5.87 \\
15.9 \sim 0\end{array}$ & $\begin{array}{l}7.36 \\
11.6 \sim 3.6\end{array}$ \\
\hline${ }_{a} V_{L}$ & $\begin{array}{l}\text { Mn. } \\
\text { Max. Min. }\end{array}$ & $\begin{array}{l}3.27 \\
9.5 \sim 0\end{array}$ & $\begin{array}{l}1.82 \\
7.1 \sim 0\end{array}$ & $\begin{array}{l}2.98 \\
14.2 \sim 0\end{array}$ & $\begin{array}{c}2.12 \\
12.0 \sim 0\end{array}$ & $\begin{array}{c}2.39 \\
15.9 \sim 0\end{array}$ & $\begin{array}{l}1.43 \\
7.2 \sim 0\end{array}$ \\
\hline $\mathrm{a} \mathrm{V}_{\mathrm{F}}$ & $\begin{array}{l}\text { Mn. } \\
\text { Max, Min. }\end{array}$ & $\begin{array}{l}1.42 \\
5.84 \sim 0\end{array}$ & $\begin{array}{l}0.62 \\
2.2 \sim 0\end{array}$ & $\begin{array}{l}1.20 \\
12.0 \sim 0\end{array}$ & $\begin{array}{l}0.53 \\
3.2 \sim 0\end{array}$ & $\begin{array}{c}1.27 \\
12.9 \sim 0\end{array}$ & $\begin{array}{l}0.53 \\
2.0 \sim 0\end{array}$ \\
\hline$V_{4} R$ & $\begin{array}{l}\text { Mn. } \\
\text { Max, Min. }\end{array}$ & $\begin{array}{l}2.99 \\
8.5 \sim 0 \\
(49)\end{array}$ & $\begin{array}{l}3.3 \\
8.4 \sim 0\end{array}$ & $\begin{array}{c}3.99 \\
11.0 \sim 0 \\
\end{array}$ & $\begin{array}{c}2.73 \\
14.0 \sim 0 \\
\end{array}$ & $\begin{array}{l}3.47 \\
8.9 \sim 0\end{array}$ & $\begin{array}{c}3.35 \\
10.2 \sim 0\end{array}$ \\
\hline $\mathrm{V}_{1}$ & $\begin{array}{l}\text { Mn. } \\
\text { Max. Min. }\end{array}$ & $\begin{array}{l}10.85 \\
22.7 \sim 0\end{array}$ & $\begin{array}{c}9.2 \\
22.0 \sim 0\end{array}$ & $\begin{array}{l}9.7 \\
27.4 \sim 0\end{array}$ & $\begin{array}{c}9.59 \\
24.5 \sim 2.3\end{array}$ & $\begin{array}{l}9.71 \\
1.9 \sim 0\end{array}$ & $\begin{array}{c}8.90 \\
15.5 \sim 0\end{array}$ \\
\hline $\mathrm{V}_{2}$ & $\begin{array}{l}\text { Mn. } \\
\text { Max. Min. }\end{array}$ & $\begin{array}{l}18.04 \\
42.5 \sim 0\end{array}$ & $\begin{array}{l}15.8 \\
26.5 \sim 6.5\end{array}$ & $\begin{array}{l}20.05 \\
55.1 \sim 0\end{array}$ & $\begin{array}{l}14.06 \\
34.2 \sim 3.6\end{array}$ & $\begin{array}{l}15.37 \\
37.9 \sim 0\end{array}$ & $\begin{array}{l}13.38 \\
28.8 \sim 2.1\end{array}$ \\
\hline $\mathrm{V}_{3}$ & $\begin{array}{l}\text { Mn. } \\
\text { Max. Min. }\end{array}$ & $\begin{array}{l}13.37 \\
25.0 \sim 0\end{array}$ & $\begin{array}{l}8.8 \\
17.0 \sim 0\end{array}$ & $\begin{array}{l}12.62 \\
34.0 \sim 0\end{array}$ & $\begin{array}{c}7.71 \\
16.0 \sim 0\end{array}$ & $\begin{array}{l}10.26 \\
27.9 \sim 0\end{array}$ & $\begin{array}{c}7.42 \\
16.9 \sim 0\end{array}$ \\
\hline $\mathrm{V}_{4}$ & $\begin{array}{l}\text { Mn. } \\
\text { Max. } \sim \text { Min. }\end{array}$ & $\begin{array}{c}7.22 \\
45.0 \sim 0\end{array}$ & $\begin{array}{c}4.5 \\
11.5 \sim 0\end{array}$ & $\begin{array}{c}6.46 \\
22.6 \sim 0\end{array}$ & $\begin{array}{c}6.42 \\
11.1 \sim 0\end{array}$ & $\begin{array}{c}5.54 \\
23.9 \sim 0\end{array}$ & $\begin{array}{c}4.00 \\
12.3 \sim 0\end{array}$ \\
\hline$V_{5}$ & $\begin{array}{l}\text { Mn. } \\
\text { Max. Min. }\end{array}$ & $\begin{array}{c}2.92 \\
40.0 \sim 0\end{array}$ & $\begin{array}{l}2.07 \\
5.1 \sim 0\end{array}$ & $\begin{array}{l}2.28 \\
10.0 \sim 0\end{array}$ & $\begin{array}{l}1.86 \\
6.4 \sim 0\end{array}$ & $\begin{array}{c}2.02 \\
14.9 \sim 0\end{array}$ & $\begin{array}{l}1.73 \\
5.55 \sim 0\end{array}$ \\
\hline $\mathrm{V}_{6}$ & $\begin{array}{l}\text { Mn. } \\
\text { Max. Min. }\end{array}$ & $\begin{array}{c}1.32 \\
17.8 \sim 0\end{array}$ & $\begin{array}{l}0.62 \\
3.3 \sim 0\end{array}$ & $\begin{array}{c}0.99 \\
10.7 \sim 0\end{array}$ & $\begin{array}{l}0.54 \\
4.5 \sim 0\end{array}$ & $\begin{array}{l}0.85 \\
6.9 \sim 0\end{array}$ & $\begin{array}{l}0.51 \\
3.64 \sim 0\end{array}$ \\
\hline
\end{tabular}


Vol. 4 NORMAL VALUE OF ELEGTROCARDIOGRAM IN THE JAPANESE 16]
No. 2 J $\mathrm{S}$ Deflection (in $\mathrm{mm}$. )

Group (yr.)

\begin{tabular}{|c|c|c|c|c|c|c|c|}
\hline \multicolumn{2}{|c|}{$40 \sim 49$} & \multicolumn{2}{|c|}{$50 \sim 59$} & \multicolumn{2}{|c|}{$60 \sim 69$} & \multicolumn{2}{|c|}{$70 \sim$} \\
\hline $\mathbf{M}$ & $\mathbf{F}$ & $\mathbf{M}$ & $\mathbf{F}$ & $\mathbf{M}$ & $\mathbf{F}$ & $\mathbf{M}$ & $\mathbf{F}$ \\
\hline 153 & 82 & 135 & 102 & 101 & 79 & 48 & 42 \\
\hline 0.79 & 0.40 & 0.76 & 0.66 & 0.56 & 0.54 & 0.68 & 0.56 \\
\hline $5.0 \sim 0$ & $3.1 \sim 0$ & $3.9 \sim 0$ & $3.4 \sim 0$ & $3.4 \sim 0$ & $3.4 \sim 0$ & $3.4 \sim 0$ & $2.4 \sim 0$ \\
\hline 1.14 & 0.48 & 1.14 & 0.64 & 1.37 & 1.07 & 1.18 & 1.04 \\
\hline $9.1 \sim 0$ & $3.7 \sim 0$ & $7.9 \sim 0$ & $4.9 \sim 0$ & $8.4 \sim 0$ & $8.9 \sim 0$ & $5.9 \sim 0.0$ & $4.9 \sim 0$ \\
\hline 1.42 & 0.83 & 1.28 & 1.52 & 1.91 & 2.08 & 2.37 & 2.18 \\
\hline $14.0 \sim 0$ & $5.5 \sim 0$ & $11.9 \sim 0$ & $8.9 \sim 0$ & $9.9 \sim 0$ & $12.9 \sim 0$ & $11.9 \sim 0.0$ & $10.9 \sim 0$ \\
\hline 7.62 & 0.80 & 4.50 & 4.52 & 4.06 & 3.67 & 2.70 & 4.66 \\
\hline $14.0 \sim 0.7$ & $138 \sim 0.1$ & $17.9 \sim 0$ & $15.9 \sim 0$ & $19.9 \sim 0$ & $13.9 \sim 0$ & $11.9 \sim 0$ & $15.9 \sim 0$ \\
\hline 2.44 & 1.26 & 1.83 & 1.50 & 1.76 & 1.21 & 1.12 & 0.88 \\
\hline $8.8 \sim 0$ & $8.2 \sim 0$ & $8.9 \sim 0$ & $5.9 \sim 0$ & $7.9 \sim 0$ & $5.9 \sim 0$ & $5.9 \sim 0.0$ & $3.4 \sim 0$ \\
\hline 1.04 & 0.54 & 1.03 & 0.90 & 1.66 & 1.45 & 1.70 & 1.35 \\
\hline $7.1 \sim 0$ & $3.8 \sim 0$ & $8.9 \sim 0$ & $4.9 \sim 0$ & $22.9 \sim 0$ & $10.9 \sim 0$ & $6.9 \sim 0.0$ & $5.9 \sim 0$ \\
\hline 4.15 & 2.70 & 3.82 & 3.43 & 4.05 & 3.68 & 4.45 & 3.16 \\
\hline $18.6 \sim 0$ & $9.4 \sim 0$ & $13.9 \sim 0$ & $10.9 \sim 0$ & $10.9 \sim 0$ & $20.9 \sim 0$ & $9.9 \sim 0.0$ & $6.9 \sim 0$ \\
\hline 9.42 & 9.62 & 9.58 & 9.07 & 8.83 & 8.46 & 8.87 & 8.47 \\
\hline $24.2 \sim 0$ & $23.3 \sim 1.1$ & $21.9 \sim 0$ & $21.9 \sim 0$ & $19.9 \sim 0$ & $21.9 \sim 0$ & $17.9 \sim 0$ & $17.9 \sim 0$ \\
\hline 13.97 & 12.26 & 14.14 & 13.25 & 12.55 & 13.08 & 13.09 & 12.38 \\
\hline $27.0 \sim 0$ & $30.7 \sim 1.2$ & $31.9 \sim 0$ & $27.9 \sim 0$ & $29.9 \sim 2.0$ & $33.9 \sim 0$ & $29.9 \sim 0.0$ & $27.9 \sim 2.0$ \\
\hline 11.31 & 8.24 & 11.40 & 9.03 & 10.35 & 10.85 & 11.95 & 12.05 \\
\hline $29.5 \sim 0$ & $19.4 \sim 0$ & $27.9 \sim 2.0$ & $27.9 \sim 0$ & $27.9 \sim 0$ & $37.9 \sim 0$ & $27.9 \sim 0$ & $29.9 \sim 0$ \\
\hline 6.43 & 3.91 & 6.82 & 5.85 & 7.01 & 6.36 & 7.66 & 7.38 \\
\hline $20.0 \sim 0$ & $12.7 \sim 0$ & $21.9 \sim 0$ & $21.9 \sim 0$ & $27.9 \sim 0$ & $19.9 \sim 0$ & $27.9 \sim 0$ & $21.9 \sim 0$ \\
\hline 2.46 & 1.46 & 2.53 & 2.06 & 2.75 & 2.80 & 2.26 & 2.50 \\
\hline $11.5 \sim 0$ & $6.3 \sim 0$ & $18.9 \sim 0$ & $10.9 \sim 0$ & $16.9 \sim 0$ & $23.9 \sim 0$ & $7.9 \sim 0$ & $10.9 \sim 0$ \\
\hline 0.64 & 0.39 & 0.91 & 0.72 & 0.99 & 0.94 & 0.80 & 0.84 \\
\hline $6.9 \sim 0$ & $2.6 \sim 0.4$ & $7.9 \sim 0$ & $3.9 \sim 0$ & $9.9 \sim 0$ & $8.9 \sim 0$ & $5.9 \sim 0$ & $4.9 \sim 0$ \\
\hline
\end{tabular}


Table XI. S-T Segment.

\begin{tabular}{|c|c|c|c|c|c|c|c|}
\hline \multirow{2}{*}{\multicolumn{2}{|c|}{$\frac{\text { Age }}{\text { Sex }}$}} & \multicolumn{2}{|c|}{$15 \sim 19$} & \multicolumn{2}{|c|}{$20 \sim 29$} & \multicolumn{2}{|c|}{$30 \sim 39$} \\
\hline & & M & F & $\mathbf{M}$ & $\mathrm{F}$ & $\mathbf{M}$ & $\mathrm{F}$ \\
\hline \multicolumn{2}{|c|}{ Number of Cases } & 60 & 39 & 165 & 109 & 119 & 94 \\
\hline I & $\begin{array}{l}\text { Mn., SD } \\
\text { Max. } \sim \text { Min. }\end{array}$ & $\begin{array}{l}0.03,0.21 \\
0.9 \sim-0.8\end{array}$ & $\begin{array}{c}0.01,0.21 \\
0.7 \sim-0.8\end{array}$ & $\begin{array}{l}0.01,0.25 \\
0.9 \sim-1.0\end{array}$ & $\mid \begin{array}{l}-0.06,0.23 \\
0.05 \sim-0.8\end{array}$ & $\begin{array}{c}0.08,0.17 \\
0.7 \sim-0.8\end{array}$ & $\begin{array}{l}-0.01,0.22 \\
0.9 \sim-0.6\end{array}$ \\
\hline II & $\begin{array}{l}\text { Mn., SD } \\
\text { Max. } \sim \operatorname{Min} .\end{array}$ & $\begin{array}{l}0.02,0.55 \\
2.9 \sim-1.0\end{array}$ & $\begin{array}{r}0.03,0.45 \\
2.4 \sim-0.6 \\
\end{array}$ & $\begin{array}{l}0.13,0.39 \\
1.7 \sim-1.0\end{array}$ & $\begin{array}{l}0.77,0.42 \\
2.1 \sim-0.2\end{array}$ & $\begin{array}{l}0.16,0.38 \\
1.4 \sim-1.0\end{array}$ & $\begin{array}{l}0.00,0.23 \\
0.7 \sim-0.8\end{array}$ \\
\hline III & $\begin{array}{l}\text { Mn., SD } \\
\text { Max, } \sim \text { Min. }\end{array}$ & $\begin{array}{l}0.05,0.28 \\
1.1 \sim-1.2\end{array}$ & $\begin{array}{c}0.01,0.30 \\
1.5 \sim-0.6\end{array}$ & $\begin{array}{l}0.07,0.35 \\
1.9 \sim-0.8\end{array}$ & $\begin{array}{l}0.03,0.40 \\
1.3 \sim-0.8\end{array}$ & $\begin{array}{l}0.15,0.35 \\
1.9 \sim-0.8\end{array}$ & $\begin{array}{l}-0.01,0.30 \\
1.3 \sim-1.0\end{array}$ \\
\hline${ }_{a} V_{\mathrm{I}}$ & $\begin{array}{l}\text { Mn., SD } \\
\text { Max, } \sim \text { Min. }\end{array}$ & $\begin{array}{l}0.06,0.41 \\
1.3 \sim-0.8\end{array}$ & $\begin{array}{l}-0.05,0.20 \\
0.5 \sim-0.6\end{array}$ & $\begin{array}{r}0.00,0.38 \\
2.1 \sim-1.2 \\
\end{array}$ & $\begin{array}{l}-0.05,0.52 \\
1.3 \sim-1.4\end{array}$ & $\begin{array}{l}0.04,0.34 \\
1.1 \sim-1.6 \\
\end{array}$ & $\begin{array}{r}0.01,0.28 \\
1.1 \sim-1.0 \\
\end{array}$ \\
\hline${ }_{\mathrm{a}} \mathrm{V}_{\mathrm{L}}$ & $\begin{array}{l}\text { Mn., SD } \\
\text { Max, } \sim \text { Min. }\end{array}$ & $\begin{array}{l}-0.02,0.23 \\
0.9 \sim-0.8\end{array}$ & $\begin{array}{c}0.03,0.17 \\
0.5 \sim-0.6\end{array}$ & $\begin{array}{l}0.03,0.31 \\
1.3 \sim-1.2\end{array}$ & $\begin{array}{l}0.20,0.35 \\
1.5 \sim-0.6\end{array}$ & $\begin{array}{l}0.04,0.19 \\
0.9 \sim-2.0\end{array}$ & $\begin{array}{l}-0.02,0.27 \\
1.0 \sim-1.8\end{array}$ \\
\hline${ }_{a} V_{F}$ & $\begin{array}{l}\text { Mn., SD } \\
\text { Max. } \sim \text { Min. }\end{array}$ & $\begin{array}{l}0.01,0.35 \\
1.1 \sim-1.0\end{array}$ & $\begin{array}{l}0.03,0.24 \\
1.1 \sim-0.6\end{array}$ & $\begin{array}{l}0.10,0.41 \\
1.8 \sim-1.0\end{array}$ & $\begin{array}{l}0.53,0.35 \\
1.5 \sim-0.4\end{array}$ & $\begin{array}{l}0.16,0.35 \\
2.1 \sim-1.0\end{array}$ & $\begin{array}{l}0.00,0.36 \\
1.5 \sim-1.0\end{array}$ \\
\hline $\mathrm{V}_{4} \mathrm{R}$ & $\begin{array}{l}\text { Mn., SD } \\
\text { Max. - Min. }\end{array}$ & $\begin{array}{c}0.23,0.36 \\
1.1 \sim-0.5 \\
(32) \\
\end{array}$ & $\begin{array}{c}0.00,0.23 \\
1.1 \sim-0.6 \\
(36) \\
\end{array}$ & $\begin{array}{l}0.02,0.24 \\
1.1 \sim-0.6 \\
(114)\end{array}$ & $\begin{array}{c}0.17,0.26 \\
1.1 \sim-0.6 \\
(94)\end{array}$ & $\begin{array}{c}0.11,01.0 \\
0.9 \sim-0.2 \\
(87) \\
\end{array}$ & $\begin{array}{c}-0.02,0.21 \\
1.1 \sim-0.6 \\
(68)\end{array}$ \\
\hline $\mathrm{V}_{1}$ & $\begin{array}{l}\text { Mn., SD } \\
\text { Max. Min. }\end{array}$ & $\begin{array}{c}0.28,0.44 \\
1.3 \sim-0.8\end{array}$ & $\begin{array}{l}0.12,0.35 \\
1.7 \sim-0.8\end{array}$ & $\begin{array}{l}0.44,0.52 \\
2.5 \sim-1.0\end{array}$ & $\begin{array}{l}0.36,0.29 \\
1.1 \sim-0.6\end{array}$ & $\begin{array}{l}0.38,0.44 \\
1.7 \sim-1.6\end{array}$ & $\begin{array}{c}0.09,0.25 \\
0.9 \sim-0.6\end{array}$ \\
\hline $\mathrm{V}_{2}$ & $\begin{array}{l}\text { Mn., SD } \\
\text { Max. } \sim \text { Min. }\end{array}$ & $\begin{array}{l}0.94,0.87 \\
3.3 \sim 0\end{array}$ & $\begin{array}{l}0.26,0.48 \\
1.1 \sim-1.4\end{array}$ & $\begin{array}{l}0.93,0.89 \\
4.1 \sim-1.0\end{array}$ & $\begin{array}{l}0.47,0.31 \\
1.3 \sim-0.4\end{array}$ & $\begin{array}{r}0.70,0.30 \\
4.1 \sim 1.4\end{array}$ & $\begin{array}{l}0.28,0.47 \\
2.7 \sim-0.8\end{array}$ \\
\hline $\mathrm{V}_{3}$ & $\begin{array}{l}\text { Mn., SD } \\
\text { Max. } \sim \text { Min. }\end{array}$ & $\begin{array}{r}1.00,1.57 \\
2.7 \sim-1.5\end{array}$ & $\begin{array}{c}0.47,0.57 \\
2.3 \sim-0.8\end{array}$ & $\begin{array}{r}0.87,0.99 \\
5.5 \sim-0.6\end{array}$ & $\begin{array}{l}0.46,0.29 \\
1.3 \sim-0.4\end{array}$ & $\begin{array}{l}0.67,0.61 \\
2.7 \sim-1.2\end{array}$ & $\begin{array}{l}0.18,0.44 \\
1.9 \sim-1.0\end{array}$ \\
\hline $\mathrm{V}_{4}$ & $\begin{array}{l}\text { Mn., SD } \\
\text { Max. } \sim \text { Min. }\end{array}$ & $\begin{array}{l}0.51,0.72 \\
2.9 \sim-1.0\end{array}$ & $\begin{array}{l}0.10,0.47 \\
2.3 \sim-0.8\end{array}$ & $\begin{array}{l}0.57,0.90 \\
6.4 \sim-1.0\end{array}$ & $\begin{array}{l}0.37,0.23 \\
1.1 \sim-0.4\end{array}$ & $\begin{array}{l}0.59,0.79 \\
5.1 \sim-1.6\end{array}$ & $\begin{array}{l}0.04,0.44 \\
1.7 \sim-0.8\end{array}$ \\
\hline $\mathrm{V}_{\mathrm{s}}$ & $\begin{array}{l}\text { Mn., SD } \\
\text { Max. Min. }\end{array}$ & $\begin{array}{l}0.35,0.50 \\
2.3 \sim-0.4\end{array}$ & $\begin{array}{l}0.03,0.31 \\
1.1 \sim-0.8\end{array}$ & $\begin{array}{l}0.26,0.57 \\
3.0 \sim-1.0\end{array}$ & $\begin{array}{l}0.34,0.22 \\
1.1 \sim-0.2\end{array}$ & $\begin{array}{l}0.25,0.49 \\
2.9 \sim-1.2\end{array}$ & $\begin{array}{l}0.09,0.33 \\
1.3 \sim-0.8\end{array}$ \\
\hline $\mathrm{V}_{6}$ & $\begin{array}{l}\text { Mn., SD } \\
\text { Max. } \sim \text { Min. }\end{array}$ & $\begin{array}{l}0.24,0.50 \\
2.2 \sim 0.4\end{array}$ & $\begin{array}{l}0.00,0.14 \\
0.7 \sim-0.4\end{array}$ & $\begin{array}{r}0.24,0.50 \\
5.1 \sim-0.6\end{array}$ & $\begin{array}{l}0.34,0.25 \\
1.3 \sim-0.2\end{array}$ & $\begin{array}{l}0.21,0.41 \\
2.5 \sim-2.1\end{array}$ & $\begin{array}{l}0.13,0.37 \\
1.5 \sim-1.0\end{array}$ \\
\hline
\end{tabular}


"J" Point (in mm.)

Group (yr.)

\begin{tabular}{|c|c|c|c|c|c|c|c|}
\hline \multicolumn{2}{|c|}{$40 \sim 49$} & \multicolumn{2}{|c|}{$50 \sim 59$} & \multicolumn{2}{|c|}{$60 \sim 69$} & \multicolumn{2}{|l|}{$70 \sim$} \\
\hline M & $F$ & M & $\mathrm{F}$ & & $\mathbf{F}$ & $\mathbf{M}$ & $\mathrm{F}$ \\
\hline & 8 & & & & & 48 & 42 \\
\hline 0.00 & & 22 & $-0.00,0.18$ & $0.06,0.22$ & $0.03,0.10$ & $0.00,0.27$ & \\
\hline-08 & $\sim-$ & $\sim-1.2$ & $5 \sim-0.8$ & $1.1 \sim-1.0$ & $0.7 \sim-0.4$ & $0.9 \sim-1.0$ & $0.3 \sim$ \\
\hline & & 33 & 26 & 41 & 0.06 & $0.00,0.27$ & 0.09 \\
\hline-1.6 & $\sim$ & $7 \sim-$ & $1.1 \sim-$ & .6 & $1.1 \sim-0.6$ & $0.7 \sim-1.0$ & $1.9 \sim$ \\
\hline & & 88 & 3 & 8 & 0.09 & $0.04,0.17$ & 0.03 \\
\hline--1.2 & $1 \sim-$ & $1.0 \sim-$ & $0.9 \sim$ & $1.1 \sim$ & $1.1 \sim-0.6$ & $0.5 \sim-0.4$ & $0.9 \sim-0$ \\
\hline & & & & & 19 & .23 & $-0.08,0$ \\
\hline $1.0 \sim-0.8$ & -1.4 & -1.2 & $0.9 \sim$ & 1.9 & $0.9 \sim-0.8$ & $0.7 \sim-0.6$ & $0.3 \sim-0$ \\
\hline 0.21 & 0.22 & $.04,0.21$ & 13 & 0.17 & $2,0.14$ & 0.13 & 0.02 \\
\hline $1.1 \sim-1.0$ & $0.7 \sim-1.2$ & $0.7 \sim-1.0$ & $0.7 \sim-0.6$ & $1.3 \sim-0.6$ & $0.3 \sim-0.6$ & $0.3 \sim-0.6$ & $0.2 \sim 1.0$ \\
\hline & & & & & & & \\
\hline-1.2 & $\sim-0.6$ & $\sim-1.0$ & $\sim-1$ & $7 \sim-1.6$ & $1.1 \sim-0.6$ & $1.9 \sim-1.0$ & $1.3 \sim-0$ \\
\hline $\begin{array}{c}0.08,0.24 \\
1.1 \sim-1.4 \\
(107)\end{array}$ & $\begin{array}{c}0.02,0.21 \\
.9 \sim-0.8 \\
(74)\end{array}$ & $\begin{array}{c}0.02,0.19 \\
1.7 \sim 0.8 \\
(93)\end{array}$ & $\begin{array}{c}0.07,0.21 \\
1.1 \underset{(78)}{\sim}-1.0\end{array}$ & $\begin{array}{c}0.13,0.14 \\
0.9 \sim 0.4 \\
(74)\end{array}$ & $\begin{array}{c}0.09,0.18 \\
0.9 \sim-2.0 \\
(56)\end{array} \mid$ & $\mid \begin{array}{c}0.16,0.10 \\
0.7 \underset{(23)}{\sim} 0.4\end{array}$ & $0.9 \underset{(17)}{\sim} 0$. \\
\hline & & & 5 & 0.0 & $0.19,0.30$ & $0.46,0.35$ & $0.32,0$. \\
\hline $2.9 \sim-1.4$ & $1.1 \sim-1.2$ & $5 \sim-1.2$ & $1.1 \sim-0.6$ & $2.1 \sim-1.0$ & $1.1 \sim-1.0$ & $.1 \sim-0.5$ & $1.5 \sim-0$ \\
\hline 0.86 & & & & & 0.54 & $.83,0.63$ & 0.62 , \\
\hline 6.11 & $.1 \sim-1.2$ & $8 \sim-2.6$ & $1.9 \sim-1.0$ & -0.5 & $2.7 \sim-1.0$ & $2.5 \sim-$ & $1.5 \sim-0$ \\
\hline $6,1.22$ & $0.11,0.93$ & $0.56,1.25$ & $30,0.62$ & $0,0.37$ & $22,0.40$ & 0.66 & O.T. \\
\hline $9.0 \sim-6.8$ & $2.5 \sim-6.3$ & $2.2 \sim-1.5$ & $2.1 \sim-2.0$ & $2.1 \sim-1.2$ & $1.5 \sim-1.2$ & $2.3 \sim-0.8$ & $2.0 \sim-0$ \\
\hline & & $0.23,0.50$ & & & $0.03,0.43$ & $0.09,0.57$ & $0.00,0$ \\
\hline $3.3 \sim-2.4$ & $2.1 \sim-1.0$ & $1.8 \sim-1.4$ & $2.5 \sim-1.2$ & $1.9 \sim-2.0$ & $1.5 \sim-1.6$ & $1.7 \sim-1.6$ & $1.0 \sim-1$ \\
\hline $0.19,0.41$ & $0.09,0.54$ & $0.05,0.38$ & $0.02,0.35$ & $0.09,0.37$ & $-0.02,0.32$ & $-0.02,0.39$ & (1) \\
\hline $1.9 \sim-2.2$ & $2.5 \sim-1.0$ & $1.5 \sim-1.1$ & $1.5 \sim-1.0$ & $1.5 \sim-1.0$ & $11 \sim-1.0$ & $1.1 \sim-1.2$ & $0.6 \sim-0$ \\
\hline & & $0.10,0.02$ & $0.00,0.20$ & $0.09,0.33$ & $0.09,0.19$ & $0.15,0.30$ & D.0. \\
\hline $1.1 \sim-1.2$ & $2.5 \sim-0.6$ & $1.5 \sim-1.0$ & $1.9 \sim-0.6$ & $1.5 \sim-1.0$ & $1.1 \sim-0.6$ & $0.7 \sim-1.0$ & $1.0 \sim-0$ \\
\hline
\end{tabular}


Table XII. S-T Segment.

\begin{tabular}{|c|c|c|c|c|c|c|c|}
\hline & \multirow{2}{*}{$\frac{\text { Age }}{\text { Sex }}$} & \multicolumn{2}{|c|}{$15 \sim 19$} & \multicolumn{2}{|c|}{$20 \sim 29$} & \multicolumn{2}{|c|}{$30 \sim 39$} \\
\hline & & M & F & $\mathbf{M}$ & $\mathbf{F}$ & $\mathbf{M}$ & F \\
\hline Nun & er of Cases & 61 & 39 & 165 & 109 & 119 & 94 \\
\hline I & $\begin{array}{l}\text { Mn., SD } \\
\text { Max. } \sim \text { Min. }\end{array}$ & $\begin{array}{l}0.56,0.33 \\
1.7-0\end{array}$ & $\begin{array}{c}0.02,0.17 \\
0.7 \sim-0.6\end{array}$ & $\begin{array}{l}0.14,0.22 \\
1.1 \sim-0.6\end{array}$ & $\begin{array}{c}0.06,0.23 \\
0.8 \sim-1.2\end{array}$ & $\begin{array}{c}0.16,0.15 \\
0.9 \sim-0.2\end{array}$ & $\begin{array}{r}0.03,0.13 \\
0.7 \sim-0.2\end{array}$ \\
\hline II & $\begin{array}{l}\text { Mn., SD } \\
\text { Max. } \sim \text { Min. }\end{array}$ & $\begin{array}{l}0.92,0.43 \\
2.0 \sim 0\end{array}$ & $\begin{array}{c}0.03,0.23 \\
0.9 \sim-0.6\end{array}$ & $\begin{array}{l}0.24,0.35 \\
1.5 \sim-0.6\end{array}$ & $\left|\begin{array}{cc}-0.02, & 0.23 \\
1.1 \sim-0.8\end{array}\right|$ & $\begin{array}{c}0.27,0.30 \\
1.1 \sim-0.6\end{array}$ & $\begin{array}{l}0.03,0.18 \\
0.5 \sim-0.6\end{array}$ \\
\hline III & $\begin{array}{l}\text { Mn., SD } \\
\text { Max. } \sim \text { Min. }\end{array}$ & $\begin{array}{r}0.50,0.45 \\
1.7 \sim-1.2\end{array}$ & $\begin{array}{l}-0.05,0.20 \\
0.3 \sim-0.8\end{array}$ & $\begin{array}{c}0.03,0.33 \\
1.1 \sim-1.0\end{array}$ & $\left|\begin{array}{c}-0.03,0.19 \\
0.7 \sim-0.6\end{array}\right|$ & $\begin{array}{c}0.09,0.25 \\
0.9 \sim-0.8\end{array} \mid$ & $\begin{array}{l}0.05,0.17 \\
0.5 \sim-0.8\end{array}$ \\
\hline${ }_{\mathrm{a}} \mathrm{V}_{\mathrm{R}}$ & $\begin{array}{l}\text { Mn., SD } \\
\text { Max. } \sim \text { Min. }\end{array}$ & $\begin{array}{l}-0.62,0.31 \\
-0.1 \sim-1.2\end{array}$ & $\begin{array}{c}-0.06,0.15 \\
0.3 \sim-0.6\end{array}$ & $\mid \begin{array}{l}-0.16,0.35 \\
1.3 \sim-1.2\end{array}$ & $\begin{array}{r}0.04,0.29 \\
1.1 \sim-0.8\end{array}$ & $\left|\begin{array}{c}-0.09,0.25 \\
0.7 \sim-1.2\end{array}\right|$ & $\begin{array}{l}0.02,0.21 \\
0.9 \sim-0.8\end{array}$ \\
\hline${ }_{\mathrm{a}} \mathrm{V}_{\mathrm{L}}$ & $\begin{array}{l}\text { Mn., SD } \\
\text { Max. Min. }\end{array}$ & $\begin{array}{r}0.20,0.36 \\
1.1 \sim-0.9\end{array}$ & $\begin{array}{c}0.04,0.10 \\
0.5 \sim-0.2\end{array}$ & $\begin{array}{c}0.04,0.26 \\
1.1 \sim-0.6\end{array}$ & $\begin{array}{r}0.07,0.58 \\
0.2 \sim-0.6\end{array}$ & $\begin{array}{r}0.12,0.17 \\
0.7 \sim-0.4\end{array}$ & $\begin{array}{c}0.05,0.17 \\
1.1 \sim-0.2\end{array}$ \\
\hline${ }_{\mathrm{a}} \mathrm{V}_{\mathrm{F}}$ & $\begin{array}{l}\text { Mn., SD } \\
\text { Max. } \sim \text { Min. }\end{array}$ & $\begin{array}{c}0.71,0.44 \\
2.3 \sim-0.3\end{array}$ & $\begin{array}{l}-0.03,0.16 \\
0.5 \sim-0.6\end{array}$ & $\begin{array}{r}0.15,0.46 \\
3.7 \sim-3.0\end{array}$ & $\begin{array}{r}0.00,0.19 \\
0.9 \sim-0.6\end{array} \mid$ & $\begin{array}{r}0.05,0.25 \\
0.9 \sim-0.8\end{array} \mid$ & {$\left[\begin{array}{r}0.01,0.19 \\
0.5 \sim-0.8\end{array}\right.$} \\
\hline$V_{4 R}$ & $\begin{array}{l}\text { Mn., SD } \\
\text { Max. } \sim \text { Min. }\end{array}$ & $\begin{array}{l}0.25,0.31 \\
1.1 \underset{(50)}{-} 0.5\end{array}$ & $\begin{array}{c}0.05,0.15 \\
0.7 \sim-0.2 \\
(37)\end{array}$ & $\begin{array}{c}0.17,0.28 \\
1.1 \sim-0.2 \\
(123)\end{array}$ & $\begin{array}{c}0.03,0.16 \\
1.1 \sim-0.2 \\
(95)\end{array}$ & $\begin{array}{c}0.07,0.17 \\
1.6 \sim 0 \\
(84)\end{array}$ & $\begin{array}{c}0.05,0.12 \\
0.5 \sim-0.2 \\
(68)\end{array}$ \\
\hline$V_{1}$ & $\begin{array}{l}\text { Mn., SD } \\
\text { Max. } \sim \text { Min. }\end{array}$ & $\begin{array}{l}0.59,0.36 \\
1.4 \sim-0.3\end{array}$ & $\begin{array}{l}0.37,0.50 \\
1.5 \sim 0\end{array}$ & $\begin{array}{r}0.14,0.58 \\
2.5 \sim-1.0\end{array} \mid$ & $\begin{array}{c}0.38,0.42 \\
2.1 \sim-0.2\end{array} \mid$ & $\begin{array}{l}0.87,0.51 \\
2.9 \sim 0\end{array}$ & $\begin{array}{l}0.37,0.36 \\
1.3 \sim-0.2\end{array}$ \\
\hline $\mathrm{V}_{2}$ & $\begin{array}{l}\text { Mn., SD } \\
\text { Max, } \sim \text { Min. }\end{array}$ & $\begin{array}{l}0.68,0.42 \\
2.0 \sim 0\end{array}$ & $\begin{array}{l}0.93,0.59 \\
2.1 \sim-0.2\end{array}$ & $\begin{array}{r}2.51,1.09 \\
5.5 \sim-0.6\end{array}$ & $\begin{array}{l}0.78,0.60 \\
3.1 \sim 0\end{array}$ & $\mid \begin{array}{l}1.77,0.76 \\
6.7 \sim 0\end{array}$ & $\begin{array}{l}0.89,0.50 \\
2.0 \sim 0\end{array}$ \\
\hline $\mathrm{V}_{3}$ & $\begin{array}{l}\text { Mn:; SD } \\
\text { Max, Min. }\end{array}$ & $\begin{array}{l}0.63,0.36 \\
1.7 \sim 0\end{array}$ & $\begin{array}{l}0.74,0.57 \\
1.7 \sim-0.2\end{array}$ & $\begin{array}{l}1.94,0.77 \\
5.7 \sim 0\end{array}$ & $\begin{array}{c}0.58,0.50 \\
2.1 \sim-0.4\end{array}$ & $\left|\begin{array}{r}1.57,0.77 \\
3.7 \sim-0.4\end{array}\right|$ & $\begin{array}{rr}0.67, & 0.49 \\
2.3 \sim-0.2\end{array}$ \\
\hline$V_{4}$ & $\begin{array}{l}\text { Mn:, SD } \\
\text { Max. } \sim \text { Min. }\end{array}$ & $\begin{array}{l}0.60,0.34 \\
1.7 \sim 0\end{array}$ & $\begin{array}{l}0.41,0.32 \\
1.3 \sim-0.2\end{array}$ & $\begin{array}{l}1.09,0.81 \\
4.5 \sim-0.2\end{array}$ & $\begin{array}{l}0.28,0.4 \\
1.5 \sim-0.4\end{array}$ & $\left|\begin{array}{cc}0.91, & 0.70 \\
3.7 \sim-1.2\end{array}\right|$ & $\begin{array}{l}0.34,0.48 \\
2.9 \sim-0.4\end{array}$ \\
\hline$V_{5}$ & $\begin{array}{l}\text { Mn., SD } \\
\text { Max. Min. }\end{array}$ & $\begin{array}{l}0.50,0.37 \\
1.4 \sim 0\end{array}$ & $\begin{array}{l}0.19,0.26 \\
1.1 \sim-0.2\end{array}$ & $\begin{array}{c}0.56,0.59 \\
3.3 \sim-0.4\end{array}$ & $\begin{array}{l}0.13,0.32 \\
1.5 \sim-1.6\end{array}$ & $\begin{array}{rr}0.45, & 0.45 \\
2.1 & -0.4\end{array} \mid$ & $\begin{array}{l}0.22,0.41 \\
2.5 \sim-0.4\end{array}$ \\
\hline$v_{6}$ & $\begin{array}{l}\text { Mn., SD } \\
\text { Max. } \sim \text { Min. }\end{array}$ & $\begin{array}{l}0.47,0.34 \\
1.7 \sim 0\end{array}$ & $\begin{array}{c}0.04,0.11 \\
0.5 \sim-0.2\end{array}$ & $\begin{array}{r}0.13,0.48 \\
2.9 \sim-0.4\end{array}$ & $\begin{array}{c}0.14,0.30 \\
2.1 \sim-0.4\end{array} \mid$ & $\left|\begin{array}{c}0.25,0.30 \\
2.7 \sim-0.2\end{array}\right|$ & $\begin{array}{l}0.66,0.36 \\
2.3 \sim-0.3\end{array}$ \\
\hline
\end{tabular}


S-T 0.04 Sec. (in $\mathrm{mm}$.)

Group (yr.)

\begin{tabular}{|c|c|c|c|c|c|c|c|}
\hline \multicolumn{2}{|c|}{$40 \sim 49$} & \multicolumn{2}{|c|}{$50 \sim 59$} & \multicolumn{2}{|c|}{$60 \sim 69$} & \multicolumn{2}{|c|}{$70 \sim$} \\
\hline M & $\mathrm{F}$ & $\mathbf{M}$ & $\mathrm{F}$ & M & $\mathrm{F}$ & $\mathbf{M}$ & $\mathrm{F}$ \\
\hline 153 & 82 & 135 & 102 & 101 & 79 & 48 & 42 \\
\hline $0.15,0.15$ & $-0.01,0.15$ & $0.07,0.19$ & $0.05,0.16$ & $0.07,0.10$ & $0.06,0.06$ & $0.03,0.27$ & $0.00,0.10$ \\
\hline $1.1 \sim-0.6$ & $0.5 \sim-0.1$ & $1.0 \sim-0.5$ & $0.5 \sim-0.8$ & $0.7 \sim-0.4$ & $0.5 \sim-0.2$ & $0.7 \sim-1.0$ & $0.4 \sim-0.2$ \\
\hline $0.16,0.32$ & $0.05,0.23$ & $0.08,0.29$ & $0.06,0.25$ & $0.13,0.51$ & $0.09,0.12$ & $0.05,0.12$ & $-0.01,0.19$ \\
\hline $1.5 \sim-1.4$ & $1.1 \sim-0.6$ & $1.0 \sim-1.0$ & $1.1 \sim-0.8$ & $4.3 \sim-1.0$ & $1.1 \sim-0.2$ & $0.7 \sim-1.0$ & $0.5 \sim-0.5$ \\
\hline$-0.01,0.32$ & $0.03,0.16$ & $0.00,0.20$ & $0.06,0.21$ & $0.07,0.32$ & $0.06,0.13$ & $0.03,0.15$ & $0.00,0.12$ \\
\hline $2.5 \sim-1.2$ & $0.5 \sim-0.8$ & $0.6 \sim-0.8$ & $1.1 \sim-0.6$ & $1.1 \sim-2.0$ & $0.7 \sim-0.4$ & $0.3 \sim-0.4$ & $0.5 \sim-0.3$ \\
\hline$-0.05,0.35$ & $0.18,0.16$ & $-0.07,0.23$ & $0.08,0.18$ & $-0.01,0.28$ & $0.02,0.13$ & $0.01,0.17$ & $-0.01,0.13$ \\
\hline $1.9 \sim-1.2$ & $0.5 \sim 0.6$ & $1.1 \sim-0.9$ & $0.9 \sim-0.6$ & $1.9 \sim-0.8$ & $0.3 \sim-0.8$ & $0.5 \sim-0.6$ & $0.3 \sim-0.5$ \\
\hline $0.12,0.10$ & $0.01,0.08$ & $0.05,0.17$ & $0.06,0.06$ & $0.09,0.15$ & $0.04,0.05$ & $0.05,0.14$ & $0.03,0.09$ \\
\hline $1.1 \sim-0.4$ & $0.3 \sim-0.6$ & $1.1 \sim-0.5$ & $0.7 \sim-0.2$ & $1.3 \sim-0.4$ & $0.3 \sim-0.4$ & $0.5 \sim-0.6$ & $0.5 \sim 0.0$ \\
\hline $0.13,0.28$ & $0.04,0.21$ & $0.03,0.23$ & $0.04,0.16$ & $0.07,0.30$ & $0.08,0.12$ & $0.04,0.19$ & $-0.02,0.14$ \\
\hline $1.5 \sim-1.6$ & $0.7 \sim-1.0$ & $1.0 \sim-0.7$ & $0.9 \sim-0.6$ & $1.9 \sim-16$ & $0.7 \sim-0.2$ & $0.5 \sim-0.6$ & $0.4 \sim-0.5$ \\
\hline $\begin{array}{c}0.25,0.19 \\
1.1 \sim-0.6 \\
(108)\end{array}$ & $\begin{array}{c}0.04,0.14 \\
0.9 \sim-0.4 \\
(73)\end{array}$ & $\begin{array}{c}0.12,0.23 \\
1.2 \sim-0.2 \\
(93)\end{array}$ & $\begin{array}{c}0.05,0.21 \\
1.3 \sim-4.2 \\
\quad(78)\end{array}$ & $\begin{array}{c}0.17,0.08 \\
0.9 \sim 0 \\
(74)\end{array}$ & $\begin{array}{c}0.11,0.13 \\
0.7 \sim-0.6 \\
(56)\end{array}$ & $\begin{array}{c}0.39,0.15 \\
0.9 \sim 0.0 \\
(23)\end{array}$ & $\begin{array}{c}0.12,0.09 \\
0.6 \sim 0.0 \\
(17)\end{array}$ \\
\hline $0.79,0.56$ & $0.39,0.39$ & $0.85,0.45$ & $0.37,0.43$ & $0.64,0.33$ & $0.40,0.30$ & $0.30,0.35$ & $0.53,0.48$ \\
\hline $0.9 \sim-0.4$ & $1.7 \sim 0.2$ & $2.5 \sim-0.1$ & $1.5 \sim-1.2$ & $2.1 \sim-0.2$ & $1.3 \sim-0.2$ & $1.5 \sim 0.0$ & $1.9 \sim 0.0$ \\
\hline $1.60,0.92$ & $1.20,0.55$ & $1.61,1.00$ & $0.89,0.58$ & $1.20,0.47$ & $0.90,0.55$ & $1.43,0.67$ & $0.90,0.57$ \\
\hline $4.9 \sim-2.0$ & $2.5 \sim \sim 0.6$ & $5.0 \sim-1.2$ & $2.3 \sim-0.4$ & $2.7 \sim 0$ & $2.5 \sim-0.4$ & $3.3 \sim 0.4$ & $2.4 \sim-0.5$ \\
\hline $1.59,1.01$ & $0.58,0.54$ & $1.50,0.41$ & $0.72,0.65$ & $1.18,0.30$ & $0.66,0.50$ & $1.33,0.75$ & $0.97,0.49$ \\
\hline $5.1 \sim-2.6$ & $2.5 \sim-1.0$ & $3.7 \sim 0.0$ & $2.9 \sim-1.0$ & $2.9 \sim-0.6$ & $2.5 \sim-0.2$ & $3.3 \sim-0.3$ & $2.5 \sim-0.5$ \\
\hline $0.90,0.72$ & $0.24,0.38$ & $0.91,0.77$ & $0.30,0.47$ & $0.72,0.45$ & $0.34,0.67$ & $0.58,0.70$ & $0.35,0.52$ \\
\hline $3.5 \sim-1.6$ & $1.5 \sim-0.6$ & $3.8 \sim-0.5$ & $2.1 \sim-0.8$ & $2.1 \sim-1.0$ & $2.1 \sim-1.0$ & $2.3 \sim-1.0$ & $2.0 \sim-0.3$ \\
\hline $0.45,0.56$ & $0.13,0.27$ & $0.36,0.36$ & $0.16,0.25$ & $0.27,0.36$ & $0.08,0.24$ & $0.15,0.45$ & $0.06,0.27$ \\
\hline $3.3 \sim-1.8$ & $1.1 \sim-0.6$ & $1.5 \sim-0.8$ & $2.1 \sim-0.6$ & $1.3 \sim-0.6$ & $1.1 \sim-0.8$ & $1.3 \sim-1.0$ & $1.0 \sim-0.6$ \\
\hline $0.22,0.28$ & $0.06,0.17$ & $0.15,0.30$ & $0.10,0.10$ & $0.14,0.28$ & $0.07,0.09$ & $-0.02,0.34$ & $0.03,0.16$ \\
\hline $1.5 \sim-1.2$ & $0.7 \sim-0.4$ & $1.3 \sim-0.5$ & $4.1 \sim-0.4$ & $1.1 \sim-1.0$ & $0.5 \sim-0.4$ & $0.7 \sim-3.4$ & $0.5 \sim-0.4$ \\
\hline
\end{tabular}


Table XIII. Voltage of

\begin{tabular}{|c|c|c|c|c|c|c|c|}
\hline \multirow{2}{*}{\multicolumn{2}{|c|}{$\mathrm{Sge}_{\mathrm{Sex}}^{\mathrm{Age}}$}} & \multicolumn{2}{|c|}{$15 \sim 19$} & \multicolumn{2}{|c|}{$20 \sim 29$} & \multicolumn{2}{|c|}{$30 \sim 39$} \\
\hline & & $\mathrm{M}$ & $\mathrm{F}$ & $\mathbf{M}$ & $\mathrm{F}$ & $M$ & $\mathrm{~F}$ \\
\hline \multicolumn{2}{|c|}{ Number of Cases } & 61 & 38 & 165 & 109 & 119 & 94 \\
\hline I & $\begin{array}{l}\text { Mn., SD } \\
M_{a x} \sim M i n .\end{array}$ & $\begin{array}{l}3.21,1.13 \\
5.9 \sim 1.0\end{array}$ & $\begin{array}{l}2.50,0.88 \\
4.9 \sim 0.0\end{array}$ & $\begin{array}{l}2.80,1.11 \\
7.9 \sim 0.0\end{array}$ & $\begin{array}{l}2.42,0.98 \\
5.9 \sim 0.0\end{array}$ & $\begin{array}{l}2.42,0.93 \\
5.9 \sim 0.0\end{array}$ & $\begin{array}{r}2.30,0.79 \\
5.9 \sim-1.0\end{array}$ \\
\hline II & $\begin{array}{l}\text { Mn., SD } \\
\text { Max. } \sim \text { Min. }\end{array}$ & $\begin{array}{l}4.65,1.34 \\
7.9 \sim 1.0\end{array}$ & $\begin{array}{l}2.88,1.04 \\
4.9 \sim 0.0\end{array}$ & $\begin{array}{l}4.26,1.56 \\
9.9 \sim 0.0\end{array}$ & $\begin{array}{l}2.98,1.40 \\
7.9 \sim-3.0\end{array}$ & $\begin{array}{l}3.79,1.47 \\
9.9 \sim 0.0\end{array}$ & $\begin{array}{l}2.91,1.15 \\
6.1 \sim 0.0\end{array}$ \\
\hline III & $\begin{array}{l}\text { Mn., SD } \\
\text { Max. } \sim \text { Min. }\end{array}$ & $\begin{array}{r}1.93,0.99 \\
4.9 \sim-1.0\end{array}$ & $\begin{array}{l}0.42,0.95 \\
3.9 \sim-3.0\end{array}$ & $\begin{array}{r}1.78,1.30 \\
5.9 \sim-2.0\end{array}$ & $\begin{array}{r}0.58,1.10 \\
4.9 \sim-2.0\end{array}$ & $\begin{array}{r}1.65,1.33 \\
5.9 \sim-2.0\end{array}$ & $\begin{array}{l}0.70,0.76 \\
2.9 \sim-2.0\end{array}$ \\
\hline${ }_{\mathrm{a}} V_{R}$ & $\begin{array}{l}\text { Mn., SD } \\
\text { Max. } \sim \mathrm{Min} .\end{array}$ & $\begin{array}{l}-3.68,1.58 \\
-0.1 \sim-70 \\
\end{array}$ & $\begin{array}{c}2.42,0.83 \\
-0.1 \sim-5.0\end{array}$ & $\begin{array}{r}-2.37,1.38 \\
-0.1 \sim \\
-12.0 \\
\end{array}$ & $\begin{array}{l}-0.3,0.95 \\
-0.1 \sim-5.0\end{array}$ & $\begin{array}{l}-2.74,1.46 \\
3.9 \sim-7.6\end{array}$ & $\begin{array}{l}-2.50,0.87 \\
-0.1 \sim-5.0 \\
\end{array}$ \\
\hline${ }_{a} V_{L}$ & $\begin{array}{l}\text { Mn., SD } \\
\text { Max. } \sim \text { Min. }\end{array}$ & $\begin{array}{r}1.05,0.83 \\
3.0 \sim-1.0\end{array}$ & $\begin{array}{r}1.26,0.83 \\
3.9 \sim-1.0\end{array}$ & $\begin{array}{c}0.89,1.03 \\
5.9 \sim-2.0\end{array}$ & $\begin{array}{l}1.34,1.02 \\
6.9 \sim-2.0\end{array}$ & $\begin{array}{l}0.70,1.00 \\
3.9 \sim-2.0\end{array}$ & $\begin{array}{l}1.10,0.72 \\
3.9 \sim-1.0\end{array}$ \\
\hline $\mathrm{a}_{\mathrm{F}}$ & $\begin{array}{l}\mathrm{Mn} ., \mathrm{SD} \\
\mathrm{Max} . \sim \mathrm{Min} .\end{array}$ & $\begin{array}{l}3.18,1.28 \\
5.9 \sim 0.0\end{array}$ & $\begin{array}{c}1.73,0.95 \\
4.9 \sim-1.0\end{array}$ & $\begin{array}{r}2.98,1.56 \\
7.9 \sim-6.0\end{array}$ & $\begin{array}{l}1.88,1.20 \\
6.9 \sim-2.0\end{array}$ & $\begin{array}{r}2.62,1.46 \\
6.9 \sim-4.0\end{array}$ & $\begin{array}{r}1.86,0.93 \\
4.9 \sim-1.0\end{array}$ \\
\hline $\mathrm{V}_{4} \mathrm{R}$ & $\begin{array}{l}\text { Mn., SD } \\
\text { Max. } \sim \text { Min. }\end{array}$ & $\begin{array}{c}-0.26,1.10 \\
1.9 \sim-4.0 \\
(50)\end{array}$ & $\begin{array}{c}-9.55,0.78 \\
1.9 \sim-2.0 \\
(37)\end{array}$ & $\begin{array}{c}0.17,1.25 \\
3.9 \sim-4.0 \\
(117)\end{array}$ & $\begin{array}{c}-0.68,0.95 \\
1.9 \sim-3.0 \\
(96)\end{array}$ & $\begin{array}{c}0.35,1.02 \\
2.9 \sim-3.0 \\
(86)\end{array}$ & $\begin{array}{c}0.67,1.07 \\
11.9 \sim-3.0 \\
(67)\end{array}$ \\
\hline $\mathrm{V}_{1}$ & $\begin{array}{l}\text { Mn., SD } \\
\text { Max. Min. }\end{array}$ & $\begin{array}{r}1.42,2.55 \\
7.9 \sim-5.0\end{array}$ & $\left|\begin{array}{c}-0.15,1.3 \varepsilon \\
2.9 \sim-4.0\end{array}\right|$ & $\begin{array}{rr}1.99, & 1.81 \\
6.9 \sim-3.0\end{array}$ & $\left|\begin{array}{l}-0.45,1.65 \\
4.9 \sim-4.0\end{array}\right|$ & $\begin{array}{l}1.77,2.02 \\
8.9 \sim-2.0\end{array}$ & $\begin{array}{l}-0.19,1.34 \\
2.9 \sim-3.0\end{array}$ \\
\hline $\mathrm{V}_{2}$ & $\begin{array}{l}\mathrm{Mn}, \mathrm{SD} \\
\mathrm{M}_{\mathrm{ax}}, \sim \mathrm{Min}_{\text {in }}\end{array}$ & $\begin{array}{c}8.38,3.67 \\
19.9 \sim-1.0\end{array}$ & $\begin{array}{c}3.20,2.90 \\
10.9 \sim-3.0\end{array} \mid$ & $\begin{array}{l}8.14,3.65 \\
23.9 \sim 0.0\end{array}$ & $\begin{array}{c}2.96,2.16 \\
8.9 \sim-4.0\end{array}$ & $\begin{array}{r}6.90,3.57 \\
18.9 \sim-2.0\end{array}$ & $\begin{array}{l}3.17,2.03 \\
8.9 \sim-2.0\end{array}$ \\
\hline $\mathrm{V}_{3}$ & $\begin{array}{l}\mathrm{Mn} ., \mathrm{SD} \\
\mathrm{Max} . \sim \mathrm{Min} .\end{array}$ & $\begin{array}{l}9.92,3.52 \\
20.9 \sim 0.0\end{array}$ & $\begin{array}{l}4.50,2.24 \\
10.9 \sim 0.0\end{array}$ & $\begin{array}{l}8.86,3.63 \\
22.9 \sim 2.0\end{array}$ & $\begin{array}{l}4.05,2.16 \\
9.9 \sim-0.0\end{array}$ & $\begin{array}{l}7.58,3.37 \\
20.9 \sim 1.0\end{array}$ & $\begin{array}{l}4.21,2.17 \\
10.9 \sim 0.0\end{array}$ \\
\hline $\mathrm{V}_{4}$ & $\begin{array}{l}\mathrm{Mn}, \mathrm{SD} \\
\mathrm{Max} . \sim \mathrm{Min} .\end{array}$ & $\begin{array}{l}9.38,3.75 \\
16.9 \sim 1.0\end{array}$ & $\begin{array}{l}5.15,2.08 \\
10.9 \sim 0.0\end{array}$ & $\begin{array}{l}8.72,3.93 \\
24.9 \sim 1.0\end{array}$ & $\begin{array}{l}4.80,1.34 \\
10.9 \sim 0.0\end{array}$ & $\begin{array}{l}7.58,3.89 \\
40.9 \sim 1.0\end{array}$ & $\begin{array}{l}4.87,2.46 \\
13.0 \sim 0.0\end{array}$ \\
\hline$V_{5}$ & $\begin{array}{l}\text { Mn., SD } \\
\text { Max. } \sim \text { Min. }\end{array}$ & $\begin{array}{l}8.53,3.58 \\
15.9 \sim 0.0\end{array}$ & $\begin{array}{l}4.45,1.70 \\
8.9 \sim 0.0\end{array}$ & $\begin{array}{l}5.30,2.82 \\
16.9 \sim 1.0\end{array}$ & $\begin{array}{r}4.25,1.65 \\
10.9 \sim-2.0\end{array}$ & $\begin{array}{l}5.44,2.86 \\
14.9 \sim 0.0\end{array}$ & $\begin{array}{l}4.45,2.01 \\
11.0 \sim 0.0\end{array}$ \\
\hline$V_{6}$ & $\begin{array}{l}\text { Mn., SD } \\
\text { Max. } \sim \text { Min. }\end{array}$ & $\begin{array}{l}4.96,2.21 \\
11.9 \sim 0.0\end{array}$ & $\begin{array}{l}3.69,1.37 \\
6.9 \sim 0.0\end{array}$ & $\begin{array}{l}4.48,2.15 \\
14.9 \sim 0.0\end{array}$ & $\begin{array}{l}3.43,2.41 \\
8.9 \sim 0.0\end{array}$ & $\begin{array}{l}3.80,1.85 \\
8.9 \sim 0.0\end{array}$ & $\begin{array}{l}3.55,1.45 \\
7.9 \sim 1.0\end{array}$ \\
\hline
\end{tabular}


' $\Gamma$ Deflection (in $\mathrm{mm}$.)

Group (yr.)

\begin{tabular}{|c|c|c|c|c|c|c|c|}
\hline \multicolumn{2}{|c|}{$40 \sim 49$} & \multicolumn{2}{|c|}{$50 \sim 59$} & \multicolumn{2}{|c|}{$60 \sim 6$} & \multicolumn{2}{|c|}{$70 \sim$} \\
\hline M & $\mathrm{F}$ & M & $\mathrm{F}$ & M & $\mathrm{F}$ & $\mathbf{M}$ & F \\
\hline & 82 & & & & & 48 & 42 \\
\hline & & 3 & 1 & & & $1.54,1.74$ & 1.83, \\
\hline 6.9 & & $5.9 \sim 1$ & & 0 & $4.9 \sim 0.0$ & $2.9 \sim-1.0$ & $4.9-$ \\
\hline & & & & & & $.56,1.17$ & \\
\hline 7.9 & $9 \sim$ & $8.9 \sim-1.0$ & $5.9 \sim$ & $7.9 \sim-2.0$ & 7.9 - & 0 & \\
\hline & & & & & $0.54,1.07$ & 14 & $0.62,0.78$ \\
\hline $6.9 \sim-3.0$ & $4.9 \sim-2.0$ & $5.9 \sim-2.0$ & $2.9 \sim-2.0$ & $5.9 \sim-3.0$ & $3.9 \sim-2.0$ & $4.9 \sim$ & $2.9 \sim-2.0$ \\
\hline $.00,2.28$ & $3-1.55,1.53$ & $-2.31,1.42$ & $-1.88,1.1 \mathrm{C}$ & 43 & $-1.58,1.16$ & $-0.16,1.17$ & \\
\hline 4.9 & 3.9 & $4.9 \sim$ & .0 & 0. & $2.9 \sim-4.0$ & $1.9 \sim-5.0$ & $2.9 \sim$ \\
\hline & & & $0.96,0.72$ & $0.52,1.15$ & $0.27,0.84$ & $.52,0.71$ & $0.90,0.85$ \\
\hline $7.9 \sim-2$. & .0 & $3.9 \sim-2.0$ & $2.9 \sim-1.0$ & $3.9 \sim-3.0$ & $3.9 \sim$ & $1.9 \sim$ & $3.9 \sim$ \\
\hline & $72,0.90$ & 26 & & & & & \\
\hline & 2 & & $4.9 \sim-1.0$ & $6.9 \sim-2.0$ & $3.9 \sim-1.0$ & $4.9 \sim-1.0$ & $3.9 \sim$ \\
\hline $\begin{array}{l}0.01,1.26 \\
.9 \sim-5.0 \\
(108) \\
\end{array}$ & $\begin{array}{l}-0.4 \\
2.9 \\
(7\end{array}$ & $\begin{array}{c}-0.02,0.96 \\
3.9 \sim-3.0 \\
(94)\end{array}$ & $\begin{array}{c}-0.42,0.81 \\
1.9 \sim 2.0 \\
(78)\end{array}$ & $\begin{array}{c}0.26,0.99 \\
9 \sim-3.0 \\
(74) \\
\end{array}$ & $\begin{array}{c}-0.55,0.84 \\
2.9 \sim-3.0 \\
-(56) \\
\end{array}$ & $\begin{array}{c}00,1.16 \\
9 \sim-2.0 \\
(23)\end{array}$ & $\begin{array}{c}-0.32,0.57 \\
0.9 \sim 2.0 \\
(17)\end{array}$ \\
\hline & & $1.35,2.10$ & -0.13 & 1.20 , & .08 , & .67 & $0.29,1.86$ \\
\hline 10. & .0 & $7.9 \sim-5.0$ & & $5.9 \sim-3.0$ & $4.9 \sim-3.0$ & $5.9 \sim-3.0$ & $6.9 \sim-$ \\
\hline & & & & & $3.08,2.19$ & .80 & $3.24,2$. \\
\hline $13.9 \sim-1.0$ & & -3.0 & $9.9 \sim-3.0$ & $11.9 \sim-5.0$ & $10.9 \sim-2.0$ & $11.9 \sim 0.0$ & $9.9 \sim-3$ \\
\hline & & & & $7.27,2.98$ & $4.36,2.37$ & $7.08,2.98$ & $4.62,2.4$ \\
\hline & $9.9 \sim$ & $18.9 \sim 2.0$ & $12.9 \sim-3.0$ & $15.9 \sim-2.0$ & $11.9 \sim-1.0$ & $14.9 \sim 2.0$ & $8.9 \sim-1$ \\
\hline & & $3,2.93$ & & $7.07,3.27$ & $4.22,1.99$ & $6.19,2.63$ & 4.33 \\
\hline 26.9 & $11.9 \sim-1.0$ & $15.9 \sim-2.0$ & $13.9 \sim-2.0$ & $17.4 \sim-5.0$ & $12.9 \sim 0.0$ & $3.9 \sim-4.0$ & $11.9 \sim$ \\
\hline & & & & $5.48,2.51$ & $3.32,1.77$ & $4.42,2.49$ & $0.1,18$ \\
\hline & & $14.9 \sim-2.0$ & $9.9 \sim 0.0$ & $12.9 \sim 0.0$ & $9.9 \sim 0.0$ & $10.9 \sim-2.0$ & $8.9 \sim 0.0$ \\
\hline & & & & & & & \\
\hline & $7.9 \sim 0.0$ & $8.9 \sim-1.0$ & $7.9 \sim 0.0$ & $13.9 \sim 0.0$ & $4.9 \sim 0.0$ & $6.9 \sim-1.0$ & \\
\hline
\end{tabular}


Table XIV. Voltage of U

\begin{tabular}{|c|c|c|c|c|c|c|c|}
\hline & \multirow{3}{*}{$\underbrace{\text { Age }}_{\text {Sex }}$} & & & & & \multicolumn{2}{|r|}{ Age } \\
\hline & & \multicolumn{2}{|c|}{$15 \sim 19$} & \multicolumn{2}{|c|}{$20 \sim 29$} & \multicolumn{2}{|c|}{$30 \sim 39$} \\
\hline & & $\mathbf{M}$ & $\mathrm{F}$ & $\mathbf{M}$ & $F$ & $\mathrm{M}$ & $\mathbf{F}$ \\
\hline \multicolumn{2}{|c|}{ Number of Cases } & 61 & 39 & 165 & 109 & 119 & 94 \\
\hline $\mathrm{I}$ & $\begin{array}{l}\text { Mn. } \\
\text { Max. } \sim \operatorname{Min} .\end{array}$ & $\begin{array}{c}0.08 \\
0.3 \sim 0 \\
(36) \\
\end{array}$ & $\begin{array}{c}0.04 \\
0.2 \sim 0 \\
(25)\end{array}$ & $\begin{array}{c}0.06 \\
1.5 \sim-0.1 \\
(110)\end{array}$ & $\begin{array}{c}1.07 \\
2.0 \sim 0 \\
(77)\end{array}$ & $\begin{array}{c}0.04 \\
0.8 \sim-0.2 \\
(94)\end{array}$ & $\begin{array}{c}0.03 \\
0.4 \sim 0 \\
(72)\end{array}$ \\
\hline II & $\begin{array}{l}\text { Mn. } \\
\text { Max. } \sim \operatorname{Min} .\end{array}$ & $\begin{array}{c}0.16 \\
1.0 \sim 0 \\
(47)\end{array}$ & $\begin{array}{c}0.10 \\
0.4 \sim 0 \\
(25) \\
\end{array}$ & $\begin{array}{l}0.19 \\
1.5 \sim 0 \\
(127)\end{array}$ & $\begin{array}{c}0.14 \\
1.0 \sim 0 \\
(82)\end{array}$ & $\begin{array}{c}0.13 \\
0.9 \sim 0 \\
(103)\end{array}$ & $\begin{array}{c}0.09 \\
0.6 \sim 0 \\
(78)\end{array}$ \\
\hline III & $\begin{array}{l}\text { Mn. } \\
\text { Max. } \sim \operatorname{Min} .\end{array}$ & $\begin{array}{c}0.09 \\
0.8 \sim 0 \\
(31)\end{array}$ & $\begin{array}{c}0.04 \\
0.2 \sim 0.1 \\
(24)\end{array}$ & $\begin{array}{c}0.07 \\
0.6-0 \\
(111)\end{array}$ & $\begin{array}{c}0.07 \\
0.9 \sim 0 \\
(73)\end{array}$ & $\begin{array}{c}0.06 \\
0.4 \sim 0 \\
(98)\end{array}$ & $\begin{array}{c}0.05 \\
0.5 \sim 0 \\
(73)\end{array}$ \\
\hline $\mathrm{V}_{\mathrm{R}}$ & $\begin{array}{l}\text { Mn. } \\
\text { Max. } \sim \operatorname{Min} .\end{array}$ & $\begin{array}{c}-0.07 \\
0 \sim-0.5 \\
(31)\end{array}$ & $\begin{array}{c}-0.05 \\
0.1 \sim 0.3 \sim \\
(22)\end{array}$ & $\begin{array}{c}0.02 \\
0.4 \sim-1.0 \\
(113)\end{array}$ & $\begin{array}{c}0.02 \\
0.5 \sim-0.5 \\
(74)\end{array}$ & $\begin{array}{c}-0.02 \\
0.2 \sim-0.5 \\
(99)\end{array}$ & $\begin{array}{c}-0.01 \\
0.2 \sim-0.3 \\
(66)\end{array}$ \\
\hline${ }_{\mathrm{N}} \mathrm{V}_{\mathrm{L}}$ & $\begin{array}{l}\text { Mn. } \\
\text { Max. Min. }\end{array}$ & $\begin{array}{c}0.04 \\
0.3 \sim 0 \\
(33)\end{array}$ & $\begin{array}{c}0.02 \\
0.1 \sim 0 \\
(22)\end{array}$ & $\begin{array}{c}0.02 \\
0.4 \sim 0 \\
(107)\end{array}$ & $\begin{array}{c}0.01 \\
0.3 \sim-0.2 \\
(67)\end{array}$ & $\begin{array}{c}0.00 \\
0.1 \sim-0.1 \\
(89)\end{array}$ & $\begin{array}{c}0.01 \\
0.3 \sim 0 \\
(61)\end{array}$ \\
\hline${ }_{i l} V_{F}$ & $\begin{array}{l}\mathrm{Mn} . \\
\mathrm{Max} . \sim \mathrm{Min} .\end{array}$ & $\begin{array}{c}0.14 \\
0.8 \sim 0 \\
(31)\end{array}$ & $\begin{array}{c}0.05 \\
0.4 \sim 0 \\
(26)\end{array}$ & $\begin{array}{c}0.12 \\
1.0 \sim 0 \\
(116)\end{array}$ & $\begin{array}{l}0.11 \\
1.2 \sim 0 \\
(76)\end{array}$ & $\begin{array}{c}0.10 \\
0.8 \sim 0 \\
(104)\end{array}$ & $\begin{array}{l}0.11 \\
4.0 \sim 0 \\
.(70) \\
\end{array}$ \\
\hline$V_{4} \pi$ & $\begin{array}{l}\text { Mn. } \\
\text { Max. Min. }\end{array}$ & $\begin{array}{c}0.35 \\
3.0 \sim 0 \\
(20)\end{array}$ & $\begin{array}{c}0.01 \\
0.2 \sim-0.02 \\
(21)\end{array}$ & $\begin{array}{c}0.03 \\
0.5 \sim 0 \\
\quad(65)\end{array}$ & $\begin{array}{c}0.00 \\
0.2 \sim 0 \\
(56)\end{array}$ & $\begin{array}{c}0.08 \\
0.6 \sim 0 \\
(67)\end{array}$ & $\begin{array}{c}0.01 \\
0.4 \sim 0 \\
(44)\end{array}$ \\
\hline $\mathrm{V}_{1}$ & $\begin{array}{l}\text { Mn. } \\
\text { Max. Min. }\end{array}$ & $\begin{array}{c}0.40 \\
1.4 \sim 0 \\
(43)\end{array}$ & $\begin{array}{c}0.10 \\
0.8 \sim-0.2 \\
(25)\end{array}$ & $\begin{array}{c}0.35 \\
2.0 \sim-0.5 \\
(134)\end{array}$ & $\begin{array}{c}0.21 \\
2.0 \sim-0.2 \\
(76)\end{array}$ & $\begin{array}{c}0.28 \\
1.0 \sim 0 \\
(103)\end{array}$ & $\begin{array}{c}0.10 \\
1.0 \sim-0.3 \\
(73)\end{array}$ \\
\hline $\mathrm{V}_{2}$ & $\begin{array}{l}\text { Mn. } \\
\text { Max. Min. }\end{array}$ & $\begin{array}{c}1.07 \\
2.5 \sim 0.2 \\
(56)\end{array}$ & $\begin{array}{c}0.41 \\
1.5 \sim 0.2 \\
(35)\end{array}$ & $\begin{array}{c}0.96 \\
2.8 \sim 0 \\
(160)\end{array}$ & $\begin{array}{c}0.69 \\
2.2 \sim 0 \\
(97)\end{array}$ & $\begin{array}{c}0.66 \\
2.1 \sim 0 \\
(1 / 8)\end{array}$ & $\begin{array}{c}0.50 \\
1.7 \sim 0 \\
(86)\end{array}$ \\
\hline $\mathrm{V}_{3}$ & $\begin{array}{l}\text { Mn. } \\
\text { Max. Min. }\end{array}$ & $\begin{array}{c}0.94 \\
2.5 \sim 0 \\
(56)\end{array}$ & $\begin{array}{c}0.48 \\
1.5 \sim 0 \\
(33)\end{array}$ & $\begin{array}{c}0.9 t \\
2.8 \sim 0 \\
(156) \\
\end{array}$ & $\begin{array}{c}0.59 \\
2.9 \sim 0 \\
(96)\end{array}$ & $\begin{array}{c}0.64 \\
3.5 \sim-2.2 \\
(114) \\
\end{array}$ & $\begin{array}{c}0.53 \\
1.6 \sim 0 \\
(85) \\
\end{array}$ \\
\hline $\mathrm{V}_{4}$ & $\begin{array}{l}\text { Mn. } \\
\text { Max. } \sim \text { Min. }\end{array}$ & $\begin{array}{l}0.73 \\
2.1 \sim 0 \\
(51)\end{array}$ & $\begin{array}{c}0.29 \\
1.0 \sim 0 \\
(33)\end{array}$ & $\begin{array}{c}0.65 \\
6.8 \sim 0 \\
(144) \\
\end{array}$ & $\begin{array}{l}0.36 \\
1.9 \sim 0 \\
(93) \\
\end{array}$ & $\begin{array}{c}0.49 \\
2.3 \sim-0.3 \\
(110)\end{array}$ & $\begin{array}{c}0.25 \\
1.2-0 \\
(81)\end{array}$ \\
\hline $\mathrm{V}_{\mathrm{s}}$ & $\begin{array}{l}\text { Mn. } \\
\text { Max. } \sim \operatorname{Min} .\end{array}$ & $\begin{array}{c}0.39 \\
2.0 \sim 0 \\
(44)\end{array}$ & $\begin{array}{c}0.11 \\
0.7 \sim 0 \\
(30)\end{array}$ & $\begin{array}{c}0.31 \\
2.0 \sim 0 \\
(127)\end{array}$ & $\begin{array}{l}0.25 \\
1.5 \sim 0 \\
(88)\end{array}$ & $\begin{array}{c}0.30 \\
7.6 \sim-0.1 \\
(106)\end{array}$ & $\begin{array}{c}0.15 \\
2.0 \sim 0 \\
(79)\end{array}$ \\
\hline$V_{6}$ & $\begin{array}{l}\text { Mn. } \\
\text { Max. Min. }\end{array}$ & $\begin{array}{c}0.22 \\
2.7 \sim 0 \\
(39)\end{array}$ & $\begin{array}{c}0.06 \\
0.3 \sim 0 \\
(27)\end{array}$ & $\begin{array}{c}0.10 \\
0.8 \sim 0 \\
(114)\end{array}$ & $\begin{array}{l}0.10 \\
1.9 \sim 0 \\
(81)\end{array}$ & $\begin{array}{c}0.11 \\
2.0 \sim 0 \\
(110)\end{array}$ & $\begin{array}{c}0.04 \\
0.4 \sim 0 \\
(72)\end{array}$ \\
\hline
\end{tabular}

( ): Number of cases in which $\mathrm{U}$ deffections were measurable. 
Deflection (in mm.)

Group (yr.)

\begin{tabular}{|c|c|c|c|c|c|c|c|}
\hline \multicolumn{2}{|c|}{$40 \sim 49$} & \multicolumn{2}{|c|}{$50 \sim 59$} & \multicolumn{2}{|c|}{$60 \sim 69$} & \multicolumn{2}{|c|}{$70 \sim$} \\
\hline $\mathbf{M}$ & F & $\mathbf{M}$ & $\mathrm{F}$ & $M$ & F & $\mathbf{M}$ & $\mathbf{F}$ \\
\hline 153 & 82 & 135 & 102 & 10 & 79 & 48 & 42 \\
\hline & $\begin{array}{l}0 \\
0 . \\
\end{array}$ & $\begin{array}{c}0.02 \\
0.2 \sim 0 \\
(89)\end{array}$ & $\begin{array}{c}0.03 \\
0.3 \sim 0 \\
(75)\end{array}$ & $\begin{array}{c}0.03 \\
0.4 \sim 0 \\
(65)\end{array}$ & & $\begin{array}{c}0.03 \\
0.3 \sim 0 \\
(37)\end{array}$ & $\begin{array}{c}0.02 \\
0.3 \sim 0.3 \\
(37)\end{array}$ \\
\hline $\begin{array}{c}0.18 \\
6.2 \sim-0.3 \\
(128)\end{array}$ & $\begin{array}{c}0.1 \\
0.5 \\
(6 . \\
\end{array}$ & $\begin{array}{c}0.09 \\
0.6 \sim 0 \\
(98)\end{array}$ & $\begin{array}{c}0.12 \\
1.0 \sim 0 \\
(87)\end{array}$ & & & $\begin{array}{c}0.10 \\
1.0 \sim 0\end{array}$ & $\begin{array}{c}0.18 \\
0.5 \sim-0.3 \\
(37)\end{array}$ \\
\hline $\begin{array}{c}0.05 \\
0.7 \sim-1.1 \\
(122)\end{array}$ & $\begin{array}{r}0.0 \\
0.5 \\
(66 \\
\end{array}$ & $\begin{array}{c}0.04 \\
0.3 \sim 0 \\
(98)\end{array}$ & $\begin{array}{c}0.05 \\
1.0 \sim-0.2 \\
(74)\end{array}$ & $\begin{array}{c}0.09 \\
0.7 \underset{(70)}{\sim}-0.3\end{array}$ & & $\begin{array}{c}0.04 \\
0.3 \sim-0.7 \\
(39)\end{array}$ & $\begin{array}{c}0.05 \\
0.4 \sim 0 \\
(35)\end{array}$ \\
\hline $\begin{array}{c}-0.01 \\
0.4 \sim-0.3 \\
(122)\end{array}$ & $\begin{array}{c}-0.05 \\
0.2 \sim-0.8 \\
(66)\end{array}$ & $\begin{array}{c}-0.03 \\
0.2 \sim-0.6 \\
(91)\end{array}$ & $\begin{array}{c}-0.03 \\
0.0 \sim-0.3 \\
(74)\end{array}$ & $\begin{array}{c}-0.04 \\
0 \sim-0.4 \\
(67)\end{array}$ & $\begin{array}{c}-0.03 \\
0 \sim-0.4 \\
(60)\end{array}$ & $\begin{array}{c}-0.03 \\
0.2 \sim-0.5 \\
(37)\end{array}$ & $\begin{array}{c}0.00 \\
0.3 \sim-0.3 \\
(37)\end{array}$ \\
\hline $\begin{array}{c}0.00 \\
0.3 \sim-0.1 \\
(112)\end{array}$ & $\begin{array}{c}0.02 \\
0.2 \sim 0 \\
(64)\end{array}$ & $\begin{array}{c}0.01 \\
0.5 \sim-0.2 \\
(83)\end{array}$ & $\begin{array}{c}0.01 \\
0.3 \sim 0 \\
(69)\end{array}$ & $\begin{array}{c}0.00 \\
0.3 \sim 0.1 \\
(64)\end{array}$ & $\begin{array}{c}0.00 \\
0.2 \sim-0.3 \\
(61)\end{array}$ & $\begin{array}{c}0.02 \\
0.3 \sim 0 \\
(36)\end{array}$ & $\begin{array}{c}0.01 \\
0.3 \sim 0 \\
(37)\end{array}$ \\
\hline & $\begin{array}{c}0.04 \\
0.4 \sim 0 \\
(66) \\
\end{array}$ & $\begin{array}{c}0.07 \\
0.8 \sim 0 \\
(93)\end{array}$ & & $\begin{array}{c}0.11 \\
1.1 \sim 0 \\
(74)\end{array}$ & $\begin{array}{c}0.08 \\
0.4 \sim 0 \\
(61)\end{array}$ & $\begin{array}{c}0.01 \\
0.2 \sim-0.7 \\
(4.0)\end{array}$ & $\begin{array}{c}0.09 \\
0.3 \sim 0 \\
(37) \\
\end{array}$ \\
\hline $\begin{array}{c}0.02 \\
0.6 \sim 0 \\
(84)\end{array}$ & $\begin{array}{c}0.00 \\
0.2 \sim 0 \\
(55)\end{array}$ & $\begin{array}{c}0.03 \\
0.4 \sim \underset{(65)}{\sim} 0.1\end{array}$ & $\begin{array}{c}0.01 \\
0.4 \sim-0.2 \\
(58)\end{array}$ & $\begin{array}{c}-0.02 \\
0.3 \sim-1.2 \\
(46)\end{array}$ & $\begin{array}{c}0.00 \\
0.3 \sim-0.2 \\
(45)\end{array}$ & $\begin{array}{c}0.01 \\
0.2 \sim-1.2 \\
(15)\end{array}$ & 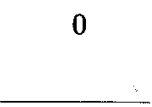 \\
\hline $\begin{array}{c}0.16 \\
1.5 \sim-0.2 \\
(125)\end{array}$ & $\begin{array}{c}0.07 \\
0.9 \sim 0 \\
(81)\end{array}$ & $\begin{array}{c}0.18 \\
1.3 \sim-0.2 \\
(107)\end{array}$ & $\begin{array}{c}0.06 \\
0.5 \sim 0 \\
(75) \\
\end{array}$ & $\begin{array}{c}0.07 \\
1.1 \sim-0.2 \\
(73)\end{array}$ & $\begin{array}{c}0.11 \\
1.0 \sim 0 \\
(59)\end{array}$ & $\begin{array}{c}0.20 \\
1.0 \sim-0.2 \\
(38) \\
\end{array}$ & $\begin{array}{c}0.10 \\
0.7 \underset{-0.1}{(36)} \\
\end{array}$ \\
\hline $\begin{array}{c}0.72 \\
10.0 \sim 0 \\
(144)\end{array}$ & $\begin{array}{c}0.47 \\
1.5 \sim 0 \\
(79)\end{array}$ & $\begin{array}{c}0.53 \\
1.8 \sim 0 \\
(124)\end{array}$ & & $\begin{array}{c}0.42 \\
1.4 \sim 0 \\
(93)\end{array}$ & & $\begin{array}{c}0.48 \\
1.7 \sim 0 \\
(47)\end{array}$ & $\begin{array}{c}0.41 \\
1.1 \sim 0 \\
(38)\end{array}$ \\
\hline $\begin{array}{l}0.80 \\
6.0 \sim 0 \\
(149)\end{array}$ & $\begin{array}{c}0.44 \\
1.7 \sim 0 \\
(78)\end{array}$ & $\begin{array}{c}0.56 \\
1.9 \sim 0 \\
(128)\end{array}$ & $\begin{array}{c}0.43 \\
2.0 \sim 0.2 \\
(96)\end{array}$ & $\begin{array}{c}0.51 \\
1.7 \sim 0 \\
(96)\end{array}$ & $\begin{array}{c}0.48 \\
1.5 \sim-0.6 \\
(72) \\
\end{array}$ & & $\begin{array}{c}0.49 \\
1.6 \sim 0 \\
(39)\end{array}$ \\
\hline $\begin{array}{c}0.49 \\
2.5 \sim-0.2 \\
(140)\end{array}$ & $\begin{array}{c}0.32 \\
1.0 \sim 0 \\
(74)\end{array}$ & $\begin{array}{c}0.37 \\
2.0 \sim 0 \\
(122)\end{array}$ & $\begin{array}{c}0.40 \\
2.0 \sim 0 \\
(96)\end{array}$ & $\begin{array}{c}0.35 \\
1.6 \sim 0 \\
(95)\end{array}$ & $\begin{array}{c}0.30 \\
1.3 \sim 0 \\
(72)\end{array}$ & $\begin{array}{c}0.40 \\
2.0 \sim 0 \\
(41)\end{array}$ & $\begin{array}{c}0.37 \\
1.3 \sim-0.2 \\
(39)\end{array}$ \\
\hline $\begin{array}{c}0.24 \\
1.3 \sim 0 \\
(131)\end{array}$ & $\begin{array}{c}0.16 \\
0.9 \sim 0 \\
(71)\end{array}$ & $\begin{array}{l}0.19 \\
1.0 \sim 0 \\
(111)\end{array}$ & $\begin{array}{c}0.25 \\
2.0 \sim 0 \\
(94)\end{array}$ & $\begin{array}{c}0.20 \\
1.2 \sim 0 \\
(86)\end{array}$ & $\begin{array}{c}0.19 \\
1.0 \sim-0.5 \\
(69)\end{array}$ & $\begin{array}{c}0.19 \\
0.8 \sim-0.8 \\
(40)\end{array}$ & $\begin{array}{c}0.18 \\
1.3 \sim 0.7 \\
(39)\end{array}$ \\
\hline $\begin{array}{l}0.12 \\
1.4 \sim 0 \\
(123)\end{array}$ & $\begin{array}{c}0.05 \\
0.4 \sim 0 \\
(65)\end{array}$ & $\begin{array}{c}0.18 \\
0.8 \sim 0 \\
(95)\end{array}$ & $\begin{array}{c}0.09 \\
0.6 \sim-0.5 \\
(92)\end{array}$ & $\begin{array}{c}0.08 \\
1.0 \sim 0 \\
(73)\end{array}$ & $\begin{array}{c}0.07 \\
0.6 \sim-1.0 \\
(69)\end{array}$ & $\begin{array}{c}0.09 \\
0.7 \sim 0 \\
(37)\end{array}$ & $\begin{array}{c}0.08 \\
0.7 \sim-0 \\
(38)\end{array}$ \\
\hline
\end{tabular}


Table XV. Ventricular Activation Time (in sec.)

\begin{tabular}{|c|c|c|c|c|c|c|c|c|c|}
\hline \multicolumn{10}{|c|}{ Age Group (yr.) } \\
\hline \multirow[t]{2}{*}{ 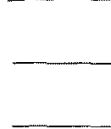 } & \multirow{2}{*}{$\frac{\text { Age }}{\text { Sex }}$} & \multicolumn{2}{|c|}{$15 \sim$} & \multicolumn{2}{|c|}{$20 \sim$} & \multicolumn{2}{|c|}{$30 \sim$} & \multicolumn{2}{|c|}{$40 \sim$} \\
\hline & & M & $\mathrm{F}$ & M & $\mathbf{F}$ & M & $\mathrm{F}$ & M & $\mathrm{F}$ \\
\hline \multicolumn{2}{|c|}{ Number of Cases } & 61 & 39 & 165 & 109 & 119 & 94 & 153 & 82 \\
\hline \multirow{2}{*}{$\mathrm{V}_{1}$} & Mn., SD & $\begin{array}{c}0.020 \\
0.008\end{array}$ & $\begin{array}{c}0.019 \\
0.007\end{array}$ & $\begin{array}{c}0.020 \\
0.006\end{array}$ & $\begin{array}{c}0.019 \\
0.007\end{array}$ & $\begin{array}{c}0.022 \\
0.008\end{array}$ & $\begin{array}{r}0.020 \\
0.005\end{array}$ & $\begin{array}{c}0.020 \\
0.007\end{array}$ & $\begin{array}{c}0.020 \\
0.006\end{array}$ \\
\hline & Max. $\sim$ Min. & $0.054 \sim 0$ & $0.049 \sim 0$ & $\begin{array}{r}0.044 \sim \\
0.005\end{array}$ & $\begin{array}{r}0.049 \sim 00 \\
0.005\end{array}$ & $0.074 \sim 0$ & $\begin{array}{r}0.039 \sim \\
0.010\end{array}$ & $\begin{array}{r}0.054 \sim \\
0.005\end{array}$ & $\begin{array}{r}0.034 \sim \\
0.005\end{array}$ \\
\hline \multirow{2}{*}{$\mathbf{V}_{2}$} & Mn., SD & $\begin{array}{c}0.021 \\
0.006\end{array}$ & $\begin{array}{l}0.021 \\
\quad 0.007\end{array}$ & $\begin{array}{c}0.023 \\
0.006\end{array}$ & $\begin{array}{r}0.021 \\
0.006\end{array}$ & 0.024 & $\begin{array}{l}0.022 \\
0.006\end{array}$ & $\begin{array}{c}0.023 \\
0.007\end{array}$ & $\begin{array}{l}0.022 \\
0.005\end{array}$ \\
\hline & Max. $\sim$ Min & $0.044 \sim 0$ & $\begin{array}{r}0.049 \sim \\
0.010\end{array}$ & $\begin{array}{r}0.044 \sim \\
0.005\end{array}$ & $\begin{array}{r}0.049 \sim \\
0.005\end{array}$ & $\begin{array}{r}0.049 \sim \\
0.005\end{array}$ & $\begin{array}{r}0.044 \sim \\
0.005\end{array}$ & $\begin{array}{r}0.054 \sim \\
0.005\end{array}$ & $\begin{array}{r}0.034 \sim \\
0.005\end{array}$ \\
\hline \multirow{2}{*}{$\frac{V_{5}^{i}}{i}$} & Mn., SD. & $\begin{array}{c}0.035 \\
0.010\end{array}$ & $\begin{array}{c}0.030 \\
0.008\end{array}$ & $\begin{array}{r}0.035 \\
0.010\end{array}$ & $\begin{array}{c}0.029 \\
0.009\end{array}$ & $\begin{array}{l}0.036 \\
0.009\end{array}$ & $\begin{array}{r}0.030 \\
0.007\end{array}$ & $\begin{array}{c}0.034, \\
0.009\end{array}$ & $\begin{array}{c}0.032 \\
0.008\end{array}$ \\
\hline & Max. Min. & $0,064 \sim 0$ & $\begin{array}{r}0.049 \sim \\
0.010\end{array}$ & $\begin{array}{r}0.054 \sim \\
0.010\end{array}$ & $\begin{array}{r}0.054 \sim \\
0.010\end{array}$ & $0.064 \sim 0$ & $\begin{array}{r}0.044 \sim \\
0.010\end{array}$ & $\begin{array}{r}0.054 \sim \\
0.012\end{array}$ & $\begin{array}{r}0.054 \sim \\
0.010\end{array}$ \\
\hline \multirow{2}{*}{$\bar{v}_{6}$} & $\mathrm{Mn}_{\mathrm{n}}, \mathrm{SD}$ & $\begin{array}{c}0.036 \\
0.010\end{array}$ & $\begin{array}{c}0.029 \\
0.008\end{array}$ & $\begin{array}{c}0.037 \\
0.006\end{array}$ & $\begin{array}{c}0.030 \\
0.009\end{array}$ & $\begin{array}{l}0.037 \\
0.010\end{array}$ & $\begin{array}{l}0.030 \\
0.008\end{array}$ & $\begin{array}{c}0.036 \\
0.008\end{array}$ & $\begin{array}{r}0.033 \\
0.008\end{array}$ \\
\hline & Max,$\sim \operatorname{Min}$ & $\begin{array}{r}0.036 \sim \\
0.010\end{array}$ & $\begin{array}{r}0.044 \sim \\
0.010\end{array}$ & $\begin{array}{r}0.054 \sim \\
0.010\end{array}$ & $\begin{array}{l}0.054 \sim \\
0.010\end{array}$ & $0.054 \sim 0$ & $\begin{array}{r}0.049 \sim \\
0.015\end{array}$ & $\begin{array}{r}0.064 \sim \\
0.010\end{array}$ & $\begin{array}{r}0.044 \sim \\
0.010\end{array}$ \\
\hline
\end{tabular}

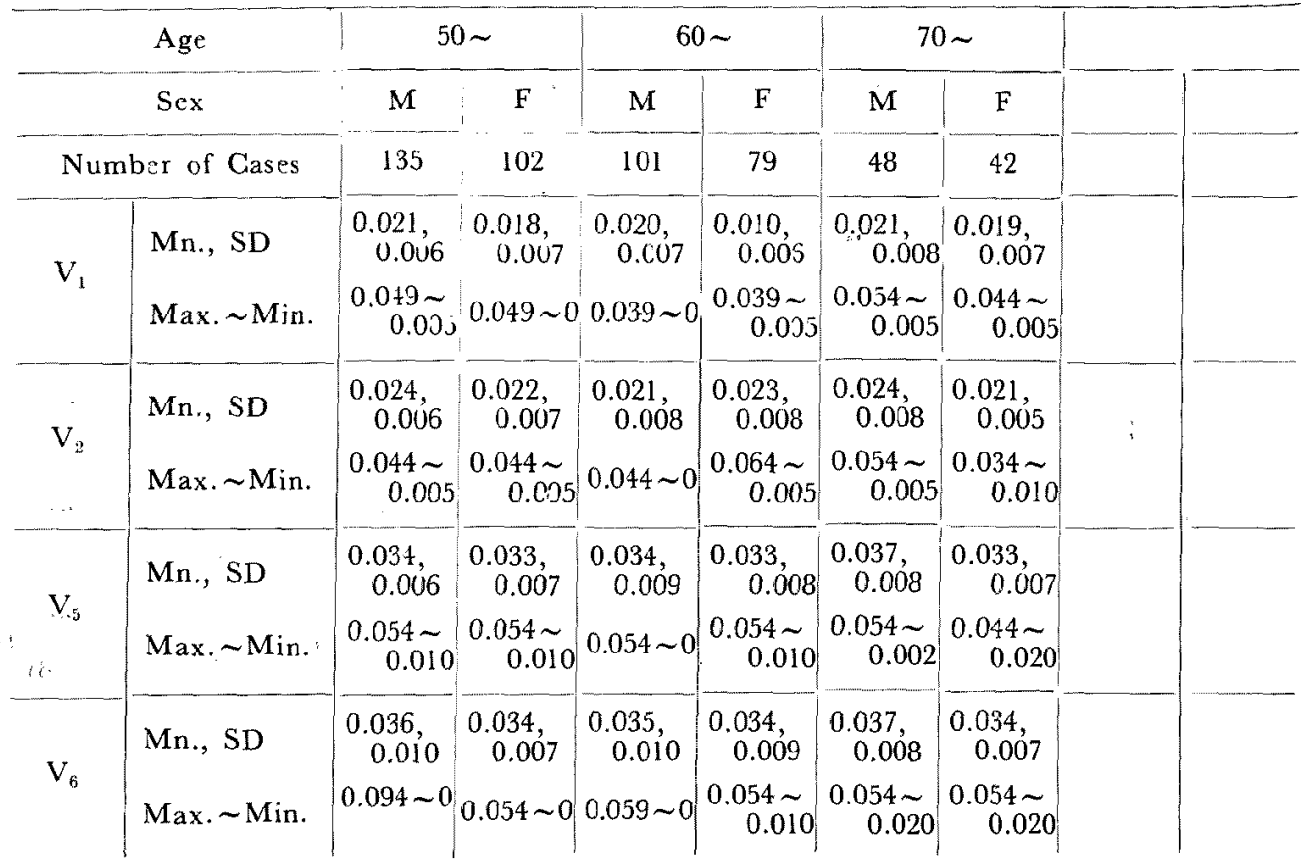




\section{$U$ Deflection (Table XIV)}

The mean values are positive and very small in all standard leads and unipolar extremity leads which do not exceed $0.2 \mathrm{~mm}$. Lead II shows the largest value among extremity leads. There are no remarkable differences with ages and sex.

In lead $V_{2}$ or $V_{3}$, the value takes the largest among the precordial lead. In the voltage of $U$, there are some tendencies to decrease with age, and to be larger in the male group.

\section{Ventricular Activation Time (VAT) (Table XV)}

$\mathrm{V}_{1}$ : The mean values and standard deviations are in a narrow range through the all age groups. The mean values range $0.022 \mathrm{sec}$. to $0.018 \mathrm{sec}$. and standard deviations indicate from $0.006 \mathrm{sec}$. to $0.008 \mathrm{sec}$. The upper limit of normal range is from $0.038 \mathrm{sec}$. to $0.031 \mathrm{sec}$. There are no remarkable differences with age and sex.

$\mathrm{V}_{2}$ : The various values have the same tendency as in lead $V_{1}$ except the increase of their values.

$V_{5}$ and $V_{6}$ : There are little differences between $V_{5}$ and $V_{6}$ in the values of VAT. In $V_{6}$, the mean values range from $0.37 \mathrm{sec}$. to $0.029 \mathrm{sec}$, and the upper limits are from $0.057 \mathrm{sec}$. to $0.045 \mathrm{sec}$. There are no remarkable differences with age, but the mean values of male groups are slightly larger than that of female groups.

\section{Discussion}

As recognizde by many authors, there are many variables which affect the normal values of electrocardiogram.

In this report, the effects of age and sex on electrocardiogram are mainly considered. The other variables to affect the electrocardiogram, are regarded to be equalized by relatively large samples.

As for age and sex, there are some differences in the voltages of some items of electrocardiogram. The voltage of $\mathrm{R}$ deflections in lead II, III, ${ }_{a} V_{F}$ and some of precordial leads decreases with age, and is larger in male groups than in female groups in the precordial leads. The same tendencies are observed in $\mathrm{S}$ deflection and also in $\mathrm{T}$ deflection. These results may suggest that when the diagnosis of ventricular hypertrophy is made from the deflections of electrocardiogram, age and sex should be considered carefully. 
Significance of displacement of S-T segment should be considered in each lead, because the lowest limit in lead $I$ is $-0.05 \mathrm{~mm}$., but in lead II or III, it exceeds $-0.05 \mathrm{~mm}$. and may reach to $-0.08 \mathrm{~mm}$. In precordial leads, it ranges more widely. It may be also important that there are some differences with sex in the S-T segment.

\section{Summary}

The survey on the normal values of electrocardiograms of the Japanese was conducted by Committee on the Criteria of Heart Diseases and Electrocardiogram. Electrocardiographic records from 1,329 Japanese people over 15 years of age, who were considered to be clinically normal, were analyzed according to age and sex. Among the various electrocardiographic component parts, 14 items were measured in the standard leads, unipolar extremity leads and precordial leads. The mean value, maximum value and minimum value were listed according to the items, age and sex. The standard deviation was calculated in some items. The normal range of electrocardiographic items and their difference with sex and age were discussed. 